\title{
The Sloan Digital Sky Survey Reverberation Mapping Project: $\mathrm{H} \alpha$ and $\mathrm{H} \beta$ Reverberation Measurements from First-year Spectroscopy and Photometry
}

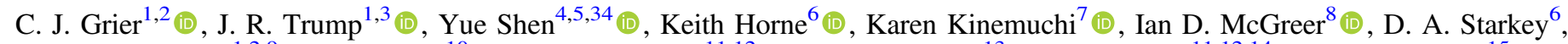
W. N. Brandt ${ }^{1,2,9}$ (1) P. B. Hall ${ }^{10}$ (1) , C. S. Kochanek ${ }^{11,12}$ (1) , Yuguang Chen $^{13}$, K. D. Denney ${ }^{11,12,14}$, Jenny E. Greene ${ }^{15}$, L. C. $\mathrm{Ho}^{16,17}$, , Y. Homayouni ${ }^{3}$, Jennifer I-Hsiu Li ${ }^{4}$, Liuyi $\mathrm{Pei}^{4}$, B. M. Peterson ${ }^{11,12,18}$ (D), P. Petitjean ${ }^{19}$, D. P. Schneider ${ }^{1,2}$,

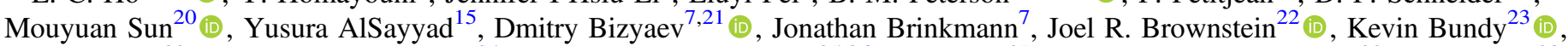
K S. Dawson ${ }^{22}$ (1), Sarah Eftekharzadeh ${ }^{24}$, J. G. Fernandez-Trincado ${ }^{25,26}$, Yang Gao ${ }^{27}$ (i), Timothy A. Hutchinson ${ }^{22}$ (1) , Siyao Jia ${ }^{28}$, Linhua Jiang ${ }^{16}$ (1), Daniel Oravetz ${ }^{7}$, Kaike Pan $^{7}$ (10), Isabelle Paris ${ }^{29}$, Kara A. Ponder ${ }^{30}$ (1) , Christina Peters $^{31}$, Jesse Rogerson ${ }^{32}$ (1), Audrey Simmons ${ }^{7}$ (1D, Robyn Smith ${ }^{33}$, and and Ran Wang ${ }^{16}$

${ }^{1}$ Dept. of Astronomy and Astrophysics, The Pennsylvania State University, 525 Davey Laboratory, University Park, PA 16802, USA

${ }^{2}$ Institute for Gravitation and the Cosmos, The Pennsylvania State University, University Park, PA 16802, USA

${ }^{3}$ Department of Physics, University of Connecticut, 2152 Hillside Road, Unit 3046, Storrs, CT 06269, USA

${ }^{4}$ Department of Astronomy, University of Illinois at Urbana-Champaign, Urbana, IL 61801, USA

${ }^{5}$ National Center for Supercomputing Applications, University of Illinois at Urbana-Champaign, Urbana, IL 61801, USA

${ }^{6}$ SUPA Physics and Astronomy, University of St Andrews, Fife, KY16 9SS, Scotland, UK

${ }^{7}$ Apache Point Observatory and New Mexico State University, P.O. Box 59, Sunspot, NM, 88349-0059, USA

${ }^{8}$ Steward Observatory, The University of Arizona, 933 North Cherry Avenue, Tucson, AZ 85721, USA

${ }^{9}$ Department of Physics, 104 Davey Lab, The Pennsylvania State University, University Park, PA 16802, USA

${ }^{10}$ Department of Physics and Astronomy, York University, Toronto, ON M3J 1P3, Canada

${ }^{11}$ Department of Astronomy, The Ohio State University, 140 West 18th Avenue, Columbus, OH 43210, USA

${ }^{12}$ Center for Cosmology and AstroParticle Physics, The Ohio State University, 191 West Woodruff Avenue, Columbus, OH 43210, USA

${ }^{13}$ Cahill Center for Astronomy and Astrophysics, California Institute of Technology, 1200 East California Boulevard, MC 249-17, CA 91125, USA

${ }^{14}$ Illumination Works, LLC, 5550 Blazer Parkway, Dublin, OH, 43017, USA

${ }^{15}$ Department of Astrophysical Sciences, Princeton University, Princeton, NJ 08544, USA

${ }^{16}$ Kavli Institute for Astronomy and Astrophysics, Peking University, Beijing 100871, China

${ }_{18}^{17}$ Department of Astronomy, School of Physics, Peking University, Beijing 100871, China

${ }^{18}$ Space Telescope Science Institute, 3700 San Martin Drive, Baltimore, MD 21218, USA

${ }^{19}$ Institut d'Astrophysique de Paris, Université Paris 6-CNRS, UMR7095, 98bis Boulevard Arago, F-75014 Paris, France

${ }^{20}$ Key Laboratory for Research in Galaxies and Cosmology, Center for Astrophysics, Department of Astronomy,

University of Science and Technology of China, Chinese Academy of Sciences, Hefei, Anhui 230026, China

${ }^{21}$ Sternberg Astronomical Institute, Moscow State University, Moscow, Russia

22 Department of Physics and Astronomy, University of Utah, 115 South 1400 East, Salt Lake City, UT 84112, USA

${ }^{23}$ UCO/Lick Observatory, University of California, Santa Cruz, 1156 High St., Santa Cruz, CA 95064, USA

${ }^{24}$ Department of Physics and Astronomy, University of Wyoming, Laramie, WY 82071, USA

${ }^{25}$ Departamento de Astronomía, Casilla 160-C, Universidad de Concepción, Concepción, Chile

${ }^{26}$ Institut Utinam, CNRS UMR6213, Univ. Bourgogne Franche-Comté, OSU THETA, Observatoire de Besançon, BP 1615, 25010 Besançon Cedex, France

${ }^{27}$ Department of Engineering Physics and Center for Astrophysics, Tsinghua University, Beijing 100084, China; Key Laboratory of Particle and Radiation Imaging (Tsinghua University), Ministry of Education, Beijing 100084, China

${ }^{28}$ Department of Astronomy, University of California, Berkeley, CA 94720, USA

${ }^{29}$ Aix-Marseille Université, CNRS, LAM (Laboratoire d'Astrophysique de Marseille) UMR 7326, F-13388, Marseille, France

${ }^{30}$ Pittsburgh Particle Physics, Astrophysics, and Cosmology Center (PITT PACC), Physics and Astronomy Department, University of Pittsburgh, Pittsburgh, PA 15260, USA

${ }^{31}$ Dunlap Institute \& Department of Astronomy and Astrophysics, University of Toronto, 50 St George Street, Toronto, ON M5S 3H4, Canada

${ }^{32}$ Canada Aviation and Space Museum, 11 Aviation Parkway, Ottawa, ON, K1K 4Y5, Canada

${ }^{33}$ Department of Astronomy, University of Maryland, Stadium Drive, College Park, MD 20742-2421, USA

Received 2017 April 25; revised 2017 October 3; accepted 2017 October 14; published 2017 December 7

\begin{abstract}
We present reverberation mapping results from the first year of combined spectroscopic and photometric observations of the Sloan Digital Sky Survey Reverberation Mapping Project. We successfully recover reverberation time delays between the $g+i$ band emission and the broad $\mathrm{H} \beta$ emission line for a total of 44 quasars, and for the broad $\mathrm{H} \alpha$ emission line in 18 quasars. Time delays are computed using the JAVELIN and CREAM software and the traditional interpolated cross-correlation function (ICCF): using well-defined criteria, we report measurements of $32 \mathrm{H} \beta$ and $13 \mathrm{H} \alpha$ lags with JAVELIN, $42 \mathrm{H} \beta$ and $17 \mathrm{H} \alpha$ lags with CREAM, and $16 \mathrm{H} \beta$ and eight $\mathrm{H} \alpha$ lags with the ICCF. Lag values are generally consistent among the three methods, though we typically measure smaller uncertainties with JAVELIN and CREAM than with the ICCF, given the more physically motivated light curve interpolation and more robust statistical modeling of the former two methods. The median redshift of our $\mathrm{H} \beta$-detected sample of quasars is 0.53 , significantly higher than that of the previous reverberation mapping sample. We find that in most objects, the time delay of the $\mathrm{H} \alpha$ emission is consistent with or slightly longer than that of $\mathrm{H} \beta$. We measure black hole masses using our measured time delays and line widths for these quasars. These black hole mass measurements are mostly consistent with expectations based on the local $M_{\mathrm{BH}}-\sigma_{*}$ relationship, and are also consistent with single-epoch black hole mass measurements. This work increases the current sample size of reverberation-mapped active galaxies by about two-thirds and represents the first large sample of reverberation mapping observations beyond the local universe $(z<0.3)$.
\end{abstract}

\footnotetext{
${ }^{34}$ Alfred P. Sloan Research Fellow.
} 
Key words: galaxies: active - galaxies: nuclei - quasars: emission lines - quasars: general

Supporting material: figure sets, machine-readable tables

\section{Introduction}

Over the past few decades, the technique of reverberation mapping (RM; e.g., Blandford \& McKee 1982; Peterson et al. 2004) has emerged as a powerful tool for measuring black hole masses $\left(M_{\mathrm{BH}}\right)$ in active galactic nuclei (AGNs). RM allows a measurement of the size of the broad-line-emitting region (BLR), which is photoionized by continuum emission from closer to the black hole $(\mathrm{BH})$. Variability of the continuum is echoed by the BLR after a time delay that corresponds to the light travel time between the continuumemitting region and the BLR; this time delay provides a measurement of the distance between the two regions and thus a characteristic size for the BLR $\left(R_{\mathrm{BLR}}\right)$.

Assuming that the motion of the BLR gas is dominated by the gravitational field of the central $\mathrm{BH}$, we can combine $R_{\mathrm{BLR}}$ with the broad-emission-line width $(\Delta V)$ to measure a $\mathrm{BH}$ mass of

$$
M_{\mathrm{BH}}=\frac{f R_{\mathrm{BLR}} \Delta V^{2}}{G},
$$

where the dimensionless scale factor $f$ accounts for the orientation, kinematics, and structure of the BLR.

Thus far, about 60 AGNs have $M_{\mathrm{BH}}$ measurements obtained through reverberation mapping (e.g., Kaspi et al. 2000, 2005; Peterson et al. 2004; Bentz et al. 2009, 2010; Denney et al. 2010; Grier et al. 2012; Du et al. 2014, 2016a, 2016b; Barth et al. 2015; Hu et al. 2015). Bentz \& Katz (2015) provide a running compilation of these measurements. ${ }^{35}$ Due to the stringent observational requirements of RM measurements, the existing sample is mainly composed of nearby $(z<0.3)$, lower-luminosity AGNs that have sufficiently short time delays to be measurable with a few months of monitoring using a modest-sized telescope. Because they are low redshift, these studies typically focus on the $\mathrm{H} \beta$ emission line and other nearby lines in the observed-frame optical.

RM measurements have established the radius-luminosity $(R-L)$ relationship (e.g., Kaspi et al. 2007; Bentz et al. 2013), which allows one to estimate the BLR size with a single spectrum and thus estimate $M_{\mathrm{BH}}$ for large numbers of quasars at greater distances where traditional RM campaigns are impractical (e.g., Shen et al. 2011). However, the current RM sample may be biased; beyond the fact that these AGNs are low redshift, they do not span the full range of AGN emission-line properties (see Figure 1 of Shen et al. 2015a). In addition, the $R-L$ relation is only well calibrated for $\mathrm{H} \beta$, but most higherredshift, single-epoch $M_{\mathrm{BH}}$ estimates are made using $\mathrm{C}$ IV or $\mathrm{Mg}$ II. There are only a handful of RM measurements for C IV, particularly at high redshift (e.g., Kaspi et al. 2007), and only a few reliable $\mathrm{Mg}$ II lag measurements have been reported (Metzroth et al. 2006; Shen et al. 2016b). Such measurements are difficult to make because higher-luminosity quasars have longer time delays and larger time dilation factors and thus require observations spanning years rather than months.

The Sloan Digital Sky Survey Reverberation Mapping Project (SDSS-RM) is a dedicated multiobject RM program that began in

\footnotetext{
35 http://www.astro.gsu.edu/AGNmass/
}

2014 (see Shen et al. 2015a for details). The major goals of this program are to expand the number of reverberation-mapped AGNs, the range of AGN parameters spanned by the RM sample, and the redshift and luminosity range of the RM sample, and to firmly establish $R-L$ relationships for C IV and $\mathrm{Mg}$ II. SDSS-RM started as an ancillary program of the SDSS-III survey (Eisenstein et al. 2011) on the SDSS $2.5 \mathrm{~m}$ telescope (Gunn et al. 2006), monitoring 849 quasars in a single field with the Baryon Oscillation Spectroscopic Survey (BOSS) spectrograph (Dawson et al. 2013; Smee et al. 2013). Additional photometric data were acquired with the $3.6 \mathrm{~m}$ Canada-France-Hawaii Telescope (CFHT) and the Steward Observatory $2.3 \mathrm{~m}$ Bok telescope to improve the cadence of the continuum light curves. Observations for the program have continued in 2015-2017 as part of SDSS-IV (Blanton et al. 2017) to extend the temporal baseline of the program.

While the primary goals of this program are to obtain RM measurements for $\gtrsim 100$ quasars, we have been pursuing a wide variety of ancillary science goals as well, ranging from studies of emission-line and host-galaxy properties to the variability of broad absorption lines (Grier et al. 2015; Matsuoka et al. 2015; Shen et al. 2015b, 2016a; Sun et al. 2015; Denney et al. 2016). The first RM results from this program were reported by Shen et al. (2016b), who measured emission-line lags in the $\mathrm{H} \beta$ and $\mathrm{Mg}$ II emission lines in 15 of the brightest, relatively lowredshift sources in our sample using the first year of SDSS-RM spectroscopy alone (i.e., no photometric data were used). Li et al. (2017) also measured composite RM lags using a lowluminosity subset and the first year of spectroscopy.

We here report results based on the combined spectroscopic and imaging data from the first year of observations, focusing on the $\mathrm{H} \beta$ and $\mathrm{H} \alpha$ emission lines in the low-redshift $(z<1.1)$ subset of the SDSS-RM sample. We detect significant lags in about $20 \%$ of our sample. In Section 2, we describe the sample of quasars in our study, present details of the data, and discuss data preparation. We discuss our time-series analysis methods in Section 3 and our results in Section 4, and we summarize our findings in Section 5. Throughout this work, we adopt a $\Lambda$ CDM cosmology with $\Omega_{\Lambda}=0.7, \Omega_{M}=0.3$, and $h=0.7$.

\section{Data and Data Processing}

\subsection{The Quasar Sample}

We selected our objects from the full SDSS-RM quasar sample, which is flux-limited $(i<21.7$; measurements by Ahn et al. 2014) and contains 849 quasars with redshifts of $0.1<z<4.5$. A complete description of the parent sample and the properties of the quasars will be reported by Y. Shen et al. (2017, in preparation). Within the full sample, there are 222 quasars in the $0.11<z<1.13$ redshift range that places $\mathrm{H} \beta$ in the wavelength range of the SDSS spectra. Basic information on these quasars is given in Table 1, including several spectral measurements made by Shen et al. (2015b). Figure 1 presents the distributions of the quasars in redshift, magnitude, typical spectral signal-to-noise ratio $(\mathrm{S} / \mathrm{N})$, and luminosity. Of the 222 quasars, 55 are at low-enough redshifts $(z<0.6)$ for $\mathrm{H} \alpha$ to fall within the observed wavelength range of the spectra as well. 
Table 1

Quasar Sample Information

\begin{tabular}{|c|c|c|c|c|c|c|c|c|c|}
\hline RMID & $\begin{array}{c}\text { SDSS } \\
\text { Identifier }\end{array}$ & $\begin{array}{l}\text { R.A. }^{\text {a }} \\
\text { (deg) } \\
\text { (J2000) }\end{array}$ & $\begin{array}{c}\text { Decl. }^{\text {a }} \\
\text { (deg) } \\
(\mathrm{J} 2000)\end{array}$ & $z^{\mathrm{a}}$ & $i \mathrm{mag}^{\mathrm{a}}$ & $\begin{array}{c}\mathrm{MED}^{\mathrm{b}} \\
\mathrm{S} / \mathrm{N}\end{array}$ & $\begin{array}{c}\mathrm{AGN} \\
\log \lambda L_{\lambda 5100} \mathrm{c} \\
\left(\mathrm{erg} \mathrm{s}^{-1}\right)\end{array}$ & $\begin{array}{c}\text { Host } \\
\log \lambda L_{\lambda 5100} \mathrm{c} \\
\left(\mathrm{erg} \mathrm{s}^{-1}\right)\end{array}$ & $\begin{array}{c}\log M_{\mathrm{BH}, \mathrm{SE}}{ }^{\mathrm{c}} \\
\quad\left(M_{\odot}\right)\end{array}$ \\
\hline 005 & $\mathrm{~J} 141541.41+530424.3$ & 213.9225 & 53.0734 & 1.020 & 20.716 & 5.0 & 44.5 & & 8.1 \\
\hline 009 & $\mathrm{~J} 141359.51+531049.3$ & 213.4980 & 53.1804 & 0.898 & 20.473 & 4.3 & 44.1 & 43.8 & 7.9 \\
\hline 016 & J141606.95+530929.8 & 214.0290 & 53.1583 & 0.848 & 19.716 & 11.5 & 44.8 & 43.9 & 9.0 \\
\hline 017 & $\mathrm{~J} 141324.28+530527.0$ & 213.3511 & 53.0908 & 0.456 & 19.213 & 14.1 & 43.9 & 44.2 & 8.4 \\
\hline 018 & $\mathrm{~J} 141323.27+531034.3$ & 213.3469 & 53.1762 & 0.849 & 20.205 & 5.2 & 44.3 & 44.2 & 8.9 \\
\hline 020 & $\mathrm{~J} 141411.66+525149.0$ & 213.5486 & 52.8636 & 1.124 & 21.529 & 2.0 & $\ldots$ & $\ldots$ & $\ldots$ \\
\hline 021 & $\mathrm{~J} 141314.97+530139.4$ & 213.3124 & 53.0276 & 1.026 & 21.167 & 3.0 & 44.5 & 44.0 & 7.6 \\
\hline 027 & $\mathrm{~J} 141600.80+525255.5$ & 214.0033 & 52.8821 & 1.023 & 20.968 & 2.1 & 44.4 & 44.2 & 8.5 \\
\hline 029 & $\mathrm{~J} 141310.71+525750.2$ & 213.2946 & 52.9640 & 0.816 & 21.142 & 2.6 & 43.8 & 43.7 & 7.7 \\
\hline 033 & $\mathrm{~J} 141532.36+524905.9$ & 213.8848 & 52.8183 & 0.715 & 20.490 & 6.6 & 44.1 & 43.9 & 7.6 \\
\hline 040 & $\mathrm{~J} 141648.89+530903.6$ & 214.2037 & 53.1510 & 0.600 & 20.868 & 3.4 & 43.5 & 43.5 & 7.1 \\
\hline 050 & $\mathrm{~J} 141522.54+524421.5$ & 213.8439 & 52.7393 & 0.526 & 20.818 & 4.3 & 43.6 & 43.7 & 8.2 \\
\hline 053 & $\mathrm{~J} 141222.76+530648.6$ & 213.0948 & 53.1135 & 0.894 & 21.361 & 2.8 & 44.0 & 44.0 & 8.0 \\
\hline 061 & $\mathrm{~J} 141559.99+524416.1$ & 214.0000 & 52.7378 & 0.983 & 21.379 & 3.3 & 44.4 & 44.2 & 8.1 \\
\hline 062 & J141417.69+532810.8 & 213.5737 & 53.4697 & 0.808 & 20.528 & 4.2 & 44.0 & 44.0 & 8.6 \\
\hline 077 & $\mathrm{~J} 141747.02+530349.7$ & 214.4459 & 53.0638 & 0.914 & 21.124 & 2.7 & 44.0 & 43.8 & 7.8 \\
\hline 078 & $\mathrm{~J} 141154.17+531119.5$ & 212.9757 & 53.1887 & 0.581 & 20.134 & 13.3 & 44.4 & $\ldots$ & 8.8 \\
\hline 085 & $\mathrm{~J} 141539.59+523727.9$ & 213.9150 & 52.6244 & 0.237 & 18.563 & 18.5 & 43.3 & 43.5 & 8.1 \\
\hline 088 & $\mathrm{~J} 141151.78+525344.1$ & 212.9657 & 52.8956 & 0.516 & 19.731 & 10.9 & 44.1 & 43.7 & 8.5 \\
\hline 090 & $\mathrm{~J} 141144.12+531508.6$ & 212.9338 & 53.2524 & 0.923 & 20.753 & 1.3 & 43.8 & 44.4 & 8.8 \\
\hline 101 & $\mathrm{~J} 141214.20+532546.7$ & 213.0592 & 53.4296 & 0.458 & 18.837 & 21.3 & 44.4 & 43.4 & 7.9 \\
\hline 102 & $\mathrm{~J} 141352.99+523444.2$ & 213.4708 & 52.5790 & 0.860 & 19.536 & 14.4 & 44.7 & $\ldots$ & 8.0 \\
\hline 103 & $\mathrm{~J} 141155.26+524733.6$ & 212.9802 & 52.7927 & 0.517 & 19.928 & 4.8 & 43.7 & 44.1 & 9.2 \\
\hline 111 & $\mathrm{~J} 141626.48+533406.5$ & 214.1104 & 53.5685 & 1.133 & 20.524 & 3.4 & $\ldots$ & $\ldots$ & $\ldots$ \\
\hline 118 & $\mathrm{~J} 141412.78+523209.0$ & 213.5533 & 52.5358 & 0.714 & 19.318 & 21.2 & 44.8 & $\ldots$ & 8.3 \\
\hline 121 & $\mathrm{~J} 141125.70+524924.2$ & 212.8571 & 52.8234 & 0.968 & 21.300 & 3.3 & 44.3 & 43.8 & 8.0 \\
\hline 122 & $\mathrm{~J} 141628.70+523346.4$ & 214.1196 & 52.5629 & 0.986 & 20.933 & 5.0 & 44.6 & 44.4 & 8.1 \\
\hline 123 & $\mathrm{~J} 141837.85+531017.6$ & 214.6577 & 53.1716 & 0.889 & 20.440 & 6.7 & 44.5 & $\ldots$ & 8.6 \\
\hline 125 & $\mathrm{~J} 141149.92+532721.1$ & 212.9580 & 53.4559 & 1.076 & 21.524 & 2.4 & $\ldots$ & $\ldots$ & $\ldots$ \\
\hline 126 & $\mathrm{~J} 141408.76+533938.3$ & 213.5365 & 53.6606 & 0.192 & 18.561 & 20.7 & 43.3 & 43.5 & 7.3 \\
\hline 133 & $\mathrm{~J} 141731.59+533224.4$ & 214.3816 & 53.5401 & 0.981 & 20.531 & 5.7 & 44.4 & 44.0 & 7.8 \\
\hline 134 & $\mathrm{~J} 141054.58+531532.9$ & 212.7274 & 53.2591 & 0.964 & 19.825 & 11.1 & 44.8 & 44.1 & 8.3 \\
\hline 140 & $\mathrm{~J} 141856.21+531007.1$ & 214.7342 & 53.1687 & 0.609 & 20.162 & 5.3 & 43.8 & 44.0 & 7.5 \\
\hline 141 & $\mathrm{~J} 141324.66+522938.2$ & 213.3527 & 52.4939 & 0.812 & 20.551 & 3.9 & 44.2 & 43.9 & 8.7 \\
\hline 160 & $\mathrm{~J} 141041.25+531849.0$ & 212.6719 & 53.3136 & 0.359 & 19.679 & 9.0 & 43.8 & $\ldots$ & 8.2 \\
\hline 165 & $\mathrm{~J} 141804.59+523745.0$ & 214.5191 & 52.6292 & 1.086 & 21.175 & 3.5 & $\ldots$ & $\ldots$ & $\ldots$ \\
\hline 168 & $\mathrm{~J} 141723.39+523153.9$ & 214.3474 & 52.5316 & 0.484 & 21.137 & 2.4 & 43.0 & 43.5 & 7.2 \\
\hline 171 & $\mathrm{~J} 141321.13+534344.7$ & 213.3380 & 53.7291 & 0.790 & 20.992 & 3.4 & 44.0 & 43.7 & 7.5 \\
\hline 173 & $\mathrm{~J} 141147.60+523414.6$ & 212.9483 & 52.5707 & 0.970 & 19.917 & 10.1 & 44.8 & 44.4 & 9.0 \\
\hline 175 & $\mathrm{~J} 141531.32+522407.8$ & 213.8805 & 52.4022 & 0.819 & 21.301 & 2.9 & 44.0 & 43.9 & 7.9 \\
\hline 177 & $\mathrm{~J} 141724.59+523024.9$ & 214.3525 & 52.5069 & 0.482 & 19.560 & 10.8 & 44.0 & 43.8 & 8.4 \\
\hline 184 & $\mathrm{~J} 141721.80+534102.6$ & 214.3408 & 53.6841 & 0.193 & 17.857 & 30.0 & 43.7 & 43.4 & 7.2 \\
\hline 185 & $\mathrm{~J} 141735.95+523029.9$ & 214.3998 & 52.5083 & 0.987 & 19.889 & 8.1 & 44.8 & $\ldots$ & 8.9 \\
\hline 187 & $\mathrm{~J} 141005.21+531003.9$ & 212.5217 & 53.1677 & 0.997 & 21.119 & 1.2 & 43.9 & 44.4 & 9.1 \\
\hline 191 & $\mathrm{~J} 141645.58+534446.8$ & 214.1899 & 53.7463 & 0.442 & 20.448 & 6.2 & 43.6 & 43.6 & 7.5 \\
\hline 192 & $\mathrm{~J} 141649.44+522531.0$ & 214.2060 & 52.4253 & 1.024 & 19.971 & 6.8 & 45.0 & $\ldots$ & 8.5 \\
\hline 193 & $\mathrm{~J} 141542.16+522207.0$ & 213.9257 & 52.3686 & 1.003 & 20.498 & 7.2 & 44.8 & 44.0 & 7.7 \\
\hline 203 & $\mathrm{~J} 141811.34+533808.6$ & 214.5473 & 53.6357 & 0.977 & 20.583 & 5.3 & 44.4 & $\ldots$ & 8.2 \\
\hline 204 & $\mathrm{~J} 141221.73+522556.6$ & 213.0906 & 52.4324 & 0.922 & 18.575 & 20.6 & 45.1 & $\ldots$ & 8.7 \\
\hline 211 & $\mathrm{~J} 141522.01+535033.5$ & 213.8417 & 53.8426 & 0.971 & 19.448 & 8.9 & 44.7 & 44.3 & 8.2 \\
\hline 215 & $\mathrm{~J} 141952.23+531340.9$ & 214.9676 & 53.2280 & 0.884 & 21.290 & 3.6 & 44.2 & $\ldots$ & 8.7 \\
\hline 229 & $\mathrm{~J} 141018.04+532937.5$ & 212.5752 & 53.4937 & 0.470 & 20.271 & 4.7 & 43.6 & 43.5 & 8.0 \\
\hline 232 & $\mathrm{~J} 141651.26+522046.1$ & 214.2136 & 52.3461 & 0.807 & 20.776 & 3.8 & 44.0 & 44.1 & 7.6 \\
\hline 235 & $\mathrm{~J} 141111.30+534029.4$ & 212.7971 & 53.6748 & 0.785 & 19.872 & 10.0 & 44.4 & 43.9 & 8.4 \\
\hline 240 & $\mathrm{~J} 141420.87+521629.9$ & 213.5870 & 52.2750 & 0.762 & 20.879 & 3.6 & 43.9 & 44.3 & 8.5 \\
\hline 243 & $\mathrm{~J} 140924.89+530002.7$ & 212.3537 & 53.0007 & 0.659 & 20.036 & 8.3 & 44.3 & 43.6 & 8.5 \\
\hline 252 & $\mathrm{~J} 141751.14+522311.1$ & 214.4631 & 52.3864 & 0.281 & 19.768 & 7.1 & 42.7 & 43.5 & 8.6 \\
\hline 255 & $\mathrm{~J} 141525.41+535508.2$ & 213.8559 & 53.9189 & 0.992 & 21.471 & 2.0 & 44.2 & 44.4 & 8.2 \\
\hline 258 & $\mathrm{~J} 142027.51+530454.5$ & 215.1146 & 53.0818 & 0.994 & 20.762 & 2.3 & 44.4 & 43.9 & 8.5 \\
\hline 260 & $\mathrm{~J} 141018.04+523446.1$ & 212.5752 & 52.5795 & 0.995 & 21.636 & 15.5 & 45.0 & 43.7 & 8.1 \\
\hline 265 & $\mathrm{~J} 142023.88+531605.1$ & 215.0995 & 53.2681 & 0.734 & 20.645 & 6.8 & 44.2 & 44.0 & 8.3 \\
\hline 267 & J141112.72+534507.1 & 212.8030 & 53.7520 & 0.587 & 19.623 & 10.5 & 44.1 & 43.9 & 7.9 \\
\hline
\end{tabular}


Table 1

(Continued)

\begin{tabular}{|c|c|c|c|c|c|c|c|c|c|}
\hline RMID & $\begin{array}{c}\text { SDSS } \\
\text { Identifier }\end{array}$ & $\begin{array}{l}\text { R.A. }^{\text {a }} \\
(\mathrm{deg}) \\
(\mathrm{J} 2000)\end{array}$ & $\begin{array}{c}\text { Decl. }^{\mathrm{a}} \\
(\mathrm{deg}) \\
(\mathrm{J} 2000)\end{array}$ & $z^{\mathrm{a}}$ & $i \mathrm{mag}^{\mathrm{a}}$ & $\begin{array}{l}\mathrm{MED}^{\mathrm{b}} \\
\mathrm{S} / \mathrm{N}\end{array}$ & $\begin{array}{c}\text { AGN } \\
\log \lambda L_{\lambda 5100} \\
\left(\operatorname{erg~s}^{-1}\right)\end{array}$ & $\begin{array}{c}\text { Host } \\
\log \lambda L_{\lambda 5100} \mathrm{c} \\
\left(\operatorname{erg~s}^{-1}\right)\end{array}$ & $\begin{array}{c}\log M_{\mathrm{BH}, \mathrm{SE}}{ }^{\mathrm{c}} \\
\left(M_{\odot}\right)\end{array}$ \\
\hline 268 & $\mathrm{~J} 141043.36+534111.8$ & 212.6807 & 53.6866 & 0.650 & 20.718 & 3.9 & 43.7 & 44.0 & 8.5 \\
\hline 270 & $\mathrm{~J} 140943.01+524153.1$ & 212.4292 & 52.6981 & 0.421 & 20.095 & 3.7 & 43.5 & 43.6 & 8.3 \\
\hline 272 & $\mathrm{~J} 141625.71+535438.5$ & 214.1071 & 53.9107 & 0.263 & 18.822 & 23.2 & 43.9 & $\ldots$ & 7.8 \\
\hline 274 & $\mathrm{~J} 141949.82+533033.5$ & 214.9576 & 53.5093 & 0.793 & 20.546 & 4.9 & 44.3 & 44.0 & 8.3 \\
\hline 277 & $\mathrm{~J} 141409.44+535648.2$ & 213.5393 & 53.9467 & 0.825 & 20.649 & 3.8 & 44.0 & 44.1 & 7.8 \\
\hline 278 & $\mathrm{~J} 141717.07+521751.5$ & 214.3211 & 52.2976 & 1.022 & 20.587 & 5.4 & 44.8 & $\ldots$ & 8.5 \\
\hline 285 & $\mathrm{~J} 141650.92+521528.6$ & 214.2122 & 52.2579 & 1.034 & 21.300 & 3.3 & $\ldots$ & $\ldots$ & $\ldots$ \\
\hline 290 & $\mathrm{~J} 141138.06+534957.7$ & 212.9086 & 53.8327 & 1.078 & 19.865 & 7.5 & $\ldots$ & $\ldots$ & $\ldots$ \\
\hline 291 & J141643.24+521435.8 & 214.1802 & 52.2433 & 0.531 & 19.825 & 4.4 & 43.9 & 43.3 & 8.6 \\
\hline 296 & $\mathrm{~J} 141838.35+522359.3$ & 214.6598 & 52.3998 & 1.120 & 18.750 & 22.6 & $\ldots$ & $\ldots$ & $\ldots$ \\
\hline 297 & $\mathrm{~J} 141002.21+533730.2$ & 212.5092 & 53.6251 & 1.026 & 20.566 & 3.8 & 44.5 & 43.6 & 7.9 \\
\hline 300 & $\mathrm{~J} 141941.11+533649.6$ & 214.9213 & 53.6138 & 0.646 & 19.491 & 16.5 & 44.5 & 44.0 & 8.2 \\
\hline 301 & $\mathrm{~J} 142010.25+524029.6$ & 215.0427 & 52.6749 & 0.548 & 19.764 & 8.3 & 44.1 & 43.7 & 8.5 \\
\hline 302 & $\mathrm{~J} 140850.91+525750.9$ & 212.2121 & 52.9642 & 0.981 & 20.954 & 3.1 & 44.3 & 44.1 & 8.3 \\
\hline 303 & $\mathrm{~J} 141830.20+522212.5$ & 214.6259 & 52.3701 & 0.820 & 20.882 & 4.0 & 44.0 & 44.0 & 8.3 \\
\hline 305 & $\mathrm{~J} 141004.27+523141.0$ & 212.5178 & 52.5281 & 0.527 & 19.505 & 11.3 & 44.2 & 43.7 & 7.9 \\
\hline 306 & $\mathrm{~J} 141622.95+521212.2$ & 214.0956 & 52.2034 & 1.123 & 20.389 & 3.2 & $\ldots$ & $\ldots$ & $\ldots$ \\
\hline 308 & $\mathrm{~J} 141302.60+535729.9$ & 213.2608 & 53.9583 & 1.130 & 20.815 & 2.7 & $\ldots$ & $\ldots$ & $\ldots$ \\
\hline 316 & $\mathrm{~J} 142052.44+525622.4$ & 215.2185 & 52.9396 & 0.676 & 18.028 & 34.0 & 45.0 & $\ldots$ & 8.5 \\
\hline 320 & $\mathrm{~J} 142038.52+532416.5$ & 215.1605 & 53.4046 & 0.265 & 19.467 & 14.4 & 43.4 & 43.4 & 8.1 \\
\hline 323 & $\mathrm{~J} 141123.22+535204.2$ & 212.8467 & 53.8678 & 0.804 & 21.104 & 2.5 & 43.6 & 44.1 & 7.8 \\
\hline 324 & $\mathrm{~J} 141658.28+521205.1$ & 214.2428 & 52.2014 & 0.602 & 19.857 & 13.2 & 44.3 & 43.8 & 8.8 \\
\hline 328 & $\mathrm{~J} 141313.27+535944.0$ & 213.3053 & 53.9956 & 1.076 & 19.897 & 13.5 & $\ldots$ & $\ldots$ & $\ldots$ \\
\hline 329 & $\mathrm{~J} 141659.76+535806.7$ & 214.2490 & 53.9685 & 0.720 & 18.107 & 34.8 & 45.2 & $\ldots$ & 8.2 \\
\hline 331 & $\mathrm{~J} 142107.76+530318.2$ & 215.2823 & 53.0551 & 0.735 & 21.332 & 2.8 & 43.9 & 43.8 & 8.6 \\
\hline 333 & $\mathrm{~J} 141633.35+521001.1$ & 214.1389 & 52.1670 & 1.089 & 20.811 & 3.8 & $\ldots$ & $\ldots$ & $\ldots$ \\
\hline 336 & $\mathrm{~J} 141514.15+540222.9$ & 213.8089 & 54.0397 & 0.849 & 20.770 & 4.3 & 44.3 & 43.9 & 8.6 \\
\hline 337 & $\mathrm{~J} 142103.30+531822.4$ & 215.2638 & 53.3062 & 0.708 & 20.899 & 2.7 & 43.6 & 44.0 & 8.4 \\
\hline 338 & $\mathrm{~J} 141955.62+534007.2$ & 214.9818 & 53.6687 & 0.418 & 20.084 & 5.6 & 43.4 & 43.6 & 8.4 \\
\hline 341 & $\mathrm{~J} 141500.38+520658.6$ & 213.7516 & 52.1163 & 0.424 & 18.562 & 24.8 & 44.4 & $\ldots$ & 8.2 \\
\hline 350 & $\mathrm{~J} 141914.50+534810.6$ & 214.8104 & 53.8029 & 0.860 & 21.235 & 2.4 & 43.9 & 43.9 & 7.8 \\
\hline 354 & $\mathrm{~J} 141957.27+534157.9$ & 214.9887 & 53.6994 & 1.111 & 21.306 & 1.9 & $\ldots$ & $\ldots$ & $\ldots$ \\
\hline 355 & $\mathrm{~J} 141712.97+520957.5$ & 214.3040 & 52.1660 & 0.753 & 20.945 & 2.8 & 43.5 & 44.0 & 8.3 \\
\hline 356 & $\mathrm{~J} 141533.89+520558.0$ & 213.8912 & 52.0995 & 0.986 & 18.724 & 30.5 & 45.3 & $\ldots$ & 8.5 \\
\hline 369 & $\mathrm{~J} 141304.34+520659.3$ & 213.2681 & 52.1165 & 0.719 & 20.252 & 6.0 & 44.1 & 43.8 & 8.3 \\
\hline 370 & $\mathrm{~J} 142021.37+533900.8$ & 215.0890 & 53.6502 & 0.883 & 21.372 & 3.2 & 44.0 & 43.9 & 8.5 \\
\hline 371 & $\mathrm{~J} 141123.42+521331.7$ & 212.8476 & 52.2255 & 0.472 & 19.571 & 9.5 & 44.1 & $\ldots$ & 8.1 \\
\hline 373 & $\mathrm{~J} 141859.75+521809.7$ & 214.7490 & 52.3027 & 0.884 & 19.626 & 12.6 & 44.9 & $\ldots$ & 8.8 \\
\hline 375 & $\mathrm{~J} 141530.66+520439.5$ & 213.8777 & 52.0776 & 0.647 & 19.718 & 13.3 & 44.5 & $\ldots$ & 8.7 \\
\hline 376 & $\mathrm{~J} 140814.29+531855.8$ & 212.0596 & 53.3155 & 0.933 & 21.148 & 2.4 & 44.4 & $\ldots$ & 7.8 \\
\hline 377 & $\mathrm{~J} 142043.53+523611.4$ & 215.1814 & 52.6032 & 0.337 & 19.767 & 7.3 & 43.4 & 43.6 & 7.9 \\
\hline 378 & $\mathrm{~J} 141320.05+520527.9$ & 213.3335 & 52.0911 & 0.600 & 19.851 & 4.2 & 43.8 & 43.9 & 7.9 \\
\hline 382 & $\mathrm{~J} 140801.35+530915.9$ & 212.0056 & 53.1544 & 0.837 & 21.035 & 1.9 & 43.9 & 44.0 & 8.8 \\
\hline 385 & $\mathrm{~J} 142124.36+532312.5$ & 215.3515 & 53.3868 & 0.826 & 21.278 & 3.1 & 44.0 & 44.0 & 8.0 \\
\hline 392 & $\mathrm{~J} 142112.29+524147.3$ & 215.3012 & 52.6965 & 0.843 & 20.443 & 6.4 & 44.3 & 44.0 & 8.2 \\
\hline 393 & $\mathrm{~J} 141048.58+535605.2$ & 212.7024 & 53.9348 & 0.583 & 20.519 & 4.2 & 43.9 & 43.7 & 7.5 \\
\hline 399 & $\mathrm{~J} 141031.33+521533.8$ & 212.6305 & 52.2594 & 0.608 & 20.142 & 6.6 & 44.0 & 44.1 & 8.1 \\
\hline 407 & $\mathrm{~J} 142115.76+533128.7$ & 215.3157 & 53.5246 & 0.922 & 19.830 & 8.3 & 44.7 & 43.1 & 8.1 \\
\hline 421 & $\mathrm{~J} 140822.72+533437.2$ & 212.0947 & 53.5770 & 0.791 & 21.248 & 1.3 & 43.6 & 44.0 & 8.4 \\
\hline 422 & $\mathrm{~J} 140739.17+525850.7$ & 211.9132 & 52.9808 & 1.073 & 19.724 & 5.1 & $\ldots$ & $\ldots$ & $\ldots$ \\
\hline 427 & $\mathrm{~J} 140744.85+525211.5$ & 211.9369 & 52.8699 & 1.073 & 20.273 & 5.4 & $\ldots$ & $\ldots$ & $\ldots$ \\
\hline 428 & $\mathrm{~J} 141856.19+535845.0$ & 214.7341 & 53.9792 & 0.976 & 18.299 & 30.4 & 45.4 & $\ldots$ & 8.7 \\
\hline 437 & $\mathrm{~J} 141723.08+540641.5$ & 214.3462 & 54.1115 & 0.856 & 19.791 & 12.0 & 44.7 & $\ldots$ & 8.3 \\
\hline 438 & $\mathrm{~J} 140733.13+531254.1$ & 211.8880 & 53.2150 & 0.826 & 19.698 & 5.8 & 44.5 & 44.2 & 8.6 \\
\hline 439 & $\mathrm{~J} 141049.76+540040.6$ & 212.7073 & 54.0113 & 0.834 & 21.126 & 3.2 & 44.0 & 44.0 & 7.8 \\
\hline 440 & $\mathrm{~J} 142209.14+530559.8$ & 215.5381 & 53.0999 & 0.754 & 19.527 & 15.6 & 44.7 & 44.1 & 9.1 \\
\hline 443 & $\mathrm{~J} 141811.08+520618.0$ & 214.5462 & 52.1050 & 1.122 & 20.923 & 4.3 & $\ldots$ & $\ldots$ & $\ldots$ \\
\hline 450 & $\mathrm{~J} 142217.19+530211.2$ & 215.5716 & 53.0364 & 0.896 & 20.585 & 6.3 & 44.4 & 43.6 & 8.6 \\
\hline 453 & $\mathrm{~J} 141058.78+520712.2$ & 212.7449 & 52.1200 & 0.391 & 20.001 & 4.2 & 43.6 & 43.3 & 8.4 \\
\hline 457 & $\mathrm{~J} 141417.13+515722.6$ & 213.5714 & 51.9563 & 0.604 & 20.288 & 2.1 & 43.4 & 43.5 & 8.1 \\
\hline 460 & $\mathrm{~J} 141634.36+515849.3$ & 214.1432 & 51.9804 & 0.990 & 19.293 & 15.8 & 45.0 & $\ldots$ & 8.9 \\
\hline 465 & $\mathrm{~J} 142008.27+521646.9$ & 215.0345 & 52.2797 & 1.059 & 18.188 & 31.6 & $\ldots$ & $\ldots$ & $\ldots$ \\
\hline
\end{tabular}


Table 1

(Continued)

\begin{tabular}{|c|c|c|c|c|c|c|c|c|c|}
\hline RMID & $\begin{array}{c}\text { SDSS } \\
\text { Identifier }\end{array}$ & $\begin{array}{c}\text { R.A. }^{a} \\
(\mathrm{deg}) \\
(\mathrm{J} 2000)\end{array}$ & $\begin{array}{c}\text { Decl. }^{\mathrm{a}} \\
(\mathrm{deg}) \\
(\mathrm{J} 2000)\end{array}$ & $z^{\mathrm{a}}$ & $i \mathrm{mag}^{\mathrm{a}}$ & $\begin{array}{l}\mathrm{MED}^{\mathrm{b}} \\
\mathrm{S} / \mathrm{N}\end{array}$ & $\begin{array}{c}\text { AGN } \\
\log \lambda L_{\lambda 5100}{ }^{c} \\
\left(\mathrm{erg} \mathrm{s}^{-1}\right)\end{array}$ & $\begin{array}{c}\text { Host } \\
\log \lambda L_{\lambda 5100} \mathrm{c} \\
\left(\mathrm{erg} \mathrm{s}^{-1}\right)\end{array}$ & $\begin{array}{c}\log M_{\mathrm{BH}, \mathrm{SE}}{ }^{\mathrm{c}} \\
\left(M_{\odot}\right)\end{array}$ \\
\hline 469 & $\mathrm{~J} 142106.27+534407.0$ & 215.2761 & 53.7353 & 1.006 & 18.307 & 24.9 & 45.4 & $\ldots$ & 9.0 \\
\hline 472 & $\mathrm{~J} 141104.87+520516.8$ & 212.7703 & 52.0880 & 1.080 & 18.982 & 19.1 & $\ldots$ & $\ldots$ & $\ldots$ \\
\hline 478 & $\mathrm{~J} 140726.47+524710.5$ & 211.8603 & 52.7862 & 0.957 & 19.495 & 7.4 & 44.6 & 44.1 & 8.7 \\
\hline 480 & $\mathrm{~J} 140752.37+523622.3$ & 211.9682 & 52.6062 & 0.996 & 21.361 & 1.7 & 44.2 & 44.6 & 8.2 \\
\hline 489 & $\mathrm{~J} 142120.78+534235.8$ & 215.3366 & 53.7099 & 1.002 & 20.834 & 3.0 & 44.4 & $\ldots$ & 7.9 \\
\hline 492 & $\mathrm{~J} 141154.13+520023.4$ & 212.9755 & 52.0065 & 0.963 & 18.953 & 17.4 & 45.0 & 43.6 & 8.7 \\
\hline 497 & $\mathrm{~J} 142236.11+530923.2$ & 215.6505 & 53.1564 & 0.511 & 19.311 & 8.7 & 44.2 & 44.2 & 9.5 \\
\hline 510 & $\mathrm{~J} 140820.78+522444.3$ & 212.0866 & 52.4123 & 0.710 & 20.780 & 4.1 & 44.1 & 43.7 & 8.3 \\
\hline 515 & $\mathrm{~J} 141808.04+520023.3$ & 214.5335 & 52.0065 & 0.805 & 20.326 & 6.2 & 44.4 & 44.2 & 8.8 \\
\hline 518 & $\mathrm{~J} 142222.79+524354.0$ & 215.5949 & 52.7317 & 0.459 & 20.069 & 4.0 & 43.9 & $\ldots$ & 7.3 \\
\hline 519 & $\mathrm{~J} 141712.30+515645.5$ & 214.3012 & 51.9460 & 0.554 & 21.537 & 1.5 & 43.2 & 43.2 & 7.4 \\
\hline 525 & $\mathrm{~J} 140929.77+535930.0$ & 212.3740 & 53.9917 & 0.863 & 19.666 & 11.9 & 44.7 & $\ldots$ & 7.6 \\
\hline 539 & $\mathrm{~J} 141816.11+541120.0$ & 214.5671 & 54.1889 & 0.846 & 20.733 & 3.4 & 44.1 & $\ldots$ & 8.7 \\
\hline 541 & $\mathrm{~J} 141852.64+520142.8$ & 214.7193 & 52.0286 & 0.440 & 20.590 & 4.7 & 43.5 & 43.3 & 7.7 \\
\hline 545 & $\mathrm{~J} 140643.27+531619.6$ & 211.6803 & 53.2721 & 0.979 & 19.770 & 12.4 & 44.9 & $\ldots$ & 9.0 \\
\hline 546 & $\mathrm{~J} 141928.58+520439.4$ & 214.8691 & 52.0776 & 1.028 & 21.368 & 2.9 & 44.6 & 44.6 & 8.1 \\
\hline 548 & $\mathrm{~J} 141553.09+541816.5$ & 213.9712 & 54.3046 & 0.731 & 20.594 & 5.9 & 44.1 & 43.9 & 7.5 \\
\hline 551 & $\mathrm{~J} 141147.06+515619.8$ & 212.9461 & 51.9388 & 0.680 & 21.522 & 5.3 & 44.0 & 43.7 & 7.7 \\
\hline 572 & $\mathrm{~J} 141809.85+515531.6$ & 214.5411 & 51.9255 & 0.990 & 19.493 & 9.5 & 44.9 & $\ldots$ & 9.1 \\
\hline 588 & $\mathrm{~J} 142304.15+524630.2$ & 215.7673 & 52.7750 & 0.998 & 18.642 & 24.8 & 45.3 & 43.4 & 8.5 \\
\hline 589 & $\mathrm{~J} 142049.28+521053.3$ & 215.2053 & 52.1815 & 0.751 & 20.740 & 8.9 & 44.4 & 43.8 & 8.5 \\
\hline 593 & $\mathrm{~J} 141623.53+514912.7$ & 214.0980 & 51.8202 & 0.990 & 19.836 & 8.9 & 44.7 & $\ldots$ & 8.1 \\
\hline 601 & $\mathrm{~J} 140904.43+540344.2$ & 212.2685 & 54.0623 & 0.658 & 20.100 & 5.1 & 44.1 & 43.6 & 9.1 \\
\hline 618 & $\mathrm{~J} 141625.25+542312.4$ & 214.1052 & 54.3868 & 0.755 & 21.432 & 2.1 & 43.6 & 43.9 & 7.7 \\
\hline 622 & $\mathrm{~J} 141115.19+515209.0$ & 212.8133 & 51.8692 & 0.572 & 19.554 & 12.2 & 44.3 & 43.7 & 8.2 \\
\hline 632 & $\mathrm{~J} 141637.17+514627.1$ & 214.1549 & 51.7742 & 0.681 & 21.587 & 1.6 & 43.6 & 43.3 & 8.2 \\
\hline 634 & $\mathrm{~J} 141135.89+515004.5$ & 212.8995 & 51.8346 & 0.650 & 20.758 & 3.8 & 44.0 & 43.6 & 7.5 \\
\hline 637 & $\mathrm{~J} 142129.26+521153.3$ & 215.3719 & 52.1981 & 0.848 & 19.046 & 13.8 & 44.8 & $\ldots$ & 7.8 \\
\hline 638 & $\mathrm{~J} 141753.58+514918.4$ & 214.4732 & 51.8218 & 0.677 & 20.654 & 5.9 & 44.2 & 44.0 & 8.4 \\
\hline 641 & $\mathrm{~J} 141405.66+514425.9$ & 213.5236 & 51.7405 & 0.805 & 21.223 & 3.1 & 44.0 & 44.0 & 8.6 \\
\hline 643 & $\mathrm{~J} 142119.53+520959.7$ & 215.3314 & 52.1666 & 0.961 & 21.154 & 3.5 & 44.2 & 44.2 & 8.5 \\
\hline 644 & $\mathrm{~J} 142301.87+523316.7$ & 215.7578 & 52.5546 & 0.845 & 20.205 & 6.0 & 44.5 & $\ldots$ & 8.8 \\
\hline 645 & $\mathrm{~J} 142039.80+520359.7$ & 215.1658 & 52.0666 & 0.474 & 19.783 & 9.2 & 44.1 & 43.2 & 8.2 \\
\hline 649 & $\mathrm{~J} 140554.86+525347.5$ & 211.4786 & 52.8965 & 0.849 & 20.485 & 4.7 & 44.3 & 44.1 & 8.0 \\
\hline 653 & $\mathrm{~J} 142346.35+531807.4$ & 215.9431 & 53.3020 & 0.883 & 20.392 & 2.7 & 44.2 & 44.1 & 8.1 \\
\hline 654 & $\mathrm{~J} 142353.92+530722.7$ & 215.9747 & 53.1230 & 0.670 & 20.937 & 3.6 & 43.8 & 43.8 & 8.2 \\
\hline 659 & $\mathrm{~J} 141528.40+514308.7$ & 213.8683 & 51.7191 & 0.922 & 19.524 & 10.9 & 44.8 & $\ldots$ & 8.3 \\
\hline 663 & $\mathrm{~J} 142346.21+532212.5$ & 215.9425 & 53.3701 & 0.674 & 20.479 & 4.3 & 44.0 & 43.5 & 8.1 \\
\hline 664 & $\mathrm{~J} 141202.26+514638.5$ & 213.0094 & 51.7774 & 0.840 & 20.665 & 9.2 & 44.6 & $\ldots$ & 8.3 \\
\hline 668 & $\mathrm{~J} 140553.05+532448.1$ & 211.4711 & 53.4134 & 0.853 & 20.408 & 3.3 & 44.3 & 44.1 & 8.3 \\
\hline 669 & $\mathrm{~J} 140548.18+525041.0$ & 211.4507 & 52.8447 & 0.839 & 20.144 & 4.8 & 44.4 & $\ldots$ & 8.5 \\
\hline 675 & $\mathrm{~J} 140843.80+540751.3$ & 212.1825 & 54.1309 & 0.918 & 19.462 & 12.3 & 44.8 & $\ldots$ & 8.8 \\
\hline 681 & $\mathrm{~J} 142235.20+522059.1$ & 215.6466 & 52.3498 & 0.972 & 21.660 & 3.2 & 44.2 & 44.0 & 8.7 \\
\hline 685 & $\mathrm{~J} 142336.77+523932.8$ & 215.9032 & 52.6591 & 0.962 & 19.871 & 9.4 & 45.0 & 44.0 & 8.5 \\
\hline 694 & $\mathrm{~J} 141706.68+514340.1$ & 214.2778 & 51.7278 & 0.532 & 19.621 & 10.3 & 44.2 & 43.6 & 7.6 \\
\hline 697 & $\mathrm{~J} 141932.16+515228.6$ & 214.8840 & 51.8746 & 1.028 & 21.223 & 2.9 & 44.6 & 44.5 & 7.9 \\
\hline 701 & $\mathrm{~J} 140715.49+535610.2$ & 211.8145 & 53.9362 & 0.683 & 19.735 & 7.7 & 44.3 & 43.9 & 8.5 \\
\hline 707 & $\mathrm{~J} 142417.22+530208.9$ & 216.0718 & 53.0358 & 0.890 & 21.154 & 2.9 & 44.1 & 43.9 & 7.6 \\
\hline 714 & $\mathrm{~J} 142349.72+523903.6$ & 215.9572 & 52.6510 & 0.921 & 19.643 & 6.9 & 44.6 & 43.9 & 8.9 \\
\hline 719 & $\mathrm{~J} 141734.88+514237.8$ & 214.3953 & 51.7105 & 0.800 & 21.662 & 2.6 & 43.8 & 43.6 & 7.9 \\
\hline 720 & $\mathrm{~J} 140518.02+531530.0$ & 211.3251 & 53.2583 & 0.467 & 19.030 & 13.7 & 44.3 & 43.4 & 8.1 \\
\hline 728 & J142419.55+531859.9 & 216.0815 & 53.3167 & 1.129 & 21.550 & 3.3 & $\ldots$ & $\ldots$ & $\ldots$ \\
\hline 733 & $\mathrm{~J} 140551.99+533852.1$ & 211.4666 & 53.6478 & 0.455 & 19.904 & 6.7 & 43.9 & 43.4 & 8.2 \\
\hline 736 & $\mathrm{~J} 140508.60+530539.0$ & 211.2858 & 53.0942 & 0.582 & 18.248 & 20.9 & 44.7 & $\ldots$ & 8.6 \\
\hline 744 & $\mathrm{~J} 141615.83+543126.4$ & 214.0659 & 54.5240 & 0.723 & 21.361 & 1.7 & 43.4 & 43.9 & 7.6 \\
\hline 746 & $\mathrm{~J} 141720.29+514032.4$ & 214.3345 & 51.6757 & 0.683 & 19.703 & 13.2 & 44.5 & 44.0 & 8.1 \\
\hline 750 & $\mathrm{~J} 140522.76+524301.7$ & 211.3448 & 52.7171 & 0.950 & 20.937 & 3.5 & 44.4 & 44.0 & 8.3 \\
\hline 756 & $\mathrm{~J} 140923.42+515120.1$ & 212.3476 & 51.8556 & 0.853 & 20.292 & 4.2 & 44.1 & 44.1 & 8.2 \\
\hline 757 & $\mathrm{~J} 141902.09+514459.1$ & 214.7587 & 51.7498 & 1.125 & 21.072 & 3.1 & $\ldots$ & $\ldots$ & $\ldots$ \\
\hline 761 & $\mathrm{~J} 142412.93+523903.4$ & 216.0539 & 52.6510 & 0.771 & 20.426 & 9.6 & 44.5 & $\ldots$ & 8.5 \\
\hline 762 & $\mathrm{~J} 141919.08+542432.8$ & 214.8295 & 54.4091 & 0.782 & 20.475 & 8.2 & 44.6 & $\ldots$ & 8.9 \\
\hline 764 & $\mathrm{~J} 142222.21+520819.3$ & 215.5925 & 52.1387 & 0.985 & 20.900 & 2.0 & 43.8 & $\begin{array}{ll}\cdots \\
44.3\end{array}$ & 8.1 \\
\hline
\end{tabular}


Table 1

(Continued)

\begin{tabular}{|c|c|c|c|c|c|c|c|c|c|}
\hline RMID & $\begin{array}{c}\text { SDSS } \\
\text { Identifier }\end{array}$ & $\begin{array}{l}\text { R.A. }^{\text {a }} \\
(\mathrm{deg}) \\
(\mathrm{J} 2000)\end{array}$ & $\begin{array}{c}\text { Decl. }^{\mathrm{a}} \\
(\mathrm{deg}) \\
(\mathrm{J} 2000)\end{array}$ & $z^{\mathrm{a}}$ & $i \mathrm{mag}^{\mathrm{a}}$ & $\begin{array}{l}\mathrm{MED}^{\mathrm{b}} \\
\mathrm{S} / \mathrm{N}\end{array}$ & $\begin{array}{c}\text { AGN } \\
\log \lambda L_{\lambda 5100} \mathrm{c} \\
\left(\mathrm{erg} \mathrm{s}^{-1}\right)\end{array}$ & $\begin{array}{c}\text { Host } \\
\log \lambda L_{\lambda 5100}{ }^{c} \\
\left(\operatorname{erg~s}^{-1}\right)\end{array}$ & $\begin{array}{c}\log M_{\mathrm{BH}, \mathrm{SE}}{ }^{\mathrm{c}} \\
\left(M_{\odot}\right)\end{array}$ \\
\hline 766 & $\mathrm{~J} 141419.84+533815.3$ & 213.5827 & 53.6376 & 0.165 & 17.461 & 41.3 & 43.7 & 43.6 & 7.5 \\
\hline 767 & $\mathrm{~J} 141650.93+535157.0$ & 214.2122 & 53.8658 & 0.527 & 20.233 & 4.1 & 43.9 & $\ldots$ & 7.5 \\
\hline 768 & $\mathrm{~J} 140915.70+532721.8$ & 212.3154 & 53.4561 & 0.258 & 18.875 & 17.8 & 43.3 & 43.7 & 8.7 \\
\hline 769 & $\mathrm{~J} 141253.92+540014.4$ & 213.2247 & 54.0040 & 0.187 & 18.702 & 16.7 & 43.0 & 43.4 & 7.9 \\
\hline 772 & $\mathrm{~J} 142135.90+523138.9$ & 215.3996 & 52.5275 & 0.249 & 18.870 & 14.8 & 43.4 & 43.6 & 7.6 \\
\hline 773 & $\mathrm{~J} 141701.93+541340.5$ & 214.2581 & 54.2279 & 1.103 & 19.262 & 13.1 & $\ldots$ & $\ldots$ & .. \\
\hline 775 & $\mathrm{~J} 140759.07+534759.8$ & 211.9961 & 53.7999 & 0.172 & 17.910 & 28.6 & 43.5 & 43.4 & 7.9 \\
\hline 776 & $\mathrm{~J} 140812.09+535303.3$ & 212.0504 & 53.8842 & 0.116 & 17.976 & 25.7 & 43.1 & 43.0 & 7.8 \\
\hline 778 & $\mathrm{~J} 141418.55+542521.8$ & 213.5773 & 54.4227 & 0.786 & 19.492 & 15.0 & 44.8 & $\ldots$ & 8.6 \\
\hline 779 & $\mathrm{~J} 141923.37+542201.7$ & 214.8474 & 54.3671 & 0.152 & 19.096 & 11.9 & 43.1 & 42.6 & 7.4 \\
\hline 781 & $\mathrm{~J} 142103.53+515819.5$ & 215.2647 & 51.9721 & 0.263 & 19.305 & 14.7 & 43.6 & 43.3 & 7.8 \\
\hline 782 & $\mathrm{~J} 141318.96+543202.4$ & 213.3290 & 54.5340 & 0.362 & 18.892 & 13.9 & 43.9 & 43.6 & 8.0 \\
\hline 783 & $\mathrm{~J} 141319.83+513718.1$ & 213.3326 & 51.6217 & 0.984 & 18.797 & 20.3 & 45.1 & $\ldots$ & 8.5 \\
\hline 788 & $\mathrm{~J} 141231.73+525837.9$ & 213.1322 & 52.9772 & 0.843 & 21.232 & 1.7 & 43.8 & 44.1 & 8.4 \\
\hline 789 & $\mathrm{~J} 141644.17+532556.1$ & 214.1840 & 53.4322 & 0.425 & 20.203 & 7.6 & 43.7 & 43.3 & 8.1 \\
\hline 790 & $\mathrm{~J} 141729.27+531826.5$ & 214.3720 & 53.3074 & 0.237 & 18.672 & 19.5 & 43.3 & 43.6 & 8.4 \\
\hline 792 & $\mathrm{~J} 141800.72+532035.9$ & 214.5030 & 53.3433 & 0.526 & 20.636 & 3.1 & 43.0 & 43.8 & 7.8 \\
\hline 797 & $\mathrm{~J} 141427.89+535309.7$ & 213.6162 & 53.8860 & 0.242 & 19.997 & 8.2 & 43.1 & 43.0 & 7.0 \\
\hline 798 & $\mathrm{~J} 141202.88+522026.1$ & 213.0120 & 52.3406 & 0.423 & 19.145 & 15.8 & 44.0 & 43.7 & 7.6 \\
\hline 804 & $\mathrm{~J} 142100.04+532139.6$ & 215.2502 & 53.3610 & 0.677 & 20.347 & 6.2 & 44.0 & 43.9 & 7.5 \\
\hline 805 & $\mathrm{~J} 140827.04+532323.3$ & 212.1127 & 53.3898 & 0.620 & 20.328 & 6.3 & 44.0 & 43.4 & 7.8 \\
\hline 808 & $\mathrm{~J} 141546.21+540954.7$ & 213.9425 & 54.1652 & 0.956 & 20.111 & 6.6 & 44.6 & 44.0 & 9.0 \\
\hline 812 & $\mathrm{~J} 141945.51+521342.2$ & 214.9396 & 52.2284 & 0.702 & 20.181 & 5.7 & 44.0 & 44.1 & 8.4 \\
\hline 813 & $\mathrm{~J} 141222.07+541020.0$ & 213.0919 & 54.1722 & 0.955 & 20.759 & 4.5 & 44.3 & 43.8 & 7.5 \\
\hline 814 & $\mathrm{~J} 140741.04+524037.0$ & 211.9210 & 52.6769 & 0.958 & 21.269 & 2.7 & 44.3 & 44.0 & 8.7 \\
\hline 822 & $\mathrm{~J} 141308.10+515210.4$ & 213.2838 & 51.8695 & 0.288 & 19.182 & 13.3 & 43.6 & 43.5 & 7.4 \\
\hline 823 & J141501.64+541930.9 & 213.7568 & 54.3253 & 1.101 & 21.069 & 2.8 & $\ldots$ & $\ldots$ & $\ldots$ \\
\hline 824 & $\mathrm{~J} 141038.11+520032.9$ & 212.6588 & 52.0091 & 0.845 & 21.526 & 2.7 & 43.9 & 43.8 & 8.5 \\
\hline 838 & $\mathrm{~J} 141731.16+542350.4$ & 214.3799 & 54.3973 & 0.855 & 21.212 & 1.9 & 43.9 & 44.1 & 8.5 \\
\hline 839 & $\mathrm{~J} 141358.91+542706.0$ & 213.4954 & 54.4517 & 0.975 & 20.644 & 2.2 & 44.2 & 44.1 & 9.1 \\
\hline 840 & $\mathrm{~J} 141645.15+542540.8$ & 214.1881 & 54.4280 & 0.244 & 18.632 & 14.1 & 43.2 & 43.5 & 8.3 \\
\hline 843 & $\mathrm{~J} 141907.91+530025.5$ & 214.7830 & 53.0071 & 0.563 & 20.846 & 2.8 & 43.3 & 43.7 & 7.6 \\
\hline 845 & $\mathrm{~J} 142321.70+532242.7$ & 215.8404 & 53.3785 & 0.273 & 19.665 & 6.9 & 42.7 & 43.5 & 7.7 \\
\hline 846 & $\mathrm{~J} 142241.37+532646.7$ & 215.6724 & 53.4463 & 0.228 & 21.540 & 1.3 & 41.8 & 42.4 & 7.5 \\
\hline 847 & $\mathrm{~J} 142324.24+533511.2$ & 215.8510 & 53.5864 & 0.758 & 19.965 & 5.9 & 44.3 & 44.4 & 9.0 \\
\hline 848 & $\mathrm{~J} 142225.62+533426.3$ & 215.6067 & 53.5740 & 0.757 & 20.806 & 3.3 & 43.7 & 44.2 & 7.8 \\
\hline
\end{tabular}

Notes.

${ }^{\mathrm{a}}$ These measurements were made as a part of the SDSS Data Release 10 (Ahn et al. 2014). The $i$ magnitudes listed are point-spread function magnitudes and have not been corrected for Galactic extinction.

${ }^{\mathrm{b}} \mathrm{MED}-\mathrm{S} / \mathrm{N}$, where $\mathrm{S} / \mathrm{N}$ is the median signal-to-noise ratio per SDSS pixel across each individual spectrum, and MED-S/N is the median across all epochs (each SDSS pixel spans $69 \mathrm{~km} \mathrm{~s}^{-1}$ ).

${ }^{\mathrm{c}}$ These measurements are taken from Shen et al. (2015b). The $M_{\mathrm{BH}, \mathrm{SE}}$ estimates were made using the Vestergaard \& Peterson (2006) prescription for $L_{5100}$.

(This table is available in its entirety in machine-readable form.)

\subsection{Spectroscopic Data}

The SDSS-RM spectroscopic data utilized in this work were all acquired with the BOSS spectrograph between 2014 January and July. The BOSS spectrograph covers a wavelength range of $\sim 3650-10400 \AA$ and has a spectral resolution of $R \sim 2000$. The processed spectra are binned to $69 \mathrm{~km} \mathrm{~s}^{-1}$ pixel $^{-1}$. We obtained a total of 32 spectroscopic epochs with a median of 4.0 days between observations and a maximum separation of 16.6 days. The observations were scheduled during dark time and occasionally had interruptions due to weather or scheduling constraints, so the cadence of the observations varies somewhat throughout the season. Figure 2 shows the actual observing cadence. The typical exposure time was $2 \mathrm{hr}$. The data were processed by the SDSS-III pipeline and then further processed using a custom flux-calibration scheme described in detail by Shen et al. (2015a). We measure the median $\mathrm{S} / \mathrm{N}$ per pixel in each epoch for each source, and we take the median among all epochs as our measure of the overall $\mathrm{S} / \mathrm{N}$ for each source, which we designate as SN-MED. The distribution of SN-MED for our sample is shown in Figure 1.

To improve our relative flux calibrations and produce light curves, we employ a series of custom procedures as implemented in a code called PrepSpec, which is described in detail by Shen et al. (2016b, 2015a). A key feature of PrepSpec is the inclusion of a time-dependent flux correction calculated by assuming that there is no intrinsic variability of the narrow emission-line fluxes over the course of the RM campaign. PrepSpec minimizes the apparent variability of the narrow lines by fitting a model to the spectra that includes intrinsic variations in both the continuum and broad emission 

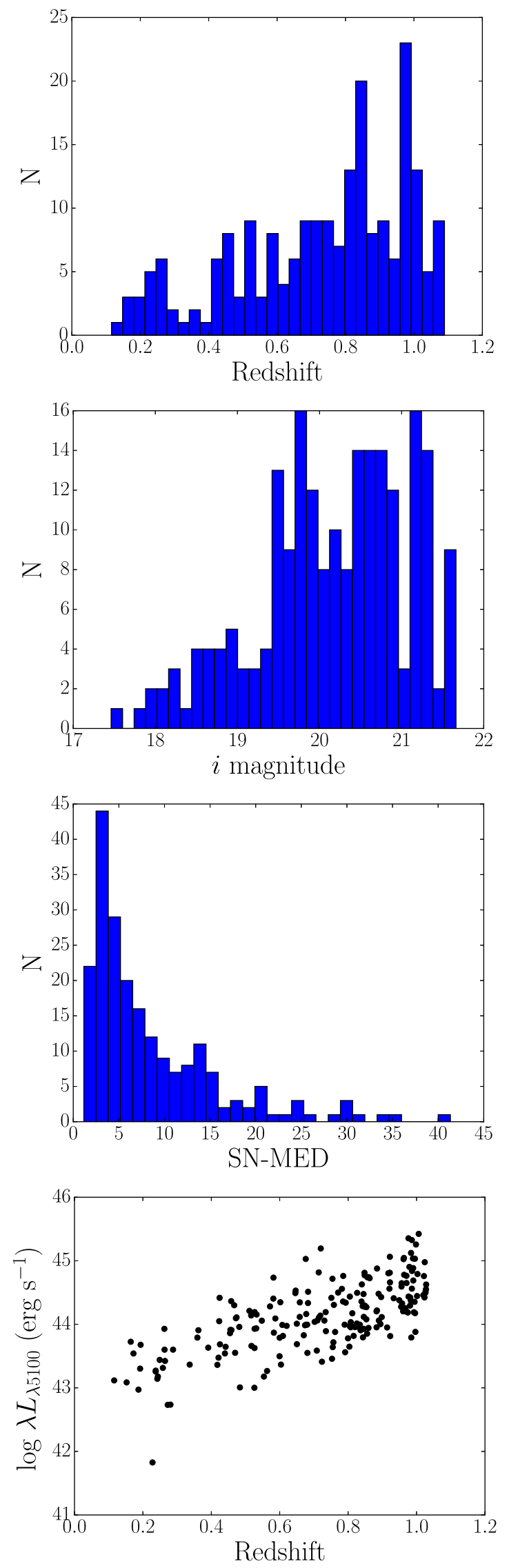

Figure 1. From top to bottom: the distributions of our sample of quasars in redshift, $i$ magnitude, median SN-MED (see Section 2.1), and $\lambda L_{\lambda 5100}$ (the hostsubtracted quasar continuum luminosity at $5100 \AA$ ) as a function of redshift.

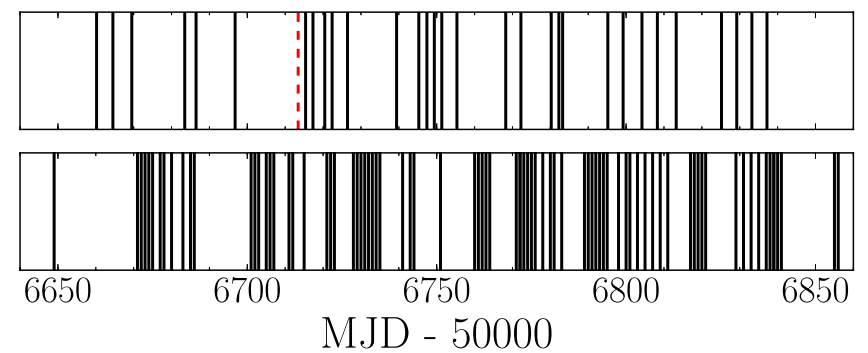

Figure 2. Observing cadence for the spectroscopic observations (top panel) and photometric observations (bottom panel). Each vertical black line represents an observed epoch. The seventh spectroscopic epoch, shown as a red dashed line, has much lower $\mathrm{S} / \mathrm{N}$ and is frequently an outlier in the light curves, so it is excluded from our analysis.

lines. PrepSpec is similar to recent spectral decomposition approaches (e.g., Barth et al. 2015), but it is optimized to fit all of the spectra of an object simultaneously and includes this flux-calibration correction. The PrepSpec model also incorporates components to account for variations in seeing and small wavelength shifts. PrepSpec produces measurements of line fluxes, mean and root mean square (rms) residual line profiles, line widths, and light curves for each of the model components. We note that the PrepSpec rms line profiles do not include the continuum and thus differ from commonly measured rms line profiles that often still include the continuum (see Section 4.2 for details).

We compute $g$ - and $i$-band synthetic photometry from each PrepSpec-scaled spectrum by convolving it with the corresponding SDSS filter response curves (Fukugita et al. 1996; Doi et al. 2010). We estimate uncertainties in the synthetic photometric fluxes as the quadratic-sum uncertainties resulting from the measurement errors in the spectrum and errors in the flux-correction factor from PrepSpec. We then later merge these light curves with the photometric light curves to improve the cadence of the continuum light curves (see Section 2.4 below). We calculate emission-line light curves directly from the PrepSpec fits.

Of the 32 available epochs, two (the third and seventh epochs) were acquired under poor observing conditions, resulting in spectra with significantly lower $\mathrm{S} / \mathrm{Ns}$ than the other epochs. Upon inspection, the seventh epoch (MJD 56713) appeared as a significant outlier in a large fraction of the light curves (more than $33 \%$ of the $\mathrm{H} \beta$ light curves). We therefore removed Epoch 7 from all of our spectroscopic light curves. There were also occasional cases of "dropped" epochs or loose fibers; these are cases where the fibers were not plugged correctly or the SDSS pipeline failed to extract a spectrum for various reasons. Loose fibers appear as significant low-flux outliers in the light curves, while dropped epochs appear as epochs with zero flux. We excluded all epochs with zero flux and epochs with loose fibers by rejecting points that were offset from the median flux by more than 5 times the normalized median absolute deviation (NMAD; e.g., Maronna et al. 2006) of the light curve (this threshold was established by visual inspection; see also Sun et al. 2015 for a discussion of dropped fibers). The final emission-line light curves of all 222 quasars are given in Table 2. We include all spectroscopic epochs in the table and mark those that were excluded from our analysis with a rejection flag $(\mathrm{FLAG}=1)$. 
Table 2

RM 005 Light Curves

\begin{tabular}{lccccc}
\hline \hline $\begin{array}{l}\text { MJD } \\
(-50000)\end{array}$ & Band $^{\mathrm{a}}$ & Telescope $^{\mathrm{b}}$ & Flux $^{\mathrm{c}}$ & Error $^{\mathrm{c}}$ & FLAG $^{\mathrm{d}}$ \\
\hline 6660.2090 & $g$ & $\mathrm{~S}$ & 16.99 & 0.33 & 0 \\
6664.5130 & $g$ & $\mathrm{~S}$ & 16.99 & 0.33 & 0 \\
6669.5003 & $g$ & $\mathrm{~S}$ & 16.90 & 0.34 & 0 \\
6686.4734 & $g$ & $\mathrm{~S}$ & 17.47 & 0.35 & 0 \\
6711.5226 & $g$ & $\mathrm{C}$ & 17.48 & 0.19 & 0 \\
6712.4684 & $g$ & $\mathrm{C}$ & 17.75 & 0.18 & 0 \\
6712.4694 & $g$ & $\mathrm{C}$ & 17.28 & 0.18 & 0 \\
6712.4703 & $g$ & $\mathrm{C}$ & 17.48 & 0.19 & 0 \\
6715.4106 & $g$ & $\mathrm{C}$ & 17.37 & 0.20 & 0 \\
6715.4116 & $g$ & $\mathrm{C}$ & 17.43 & 0.20 & 0 \\
6715.4125 & $g$ & $\mathrm{C}$ & 17.40 & 0.19 & 0 \\
6715.5388 & $g$ & $\mathrm{C}$ & 17.15 & 0.18 & 0 \\
6715.5397 & $g$ & $\mathrm{C}$ & 17.50 & 0.18 & 0 \\
6715.5407 & $g$ & $\mathrm{C}$ & 16.95 & 0.18 & 0 \\
6715.5416 & $g$ & $\mathrm{C}$ & 17.31 & 0.19 & 0 \\
6717.3345 & $g$ & $\mathrm{~S}$ & 17.15 & 0.34 & 0 \\
6720.4456 & $g$ & $\mathrm{~S}$ & 17.08 & 0.34 & 0 \\
\hline
\end{tabular}

Notes. Light curves for all 222 quasars can be found online. A portion is shown here for guidance in formatting.

${ }^{\mathrm{a}} \mathrm{H} \beta=\mathrm{H} \beta$ emission line, $\mathrm{H} \alpha=\mathrm{H} \alpha$ emission line, $g=g$ band, and $i=i$ band.

${ }^{\mathrm{b}} \mathrm{C}=$ CFHT, B $=$ Bok, $\mathrm{S}=$ SDSS.

${ }^{\mathrm{c}}$ Continuum flux densities and uncertainties are in units of $10^{-17} \mathrm{erg} \mathrm{s}^{-1} \mathrm{~cm}^{-2}$ $\AA^{-1}$. Integrated emission-line fluxes are in units of $10^{-17} \mathrm{erg} \mathrm{s}^{-1} \mathrm{~cm}^{-2}$. The fluxes are not host-subtracted.

${ }^{\mathrm{d}}$ Emission-line epochs with FLAG $=1$ were identified as outliers and excluded from the light curves in our analysis.

(This table is available in its entirety in machine-readable form.)

\subsection{Photometric Data}

In addition to spectroscopic monitoring with SDSS, we have been observing the SDSS-RM quasars in both the $g$ and $i$ bands with the Steward Observatory Bok $2.3 \mathrm{~m}$ telescope on Kitt Peak and the $3.6 \mathrm{~m}$ Canada-France-Hawaii Telescope on Maunakea. Details of the photometric observations and the subsequent data processing will be presented by K. Kinemuchi et al. (2017, in preparation). The Bok/90Prime instrument (Williams et al. 2004) used for our observations has $\mathrm{a} \sim 1^{\circ} \times 1^{\circ}$ field of view using four $4 \mathrm{k} \times 4 \mathrm{k}$ CCDs each with a plate scale of 0 "' 45 pixel $^{-1}$. Over 60 nights between 2014 January and June, largely during bright time, we obtained 31 epochs in the $g$ band and 27 epochs in $i$. The CFHT MegaCam instrument (Aune et al. 2003) also has a $\sim 1^{\circ} \times 1^{\circ}$ field of view, but has a pixel size of 0 " 187 . Over the 2014 observing period, we obtained 26 epochs in $g$ and 20 in $i$, with a few additional epochs in each band where only some of the fields were observed.

To produce photometric light curves, we adopt image subtraction as implemented in the software package ISIS (Alard \& Lupton 1998; Alard 2000). The basic procedure is to first align the images and create a reference image by combining the best images (seeing, transparency, sky background). ISIS then alters the point-spread function (PSF) of the reference image and scales the target image in overall flux calibration. It then subtracts the two to leave a "difference" image with the same flux calibration as the reference image, showing the sources that have changed in flux. We then place a PSF-weighted aperture over each source and measure the residual flux in each of the subtracted images to produce light curves. We separately produced reference images and performed the subtraction for each individual telescope, filter, CCD, and field.

After the image subtraction was complete, we removed bad measurements or outliers from the photometric light curves; these include points for sources that have fallen off the edge of the detector in certain epochs, saturated sources (either bright quasars themselves or those near a bright star, which show a large dispersion in flux in the differential photometry), and images affected by passing cirrus or other problems that deviate from the median by $>5$ times the NMAD of the light curve.

While the image-subtraction technique allows one to better compensate for changes in seeing and to separate seeingdependent aperture effects from real variability, the ISIS software takes into account only local Poisson error contributions. There are also systematic uncertainties that are not well captured by these estimates. We follow the procedure outlined by Hartman et al. (2004) and Fausnaugh et al. (2016, 2017) to apply corrections to the ISIS uncertainties. We extracted light curves for stars of magnitude similar to that of the quasars, most of which should be nonvariable. After eliminating the few variable stars, we determine an error-rescaling factor necessary for each standard star light curve to be consistent with a constant-flux model and plot this factor as a function of magnitude for each $\mathrm{CCD} /$ field combination. This provides an estimated error-rescaling factor as a function of magnitude, which we fit as a polynomial and multiply the error estimates by. Scale factors were typically about a factor of two, but range from $\sim 1$ for fainter sources to $\sim 10$ for the brightest sources. We did not apply scale factors less than 1 (i.e., we did not reduce any uncertainties from their ISIS-reported values).

\subsection{Light Curve Intercalibration}

We have several individual photometric light curves (one for each telescope/field/CCD observation) and a single synthetic photometric light curve (produced from the spectra) in each band for each quasar. For our analysis, it is necessary to place all of the $g$ - and $i$-band light curves from all CCDs/telescopes/ fields on the same flux scale; this intercalibration accounts for different detector properties, different telescope throughputs, and other properties specific to the individual telescopes involved. We assume that the time lag between the $g$ and $i$ band is much smaller than we are able to resolve with our data and thus can be treated as zero for intercalibration purposes.

We performed this intercalibration using the Continuum REprocessing AGN MCMC (CREAM) software recently developed by Starkey et al. (2016). CREAM uses Markov chain Monte Carlo (MCMC) techniques to model the light curves, assuming that the continuum emission is emitted from a central location and is reprocessed by more distant gas (see Starkey et al. 2016 for a thorough discussion of the technique). CREAM fits a model driving light curve $X(t)$ to the $g$ - and $i$-band light curves $f_{\nu}(\lambda, t)$ with an accretion-disk response function $\psi(\tau \mid \lambda)$. The model is

$$
f_{j}(\lambda, t)=\bar{F}_{j}(\lambda)+\Delta F_{j}(\lambda) \int_{0}^{\infty} \psi(\tau \mid \lambda) X(t-\tau) d \tau,
$$

where each telescope $j$ is assigned an offset $\bar{F}_{j}(\lambda)$ and flux scaling parameter $\Delta F_{j}(\lambda)$. The offset and scaling parameters control the intercalibration of the $g$ or $i$ light curves, from multiple telescopes, onto the same scale. 
These parameters are optimized in the MCMC fit, and the rescaled $g$ and $i$ light curves are calculated from the original light curves using

$$
f_{j, \text { new }}(\lambda, t)=\left(f_{j, \text { old }}(\lambda, t)-\bar{F}_{j}\right) \frac{\Delta F_{\mathrm{REF}}}{\Delta F_{j}}+\bar{F}_{\mathrm{REF}},
$$

where the subscript REF indicates the reference telescope/filter combination, and $j$ is calculated for all telescopes at each $g$ or $i$ wavelength. CREAM was initially designed to calculate interband continuum lags by fitting the accretion-disk response function $\psi(\tau \mid \lambda)$. This function is not required in the merging process here-we are only interested in the intercalibration parameters $\bar{F}_{j}(\lambda)$ and $\Delta F_{j}(\lambda)$. We therefore alter CREAM such that it has a delta function response at zero lag $\psi(\tau \mid \lambda)=\delta(\tau-0)$ for the continuum light curves in each $g$ and $i$ filter.

CREAM's MCMC algorithm also rescales the nominal error bars using an extra variance, $V_{j}$, and scale factor parameters, $f_{j}$, for each telescope (Starkey et al. 2017). The rescaled error bars are

$$
\sigma_{\mathrm{ij}}=\sqrt{\left(f_{j} \sigma_{\text {old, ij }}\right)^{2}+V_{j}},
$$

where $i=1 \ldots N_{j}$ is an index running over the $N_{j}$ data points for telescope $j$. The likelihood function $L_{j}$ penalizes high values of $V_{j}$ and $f_{j}$ in the MCMC chain and is given by

$$
-2 \ln L_{j}=N_{j} \ln (2 \pi)+\sum_{i=1}^{N_{j}}\left[\ln \sigma_{\mathrm{ij}}^{2}+\left(\frac{D_{\mathrm{ij}}-M_{\mathrm{ij}}}{\sigma_{\mathrm{ij}}}\right)^{2}\right],
$$

for data $D_{i j}$ and model $M_{i j}$. This approach provides an additional check or correction on the uncertainties for our continuum light curves.

The resulting improved "merged" light curves from CREAM are used in our RM time-series analysis. Figure 3 presents an example set of light curves for SDSS J141625.71+535438.5. The final, intercalibrated light curves for the 222 quasars are provided in Table 2 .

\section{Time-series Analysis}

\subsection{Lag Measurements}

Most prior RM measurements have been based upon crosscorrelation methods and simple linear interpolation between observations (e.g., Peterson et al. 2004). However, over the past several years, more sophisticated procedures have been developed that model the statistically likely behavior of the light curves in the gaps between observations (e.g., JAVELIN, $\mathrm{Zu}$ et al. 2011; and CREAM, Starkey et al. 2016). These procedures provide three key improvements over linear interpolation. Most importantly, their light curves have higher uncertainties in the interpolated regions compared to the observed light curve points, in contrast to the smaller uncertainties between points when using simple linear interpolation. JAVELIN and CREAM also use a damped random walk (DRW) model for the variability, matching observations (e.g., Kelly et al. 2009; Kozłowski et al. 2010; MacLeod et al. 2010). Finally, they use the same continuum DRW model fit, with a transfer function, to describe the broad-line light curves. This is essentially a prior that the BLR reverberates (although it allows either a positive or negative reverberation delay). This

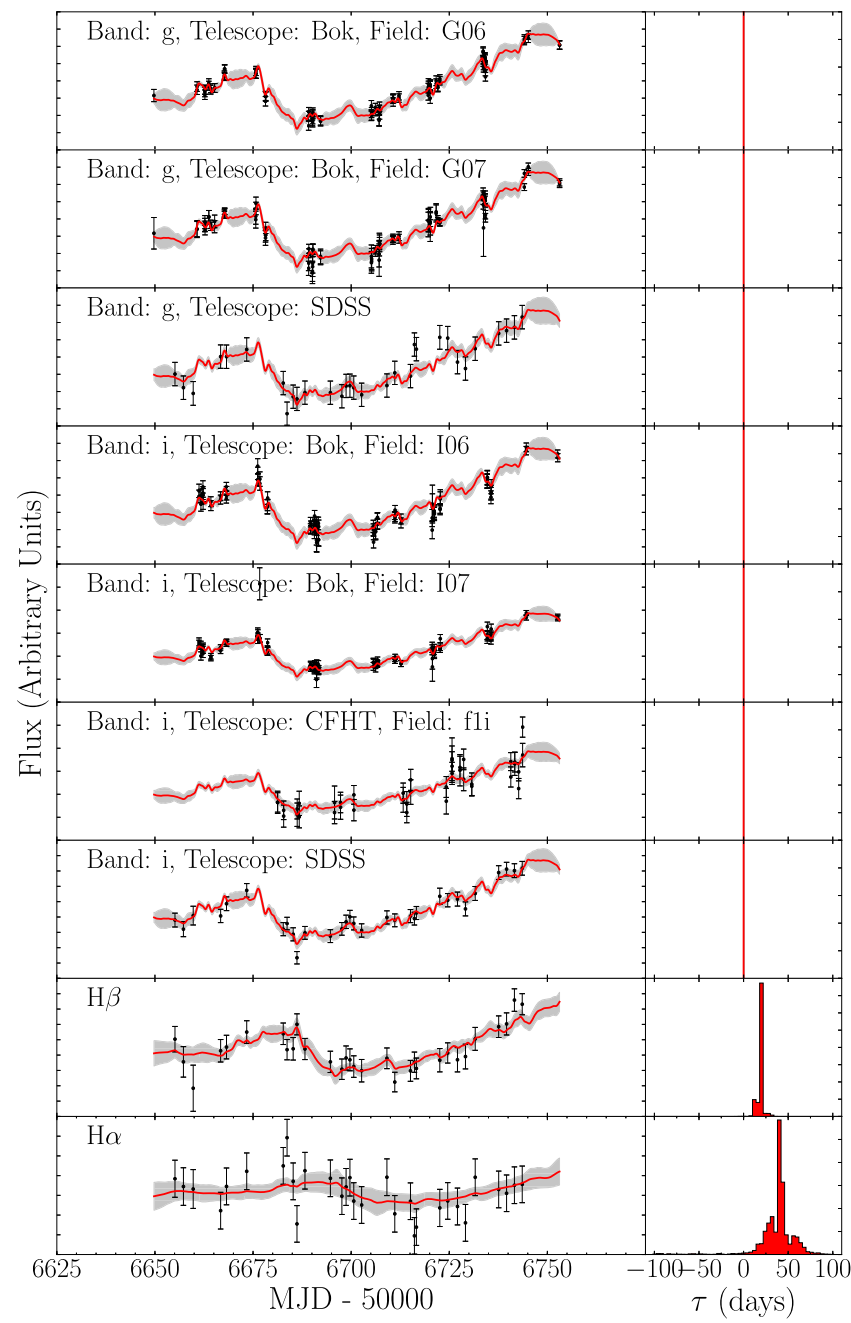

Figure 3. CREAM model fits to the light curves for SDSS J141625.71 +535438.5 (RMID 272, $z=0.263$ ) as a demonstration of the intercalibration technique. Each left panel shows an individual premerged light curve (black points) with the CREAM model fit and uncertainties in red and gray, respectively. The right panels display the corresponding CREAM-calculated posterior distribution of observed-frame time lags calculated for each light curve's response function $\psi(\tau)$. The time lag between the photometric light curves and the synthetic spectroscopic light curves is fixed to zero in order to intercalibrate the data.

assumption is the basic reason that reverberation mapping is possible, although recent observations have also identified periods of nonreverberating variability in NGC 5548 (Goad et al. 2016).

We performed our time-series analysis using all three of these methods, with the goal of comparing and contrasting the results from simple interpolation/cross-correlation and different prescriptions for statistical modeling of light curves. All of our time-series analysis is performed in the observed frame, and measured time delays are later shifted into the rest frame. Because our light curves span only about 200 days, we restrict our search to lags from -100 to +100 days. For larger and smaller lags, the overlap between the two light curves is reduced to less than half, making it harder to judge the validity of identifying correlated features. Future data spanning multiple years will soon be able to provide more reliable estimates for longer lags.

The most common methods to measure RM time lags are the interpolated cross-correlation function (ICCF; e.g., Gaskell \& 
Peterson 1987; Peterson et al. 2004) and the discrete correlation function (DCF; Edelson \& Krolik 1988) or $z$-transformed DCF (zDCF; Alexander 1997). The DCF has been shown to perform best when large numbers of points are present; for cases with lower sampling such as our data, it is better to use the ICCF (White \& Peterson 1994). The zDCF was designed to mitigate some of the issues with the DCF; however, for this study we opted to use the ICCF, as it is more traditionally used, and a detailed comparison between the ICCF and zDCF is not yet available in the literature. The ICCF method works as follows: for a given time delay $\tau$, we shift the time coordinates of the first light curve by $\tau$ and then linearly interpolate the second light curve to the new time coordinates, measuring the crosscorrelation Pearson coefficient $r$ between the two light curves using overlapping points. We next shift the second light curve by $-\tau$ and interpolate the first light curve, and average the two values of $r$. This process is repeated over the entire range of allowed $\tau$, evaluating $r$ at discrete steps in $\tau$. This procedure allows the measurement of $r$ as a function of $\tau$, called the ICCF. The centroid ( $\tau_{\text {cent }}$ ) of the ICCF is measured using points surrounding the maximum correlation coefficient $r_{\max }$ out to $r \geqslant 0.8 r_{\max }$, as is standard for ICCF analysis (e.g., Peterson et al. 2004).

We calculated ICCFs and $\tau_{\text {cent }}$ for our entire sample of quasars using an interpolation grid spacing of 2 days, calculating the ICCF between -100 and 100 days. Following Peterson et al. (2004), we estimate the uncertainty in $\tau_{\text {ICCF }}$ using Monte Carlo simulations that employ the flux randomization/random subset sampling (FR/RSS) method. Each Monte Carlo realization randomly selects a subset of the data and alters the flux of each point on the light curves by a random Gaussian deviate scaled to the measurement uncertainty of that particular point. We then calculate the ICCF for the altered set of light curves and measure $\tau_{\text {cent }}$ and $\tau_{\text {peak }}$. This procedure is repeated 5000 times to obtain the cross-correlation centroid distribution (CCCD), and the uncertainties are determined from this distribution. We adopt the median of the distribution as the best $\tau_{\text {ICCF }}$ measurement after some modifications and the removal of aliases (described below in Section 3.2). Many previous studies adopted the centroid as measured from the actual ICCF rather than the median from the CCCD. However, we use the median of the CCCD because in the case of light curves with lower time sampling, the ICCF centroid can often be an outlier in the CCCD, suggesting that the median of the CCCD is a better characterization of the true lag. However, we do note that for our data, results using the centroid of the ICCF are nearly identical to measurements using the median of the CCCD.

We used the modeling code JAVELIN (Zu et al. 2011, 2013) as our primary time-series analysis method. Rather than linearly interpolating between light curve points, JAVELIN models the light curves as an autoregressive process using a DRW model and treats the emission-line light curves as scaled, shifted, and smoothed versions of the continuum light curves. The DRW model is observed to be a good description of quasar variability within the time regime relevant to our study (e.g., Kelly et al. 2009; Kozłowski et al. 2010, 2016; MacLeod et al. 2010, 2012), so it is an effective prior to describe the light curve between observations. JAVELIN builds a model of both light curves and simultaneously fits a transfer function, maximizing the likelihood of the model and computing uncertainties using the (Bayesian) Markov chain Monte Carlo technique. The advantage of a method such as JAVELIN over the ICCF is that it replaces linear interpolation with a statistically and observationally motivated model of how to interpolate in time. The JAVELIN lag measurement takes into account the (increased) uncertainty associated with the interpolation between data points while including the statistically likely behavior of the intrinsic light curve. When multiple light curves of different emission lines are available, JAVELIN can model them simultaneously, which improves its performance and helps to eliminate multiple solutions.

The time span of our campaign observations ( $\sim 190$ days) is shorter than the typical damping timescale of a quasar ( 200-1000 days; Kelly et al. 2009; MacLeod et al. 2012; Sun et al. 2015), so JAVELIN is unable to constrain this quantity with our data (e.g., Kozłowski 2017). We thus fix the JAVELIN DRW damping timescale to be 300 days (the exact choice of timescale does not matter as long as it is longer than the baseline of our data). We use a top-hat transfer function that is parameterized by a scaling factor, width, and time delay (which we denote as $\tau_{\mathrm{JAV}}$ ) with the width fixed to 2.0 days and the time delay restricted to be within -100 to 100 days. The best-fit lag and its uncertainties are calculated from the posterior lag distribution from the MCMC chain.

As discussed in Section 2.4, Starkey et al. (2016) recently developed an alternate approach to modeling light curves and measuring time delays called CREAM. In addition to merging the $g$ and $i$ light curves, CREAM is also able to infer simultaneously the $\mathrm{H} \alpha$ and $\mathrm{H} \beta$ lags. To achieve this, we assign a delta function response to the $\mathrm{H} \alpha$ and $\mathrm{H} \beta$ lags such that $\psi(\tau \mid \lambda)=\delta\left(\tau-\tau_{\mathrm{BLR}}\right)$, where $\tau_{\mathrm{BLR}}$ is a fitted parameter in the MCMC chain along with the intercalibration parameters $\bar{F}_{j}(\lambda)$ and $\Delta F_{j}(\lambda)$ (see Equation (2)). CREAM self-consistently accounts for the joint errors in calibration and merging of the light curves when determining the lag. The CREAM posterior probability histograms for the $\tau_{\text {BLR }}$ parameters are shown for an example source in Figure 3. We again measure the best-fit lag (here denoted $\tau_{\text {CREAM }}$ ) from the posterior lag distribution for the corresponding emission line.

All RM methods operate under the assumption that the broad-line region responds to a "driving" continuum light curve; this assumption is generally well justified given that most monitored AGNs have been observed to reverberate. However, there is a question as to whether or not the $5100 \AA$ continuum emission is a good proxy for the actual emission driving the emission-line response. We discuss this possible issue in Section 4.3.

\subsection{Alias Identification and Removal}

Examinations of the CCCD or posterior lag distributions from JAVELIN or CREAM frequently reveal a clear highsignificance peak in the distribution accompanied by additional lower-significance peaks. In general, the presence of multiple peaks or a broad distribution of lags can indicate that the lag is not well constrained. In some cases, however, one peak is clearly strongest, and the additional weaker peaks are simply aliases resulting from the limited cadence and duration of the light curves. Aliases can sometimes be comparable in strength to the correct time lag, and they often appear in light curves with multiple peaks or troughs. These aliases can skew the $\tau$ measurements or produce uncertainties that are extremely large. It is therefore necessary to identify and remove aliases or 
additional secondary peaks to obtain the best lag measurement and associated uncertainty.

Multiple CCCD peaks have been a common feature of previous RM observations, but alias removal in these singleobject campaigns was typically applied by visual inspection in an ad hoc way (B. Peterson 2017, private communication). We instead developed a quantitative technique for alias rejection, appropriate for multiobject RM surveys like SDSS-RM. First, we applied a weight on the distribution of $\tau$ measurements in the posterior probability distributions that takes into account the number of overlapping spectral epochs at each time delay. If the true lag is so large that shifting by $\tau$ leaves no overlap between the two light curves, then we have a prior expectation that the true lag $\tau$ is not detectable with these data. If shifting one light curve by $\tau$ leaves $N(\tau)$ data points in the overlap region, we may expect to be able to detect $\tau$ with a prior probability that is an increasing function of $N(\tau)$. We define this weight $P(\tau)=[N(\tau) / N(0)]^{2}$, where $N(0)$ is the number of overlapping points at a time delay of zero. The weight on each $\tau$ measurement is thus 1 for $\tau=0$ and decreases each time a data point moves outside the data overlap region when the light curve is shifted, eventually reaching zero when there is no overlap. Lags with few overlapping points are less likely to be reliable, since at fixed correlation coefficient $r$ a smaller number of points leads to a higher null-probability $p$. In this way, the $N(\tau)$ prior acts as a conservative check on longer lags, requiring stronger evidence to conclude detections with less light curve overlap. We tested different exponents for $P(\tau)=(N(\tau) / N(0))^{k}$ and ultimately adopted $k=2$ based on visual inspection of the apparent lags in the light curves. Figure 4 shows an example of the effect that this weighting has on the posterior lag distributions.

To identify peaks and aliases in the posterior distribution, we smoothed the posterior lag distributions (the cross-correlation CCCD or the JAVELIN/CREAM MCMC posterior lag distributions) by a Gaussian kernel with a width of 5 days (the choice of 5 days was determined by visual inspection). The tallest peak of the smoothed distribution was then identified as the primary lag peak. We searched for local minima on either side of this primary peak and rejected all lag samples that fell outside of these local minima. The lag $\tau$ and its uncertainties were then measured as the median and normalized mean absolute deviation of the remaining lag distribution. We performed this alias-removal procedure on the JAVELIN and CREAM posteriors and the ICCF CCCDs. Figure 4 provides a demonstration of this procedure. We note that the weighting discussed above is only used to select primary peaks and their accompanying lag samples (i.e., identify the range of lags to include); we make our lag measurements from the unweighted posteriors that fall within that lag range.

\subsection{Lag-significance Criteria}

In many cases, we find no significant correlation between the two light curves or are otherwise unable to obtain a good measurement of $\tau$ (i.e., the lag is formally consistent with zero when the uncertainties are taken into account). In order to consider the lag a "significant" detection, we require the following.

1. The measured $\tau$ is formally inconsistent with zero to at least $2 \sigma$ significance (i.e., the absolute value of the lag is greater than twice its lower-bound uncertainty for
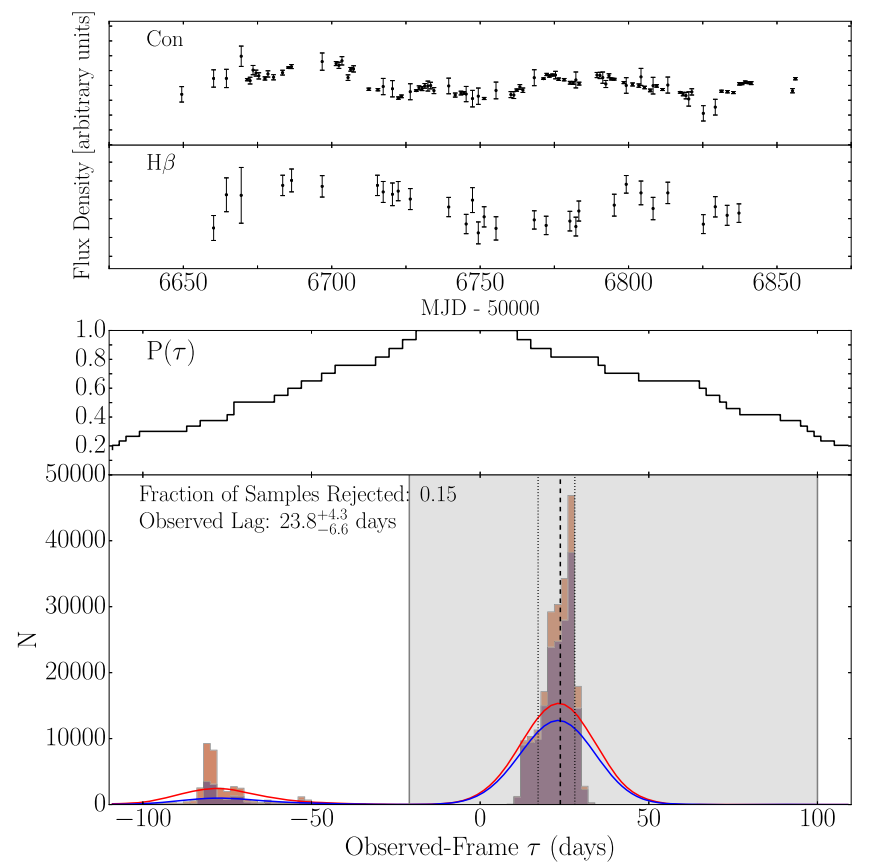

Figure 4. Light curves and the JAVELIN posterior $\mathrm{H} \beta$ lag distribution for SDSS J141018.04+532937.5 (RMID 229, $z=0.470$ ). The top two panels show the continuum and $\mathrm{H} \beta$ light curve. For display purposes, multiple observations within a single night are averaged and shown as a single point. The third panel from the top shows $P(\tau)$ used to weight the posterior lag distribution. The pink shaded histogram shows the JAVELIN posterior lag distribution before applying the weights, and the purple shaded histogram is the posterior weighted by $P(\tau)$; see Section 3.2. The solid red and blue lines are the smoothed posterior distributions for the unweighted and weighted distributions, respectively. The gray shaded region shows the lag samples surrounding the main peak of the model distribution that were included in the final lag measurement for this source. Vertical black dashed and dotted lines indicate the measured time delay and its uncertainties, respectively, estimated from the median and the mean absolute deviation of the lag distribution within the shaded region.

positive lags and twice its upper-bound uncertainty for negative lags).

2. Less than half of the samples have been rejected during the alias-identification steps described above; if this aliasremoval system excludes more than half of the samples, this is an indication that we lack a solid measurement of $\tau$.

3. The maximum ICCF correlation coefficient, $r_{\max }$, must be greater than 0.45 . This ensures that the behavior in the two light curves is well correlated. This number was determined to remove low-quality lag measurements and retain our highest-quality detections, as determined based on visual inspections of the light curves and the posterior distributions (see Section 3.5 for details).

4. The continuum and line light curve rms variability $\mathrm{S} / \mathrm{N}$ is greater than 7.5 and 0.5 , respectively (see below). This constraint excludes lag measurements that are due to spurious correlations between noisy light curves or long, monotonic trends rather than an actual reverberation signal, and it effectively requires that there is significant short-term variability in the light curves.

This final criterion requires measurements of the continuum and line light curve variance. To parameterize this, we define the "light curve $\mathrm{S} / \mathrm{N}$ " as the intrinsic variance of the light curve about a fitted linear trend, divided by its uncertainty. First, a linear trend is fit to the light curves. Following Almaini et al. 
(2000) and Sun et al. (2015), we measure the intrinsic variance from the observed $g$-band light curves using a maximumlikelihood estimator to account for the measurement uncertainties. The rms variation that we observe in the light curves, $\sigma_{\mathrm{obs}}$, is a combination of the intrinsic variance $\sigma_{\text {int }}$ and the measurement error $\sigma_{\text {err }}$, such that $\sigma_{\text {obs }}^{2}=\sigma_{\text {int }}^{2}+\sigma_{\text {err }}^{2}$. The maximum-likelihood estimator finds the intrinsic variance that maximizes the likelihood of reproducing the observed variance given the time-dependent error. Sources with short-term variability (i.e., variability other than a smooth trend) will show an excess variance about the fitted linear model, and it is only for these sources that reliable lags can be obtained.

As with our $r_{\max }$ threshold, our chosen light curve $\mathrm{S} / \mathrm{N}$ thresholds were chosen to remove spurious lag measurements while still retaining all of our highest-quality lag detections. We note, however, that the light curve $\mathrm{S} / \mathrm{N}$ as measured here is a somewhat coarse measure of the light curve quality for the purpose of lag determination, since it is a measure of the average variability over the entire light curve rather than a measure of short-term variations suitable for a lag measurement. This is why we require a line rms variability of only 0.5 , since many $0.5<\mathrm{S} / \mathrm{N}<1$ light curves still contain significant short-term variations and a reverberation signal that meets our other criteria. Despite this, the light curve $\mathrm{S} / \mathrm{N}$ remains a useful way to flag spurious correlations between noisy light curves or long, monotonic variability.

In order to estimate the false-positive detection rate of each method, we follow Shen et al. (2016b) and investigate the relative incidence of positive and negative lags. If all lag measurements were due to noise and not due to physical processes, one would expect to find equal numbers of positive and negative lags (we assume that there is no physical reason to measure a negative lag, and thus all negative lags are due to the noise or sampling properties of our light curves). Figure 5 shows the measured $\mathrm{H} \beta \tau_{\mathrm{JAV}}$ for all 222 quasars as a function of our various detection threshold parameters. We find that there is a preference for both the detected and nondetected lag measurements to be positive, suggesting that, overall, we are measuring more physical lags. We also find that light curves with high intrinsic variability are more likely to show positivelag detections, and there is a strong preference for "significant" $\mathrm{H} \beta$ lags to be positive, which suggests that, statistically, we are detecting mostly real lag signals.

Of our significant $\mathrm{H} \beta$ lag detections from JAVELIN, 32 are positive and 2 are negative; these negative lags can be considered "false positives," as they are unphysical from an RM standpoint. Statistically speaking, this suggests that we likely have a similar number of "false positive" positive $\mathrm{H} \beta$ lags as well, which is a $6.3_{-2.1}^{+7.3} \%$ false-positive rate (calculations of uncertainties follow Cameron 2011). We thus expect on the order of 30 of our $\mathrm{H} \beta$ lag measurements from JAVELIN to be real. We observe a similar fraction of false positives in our $\mathrm{H} \alpha$ lag measurements (not pictured), with 13 significant positive lags and one significant negative lag, corresponding to a false-positive rate of $7.7_{-2.6}^{+14.0} \%$. Shen et al. (2015a) simulated the expected quality of data from the SDSS-RM program (light curve cadence, $\mathrm{S} / \mathrm{N}$, and so on) and estimated a false-positive rate of between $10 \%$ and $20 \%$, which is consistent with these estimates. Our criteria for reporting detected lags are quite stringent and are meant to be conservative: the overall preference for positive lags (both significant and insignificant) suggests that it is likely that we have "detected" lags in other

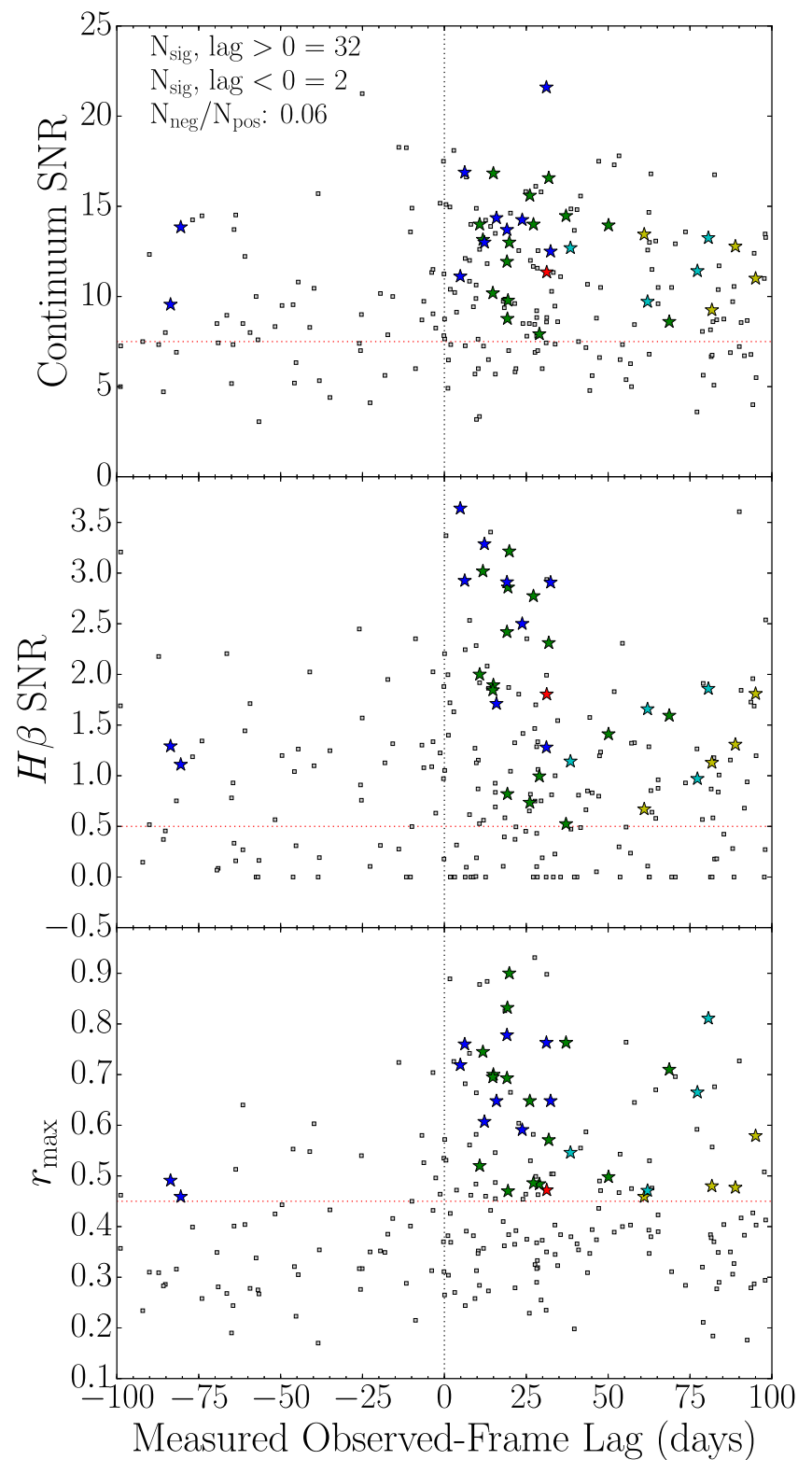

Figure 5. Measured time lags vs. parameters used to determine lag significance for our JAVELIN time-series analysis, as discussed in Section 3.3. The top panel shows the continuum light curve $\mathrm{S} / \mathrm{N}$ above a linear trend, the middle panel shows the light curve variance $\mathrm{S} / \mathrm{N}$ of the $\mathrm{H} \beta$ light curves, and the bottom panel shows the maximum correlation coefficient of the ICCF, $r_{\max }$. Lag measurements that were determined to be significant by our criteria are indicated by stars and are color coded by the quality rating assigned (see Section 3.5). Red, yellow, cyan, green, and blue represent measurements with assigned quality ratings of $1,2,3,4$, and 5 , respectively (red and yellow are the lowest-quality measurements, while blue and green are the highest). The number of significant lags greater than and less than zero is indicated in the figure text. The black vertical dotted line shows a time lag of zero, and the red horizontal dotted line shows the cutoff threshold adopted for each parameter.

objects, but the lag measurements themselves were not well constrained, so they are excluded from our analysis.

Our false-positive rate is fairly stable to reasonable changes in the parameters used to determine lag significance. Altering the threshold for continuum light curve $\mathrm{S} / \mathrm{N}$ (within the range of 6-8.5) changes the false-positive rate by less than $3 \%$ (which corresponds to just one additional false-positive measurement), and altering the line light curve $\mathrm{S} / \mathrm{N}$ within the range of $0.3-0.8$ changes the rate by less than a percent. 
The false-positive rate is more sensitive to $r_{\max }$ changes, as varying the $r_{\max }$ threshold to values within the range of $0.1-0.5$ alters the false-positive rate by $15 \%-20 \%$. Despite the stability of the false-positive rate, all three criteria place important constraints on the quality of the reported lag measurements, and thus their primary utility is in rejecting poor measurements, both positive and negative.

Having established that the majority of our significant, positive-lag detections are likely to be real, we further restrict our significant-lag sample to only those lags that are greater than zero, as a negative lag is unphysical in terms of RM. Our significant-lag detections with $\tau>0$, detected either by JAVELIN or CREAM, are reported in Table 3 . We also present the light curves and their ICCFs, CCCDs, JAVELIN model fits, JAVELIN lag posterior distributions, CREAM fits, and CREAM posterior lag distributions in Figure sets 6 and 7 for all reported positive-lag detections.

\subsection{Comparison between Different Lag-detection Methods}

One of the aims of our study was to compare results from the three different time-series analysis methods (ICCF, JAVELIN, and CREAM). The top panel of Figure 8 shows that the JAVELIN and CREAM $\mathrm{H} \beta$ lag measurements are consistent (within $1 \sigma$ ) for all but one object. Visual inspection of the outlier (RMID 622) indicates that the disagreement can be attributed to the presence of multiple peaks in the posterior distributions. There are peaks in the JAVELIN posteriors that match those from CREAM, but the peak strength ratios are reversed.

The agreement with the ICCF results is also generally quite good, as shown by the bottom panel of Figure 8 . When the lag is considered detected with the ICCF method, the $\tau_{\text {ICCF }}$ measurements are generally consistent with both JAVELIN and CREAM (i.e., all three methods agree, as these are generally our strongest cases). In the quasars with (poorly detected) ICCF lags that differ from the JAVELIN and CREAM lags by $>1 \sigma$, the posteriors of the different methods include the same peaks but at different strengths. The smaller uncertainties and larger number of well-detected lags with JAVELIN and CREAM are largely due to their use of the same (shifted, scaled, and smoothed) DRW model for both the continuum and broad-line light curves. In contrast, the ICCF assumes independent, linearly interpolated light curves for the continuum and broad lines. Well-measured light curves with high sampling result in nearly identical lag measurements from the ICCF and JAVELIN (as shown by $\mathrm{Zu}$ et al. 2011), and differences between the methods become apparent only for data sets like SDSS-RM with low cadence and noisy light curves.

Inspection of the light curves for quasars with mismatched ICCF lags (e.g., RMID 305 and 309 for $\mathrm{H} \beta$, and RMID 779 for $\mathrm{H} \alpha$ ) show that shifting the emission-line light curves by the JAVELIN and CREAM lags provides a better match to visual features repeated in both light curves than shifting by the ICCF lags does, so JAVELIN and CREAM appear to be more reliable. Jiang et al. (2017) have also run simulations with mock light curve data that suggest JAVELIN performs better than the ICCF in recovering true lags in the regime of sparsely sampled light curves. A full simulation comparing the detection completeness or efficiency for BLR lags among these different methods is currently underway (J. Li et al. 2017, in preparation). However, for our study, the above reasons and visual inspections of the light curves in Figures 6 and 7 support the use of the JAVELIN and CREAM results for our main lag detections.

Using the same positive/negative lag fraction as a falsepositive estimate, we find higher false-positive rates for CREAM and the ICCF than we did for JAVELIN. For CREAM, we measure a false-positive fraction of $16.7_{-4.2}^{+7.3} \%$ for $\mathrm{H} \beta$ (42 positive, seven negative) and $11.8_{-4.0}^{+12.2} \%$ for $\mathrm{H} \alpha$ (17 positive, two negative). For the ICCF, we measure a fraction of $25_{-7.7}^{+13} \%$ for $\mathrm{H} \beta$ (16 positive, four negative), though we do not measure any significant negative $\mathrm{H} \alpha$ lags and measure only eight positive lags, for a false-positive rate of zero (with an upper $1 \sigma$ uncertainty of $18 \%$ ).

\subsection{Lag-measurement Quality}

As suggested by our nonzero false-positive rates, it is statistically likely that a few of our lag measurements are false detections. Our objective criteria for significant-lag detection minimizes the false-positive rate and removes poor lag measurements, but does not eliminate the possibility for false detections entirely.

We tested the reliability of our lag estimates with a modified bootstrapping simulation, specifically to test whether or not our lag measurements are strongly dependent on the flux uncertainties of the light curves. For each light curve with $N$ points, we randomly draw epochs $N$ times with replacement, counting how many times each epoch is selected $\left(n_{\text {select }}\right)$. The uncertainty on the flux of each epoch is then multiplied by $1 / \sqrt{n_{\text {select }}}$ if it is selected at least once-if the epoch is not selected at all, its uncertainties are doubled. This is done 50 times for each source, creating 50 different iterations of both the continuum and $\mathrm{H} \beta$ light curves. We then run our JAVELIN analysis on the light curves with the altered uncertainties and measure the lag.

From these simulations, we compare the distribution of recovered lags with the original lag measured from the unaltered light curves and determine what percentage are consistent with the original lag to within $1 \sigma$ and $2 \sigma$. We naturally expect $68.3 \%$ of the resampled lags to be consistent to within $1 \sigma$ and $95 \%$ to be consistent to within $2 \sigma$. On average, $81 \%$ of the bootstrap simulations are within $1 \sigma$ of the original lag measurement, and $87 \%$ are within $2 \sigma$. This indicates that the JAVELIN lag estimates are robust against the uncertainties in the estimated errors in the light curve fluxes.

While we have shown that our lag measurements are generally robust, visual inspection leads us still to believe that some lags are more likely to be real than others, so we have assigned quality ratings to each of our lag measurements based on several different factors. The quality ratings range from 1 to 5 , with 1 being the poorest-quality measurements and 5 being the highest-quality detections. When assigning these quality ratings, we paid particular attention to the following.

1. The unimodality of the posterior distribution: How smooth is this distribution? Are there many other peaks beyond the main peak, or perhaps a lot of low-level noise?

2. Agreement between different methods: Do all three methods (ICCF, CREAM, and JAVELIN) result in consistent lags? In about two-thirds of our detections, our procedure yielded detected lags using JAVELIN or CREAM but not using the ICCF. Our statistical analysis (e.g., Figure 5) indicates these lags are real in the 
Table 3

SDSS-RM Observed-frame Lag Detections

\begin{tabular}{|c|c|c|c|c|c|c|c|c|c|c|}
\hline \multirow[b]{2}{*}{ RMID } & \multirow[b]{2}{*}{$z$} & \multicolumn{5}{|c|}{$\mathrm{H} \beta$ Results } & \multicolumn{4}{|c|}{$\mathrm{H} \alpha$ Results } \\
\hline & & $\begin{array}{l}\tau_{\text {ICCF }} \\
\text { (days) }\end{array}$ & $\begin{array}{c}\tau_{\mathrm{JAV}} \\
\text { (days) }\end{array}$ & $\begin{array}{c}\tau_{\text {CREAM }} \\
\text { (days) }\end{array}$ & $\begin{array}{c}\text { Quality }^{a} \\
\text { Rating }\end{array}$ & $\begin{array}{l}\tau_{\mathrm{S} 16}^{\mathrm{b}} \\
\text { (days) }\end{array}$ & $\begin{array}{l}\tau_{\text {ICCF }} \\
\text { (days) }\end{array}$ & $\begin{array}{c}\tau_{\mathrm{JAV}} \\
\text { (days) }\end{array}$ & $\begin{array}{c}\tau_{\text {CREAM }} \\
\text { (days) }\end{array}$ & $\begin{array}{l}\text { Quality }^{a} \\
\text { Rating }\end{array}$ \\
\hline 016 & 0.848 & $55.0_{-9.2}^{+9.3}$ & $64.5_{-34.6}^{+21.5}$ & $59.2_{-28.6}^{+21.4}$ & 3 & $\ldots$ & $\ldots$ & $\ldots$ & $\ldots$ & $\ldots$ \\
\hline 017 & 0.456 & $32.7_{-15.9}^{+15.4}$ & $37.1_{-8.5}^{+15.8}$ & $36.7_{-8.4}^{+4.3}$ & 4 & $\ldots$ & $65.3_{-15.4}^{+20.0}$ & $82.4_{-21.9}^{+10.6}$ & $65.7_{-13.7}^{+3.6}$ & 5 \\
\hline 021 & 1.026 & $-86.0_{-5.7}^{+10.0}$ & $88.9_{-8.8}^{+9.9}$ & $-85.8_{-3.4}^{+5.0}$ & 2 & $\ldots$ & $\ldots$ & $\ldots$ & $\ldots$ & $\ldots$ \\
\hline 033 & 0.715 & $19.0_{-15.9}^{+20.4}$ & $47.7_{-7.7}^{+11.0}$ & $45.5_{-15.1}^{+16.9}$ & 1 & $\ldots$ & $\ldots$ & $\ldots$ & $\ldots$ & $\ldots$ \\
\hline 088 & 0.516 & $\ldots$ & $\ldots$ & $\ldots$ & $\ldots$ & $\ldots$ & $84.0_{-8.3}^{+5.7}$ & $83.1_{-7.7}^{+4.4}$ & $-82.0_{-3.2}^{+3.6}$ & 3 \\
\hline 101 & 0.458 & $31.1_{-11.4}^{+5.9}$ & $31.1_{-9.3}^{+6.1}$ & $30.1_{-8.4}^{+6.8}$ & 5 & $\ldots$ & $\ldots$ & $\ldots$ & $\ldots$ & $\ldots$ \\
\hline 160 & 0.359 & $14.6_{-9.6}^{+8.9}$ & $31.3_{-4.1}^{+8.1}$ & $29.8_{-3.2}^{+5.7}$ & 3 & $\ldots$ & $16.5_{-9.2}^{+10.3}$ & $27.7_{-4.7}^{+5.3}$ & $28.5_{-3.8}^{+1.9}$ & 4 \\
\hline 177 & 0.482 & $8.3_{-6.6}^{+9.1}$ & $15.0_{-4.0}^{+18.6}$ & $33.4_{-24.3}^{+1.7}$ & 4 & $\ldots$ & $\ldots$ & $\ldots$ & $\ldots$ & $\ldots$ \\
\hline 191 & 0.442 & $14.0_{-5.8}^{+5.7}$ & $12.2_{-2.1}^{+3.6}$ & $11.0_{-1.0}^{+3.1}$ & 5 & $33.6_{-16.1}^{+3.9}$ & $26.0_{-11.8}^{+9.6}$ & $24.0_{-7.9}^{+5.9}$ & $24.2_{-8.0}^{+4.8}$ & 4 \\
\hline 215 & 0.884 & $56.0_{-16.4}^{+15.8}$ & $62.0_{-9.6}^{+10.5}$ & $59.7_{-10.4}^{+9.9}$ & 3 & $\ldots$ & $\ldots$ & $\ldots$ & $\ldots$ & $\ldots$ \\
\hline 229 & 0.470 & $21.0_{-8.7}^{+6.3}$ & $23.8_{-6.6}^{+4.3}$ & $23.0_{-6.7}^{+4.7}$ & 5 & $\ldots$ & $34.0_{-11.5}^{+19.1}$ & $32.5_{-10.7}^{+11.3}$ & $31.3_{-11.0}^{+8.3}$ & 3 \\
\hline 252 & 0.281 & $\ldots$ & $\ldots$ & $\ldots$ & $\ldots$ & $\ldots$ & $14.1_{-6.7}^{+8.1}$ & $13.0_{-2.5}^{+3.1}$ & $11.9_{-1.8}^{+2.5}$ & 5 \\
\hline 265 & 0.734 & $15.8_{-19.1}^{+10.9}$ & $14.8_{-6.8}^{+5.6}$ & $14.4_{-7.0}^{+5.7}$ & 4 & $\ldots$ & $\ldots$ & $\ldots$ & $\ldots$ & $\ldots$ \\
\hline 267 & 0.587 & $32.1_{-5.5}^{+6.9}$ & $32.4_{-3.2}^{+4.0}$ & $32.4_{-3.2}^{+2.8}$ & 5 & $29.5_{-6.0}^{+11.3}$ & $\ldots$ & $\ldots$ & $\ldots$ & $\ldots$ \\
\hline 272 & 0.263 & $21.1_{-9.0}^{+7.5}$ & $19.1_{-5.8}^{+4.0}$ & $19.5_{-3.4}^{+1.8}$ & 5 & $27.7_{-13.1}^{+10.0}$ & $42.2_{-21.1}^{+24.0}$ & $40.7_{-15.9}^{+19.7}$ & $40.2_{-17.7}^{+13.4}$ & 3 \\
\hline 300 & 0.646 & $44.0_{-16.3}^{+16.4}$ & $50.1_{-13.6}^{+6.4}$ & $54.0_{-10.4}^{+5.0}$ & 4 & $\ldots$ & $\ldots$ & $\ldots$ & $\ldots$ & $\ldots$ \\
\hline 301 & 0.548 & $21.4_{-12.8}^{+10.7}$ & $19.8_{-6.9}^{+8.9}$ & $19.0_{-3.3}^{+4.1}$ & 4 & $\ldots$ & $\ldots$ & $\ldots$ & $\ldots$ & $\ldots$ \\
\hline 305 & 0.527 & $-74.0_{-12.8}^{+22.2}$ & $81.7_{-6.2}^{+6.4}$ & $81.7_{-4.5}^{+4.1}$ & 2 & $\ldots$ & $\ldots$ & $\ldots$ & $\ldots$ & $\ldots$ \\
\hline 316 & 0.676 & $21.9_{-20.3}^{+17.3}$ & $20.2_{-3.1}^{+4.2}$ & $19.9_{-1.7}^{+2.2}$ & 3 & $\ldots$ & $\ldots$ & $\ldots$ & $\ldots$ & $\ldots$ \\
\hline 320 & 0.265 & $33.9_{-17.4}^{+10.1}$ & $31.9_{-7.2}^{+5.9}$ & $35.4_{-7.4}^{+1.8}$ & 4 & $37.4_{-19.9}^{+3.2}$ & $25.9_{-18.7}^{+18.7}$ & $25.7_{-15.6}^{+18.0}$ & $25.6_{-11.8}^{+13.3}$ & 4 \\
\hline 338 & 0.418 & $10.9_{-12.8}^{+14.2}$ & $18.5_{-9.7}^{+5.3}$ & $15.2_{-6.3}^{+7.9}$ & 4 & $\ldots$ & $\ldots$ & $\ldots$ & $\ldots$ & $\ldots$ \\
\hline 371 & 0.472 & $9.5_{-8.0}^{+12.9}$ & $10.8_{-133.1}^{+11.7}$ & $19.2_{-1.2}^{+2.1}$ & 3 & $\ldots$ & $19.4_{-20.8}^{+14.8}$ & $33.5_{-4.5}^{+1.3}$ & $33.3_{-2.2}^{+0.9}$ & 3 \\
\hline 373 & 0.884 & $34.9_{-11.1}^{+9.0}$ & $38.5_{-13.1}^{+10.5}$ & $38.7_{-11.3}^{+13.9}$ & 3 & $\ldots$ & $\ldots$ & $\ldots$ & $\ldots$ & $\ldots$ \\
\hline 377 & 0.337 & $12.0_{-15.5}^{+16.0}$ & $7.7_{-1.0}^{+0.8}$ & $7.8_{-0.7}^{+0.6}$ & 3 & $\ldots$ & $7.1_{-44.0}^{+14.3}$ & $7.9_{-1.4}^{+1.2} \& 7.7_{-0.7}^{+0.6}$ & 2 & \\
\hline 392 & 0.843 & $27.1_{-12.5}^{+6.5}$ & $26.1_{-5.5}^{+6.7}$ & $26.3_{-4.2}^{+4.1}$ & 4 & $\ldots$ & $\ldots$ & $\ldots$ & $\ldots$ & $\ldots$ \\
\hline 399 & 0.608 & $15.0_{-21.2}^{+20.7}$ & $58.0_{-1.3}^{+1.9}$ & $57.6_{-16.5}^{+1.8}$ & 2 & $\ldots$ & $\ldots$ & $\ldots$ & $\ldots$ & $\ldots$ \\
\hline 428 & 0.976 & $-80.0_{-11.2}^{+11.4}$ & $31.2_{-3.7}^{+11.9}$ & $32.3_{-7.5}^{+24.3}$ & 1 & $\ldots$ & $\ldots$ & $\ldots$ & $\ldots$ & $\ldots$ \\
\hline 457 & 0.604 & $24.0_{-21.9}^{+9.2}$ & $24.0_{-13.9}^{+6.0}$ & $25.0_{-8.2}^{+5.2}$ & 3 & $\ldots$ & $\ldots$ & $\ldots$ & $\ldots$ & $\ldots$ \\
\hline 519 & 0.554 & $0.0_{-6.2}^{+4.6}$ & $19.4_{-4.1}^{+2.9}$ & $19.5_{-2.8}^{+1.5}$ & 4 & $\ldots$ & $\ldots$ & $\ldots$ & $\ldots$ & $\ldots$ \\
\hline 551 & 0.680 & $12.9_{-11.7}^{+25.4}$ & $10.8_{-2.4}^{+2.5}$ & $10.7_{-2.6}^{+2.8}$ & 4 & $\ldots$ & $\ldots$ & $\ldots$ & $\ldots$ & $\ldots$ \\
\hline 589 & 0.751 & $69.0_{-14.4}^{+18.7}$ & $80.6_{-16.6}^{+16.7}$ & $96.2_{-18.1}^{+2.9}$ & 3 & $\ldots$ & $\ldots$ & $\ldots$ & $\ldots$ & $\ldots$ \\
\hline 601 & 0.658 & $8.8_{-18.5}^{+23.4}$ & $19.2_{-7.7}^{+14.2}$ & $19.2_{-7.4}^{+11.2}$ & 4 & $\ldots$ & $\ldots$ & $\ldots$ & $\ldots$ & $\ldots$ \\
\hline 622 & 0.572 & $76.0_{-13.2}^{+19.5}$ & $77.3_{-3.2}^{+17.5}$ & $32.9_{-1.8}^{+0.6}$ & 3 & $\ldots$ & $\ldots$ & $\ldots$ & $\ldots$ & $\ldots$ \\
\hline 634 & 0.650 & $38.1_{-19.7}^{+15.8}$ & $29.0_{-12.2}^{+14.2}$ & $29.4_{-8.9}^{+12.6}$ & 4 & $\ldots$ & $\ldots$ & $\ldots$ & $\ldots$ & $\ldots$ \\
\hline 645 & 0.474 & $7.5_{-12.5}^{+9.5}$ & $27.6_{-172.4}^{+5.2}$ & $30.6_{-4.4}^{+1.3}$ & 4 & $20.9_{-11.9}^{+9.6}$ & $26.7_{-22.9}^{+9.4}$ & $35.7_{-7.7}^{+15.1}$ & $33.9_{-5.4}^{+14.7}$ & 5 \\
\hline 694 & 0.532 & $13.1_{-15.7}^{+13.4}$ & $15.9_{-4.6}^{+9.6}$ & $15.8_{-4.0}^{+9.8}$ & 5 & $21.6_{-14.6}^{+19.8}$ & $\ldots$ & $\ldots$ & $\ldots$ & $\ldots$ \\
\hline 707 & 0.890 & $0.0_{-15.7}^{+8.5}$ & $68.7_{-10.5}^{+8.5}$ & $82.7_{-10.8}^{+4.6}$ & 4 & $\ldots$ & $\ldots$ & $\ldots$ & $\ldots$ & $\ldots$ \\
\hline 720 & 0.467 & $66.0_{-15.4}^{+11.9}$ & $61.0_{-12.2}^{+21.7}$ & $59.9_{-9.6}^{+19.6}$ & 2 & $\ldots$ & $\ldots$ & $\ldots$ & $\ldots$ & $\ldots$ \\
\hline 733 & 0.455 & $\ldots$ & $\ldots$ & $\ldots$ & $\ldots$ & $\ldots$ & $74.0_{-21.8}^{+13.9}$ & $77.0_{-8.2}^{+12.7}$ & $-84.2_{-11.4}^{+13.0}$ & 2 \\
\hline 768 & 0.258 & $\ldots$ & $\ldots$ & $\ldots$ & $\ldots$ & $\ldots$ & $42.0_{-13.0}^{+17.8}$ & $52.9_{-2.7}^{+3.4}$ & $52.7_{-1.4}^{+3.5}$ & 5 \\
\hline 772 & 0.249 & $1.3_{-5.2}^{+4.7}$ & $4.9_{-1.1}^{+1.1}$ & $5.0_{-0.6}^{+0.8}$ & 5 & $\ldots$ & $9.0_{-7.0}^{+4.3}$ & $7.4_{-1.2}^{+2.0}$ & $7.4_{-0.9}^{+1.0}$ & 5 \\
\hline 775 & 0.172 & $22.1_{-12.9}^{+10.2}$ & $19.1_{-7.7}^{+15.4}$ & $17.5_{-6.3}^{+14.1}$ & 4 & $22.5_{-15.0}^{+5.0}$ & $\ldots$ & $\ldots$ & $\ldots$ & $\ldots$ \\
\hline 776 & 0.116 & $10.0_{-4.2}^{+6.3}$ & $11.8_{-2.4}^{+1.1}$ & $12.3_{-2.9}^{+0.9}$ & 4 & $\ldots$ & $8.0_{-6.3}^{+5.4}$ & $9.2_{-2.6}^{+5.5}$ & $9.3_{-2.0}^{+3.4}$ & 4 \\
\hline 779 & 0.152 & $12.1_{-10.1}^{+9.5}$ & $13.1_{-3.4}^{+1.7}$ & $13.5_{-1.8}^{+0.8}$ & 4 & $\ldots$ & $16.9_{-13.7}^{+21.6}$ & $92.4_{-7.2}^{+5.7}$ & $92.0_{-15.4}^{+6.0}$ & 2 \\
\hline 781 & 0.263 & $93.0_{-10.5}^{+3.1}$ & $95.0_{-4.1}^{+4.0}$ & $95.4_{-3.1}^{+3.6}$ & 2 & $\ldots$ & $\ldots$ & $\ldots$ & $\ldots$ & $\ldots$ \\
\hline 782 & 0.362 & $15.0_{-6.8}^{+14.4}$ & $27.2_{-4.1}^{+1.5}$ & $27.3_{-2.1}^{+1.8}$ & 4 & $\ldots$ & $\ldots$ & $\ldots$ & $\ldots$ & $\ldots$ \\
\hline 790 & 0.237 & $11.0_{-6.5}^{+6.1}$ & $9.8_{-5.2}^{+6.8}$ & $6.8_{-2.6}^{+7.1}$ & 3 & $\ldots$ & $0.9_{-5.6}^{+11.9}$ & $55.7_{-4.8}^{+27.6}$ & $55.6_{-4.8}^{+29.3}$ & 2 \\
\hline 840 & 0.244 & $8.1_{-2.3}^{+3.4}$ & $6.2_{-1.7}^{+1.9}$ & $6.1_{-1.2}^{+1.8}$ & 5 & $13.6_{-8.2}^{+26.0}$ & $11.9_{-3.7}^{+5.6}$ & $13.2_{-3.0}^{+2.9}$ & $12.9_{-2.4}^{+2.5}$ & 5 \\
\hline
\end{tabular}

Notes.

${ }^{a}$ Lag quality rating (see Section 3.5 ).

b The lag measured by Shen et al. (2016b), for comparison purposes.

statistical sense. The ICCF is likely less powerful in detecting lags in cases where we have lower $\mathrm{S} / \mathrm{N}$ or lower-cadence light curves, so generally we prefer agreement between CREAM and JAVELIN only. However, if the ICCF results are also consistent, this likely indicates a more solid measurement, so we take this 

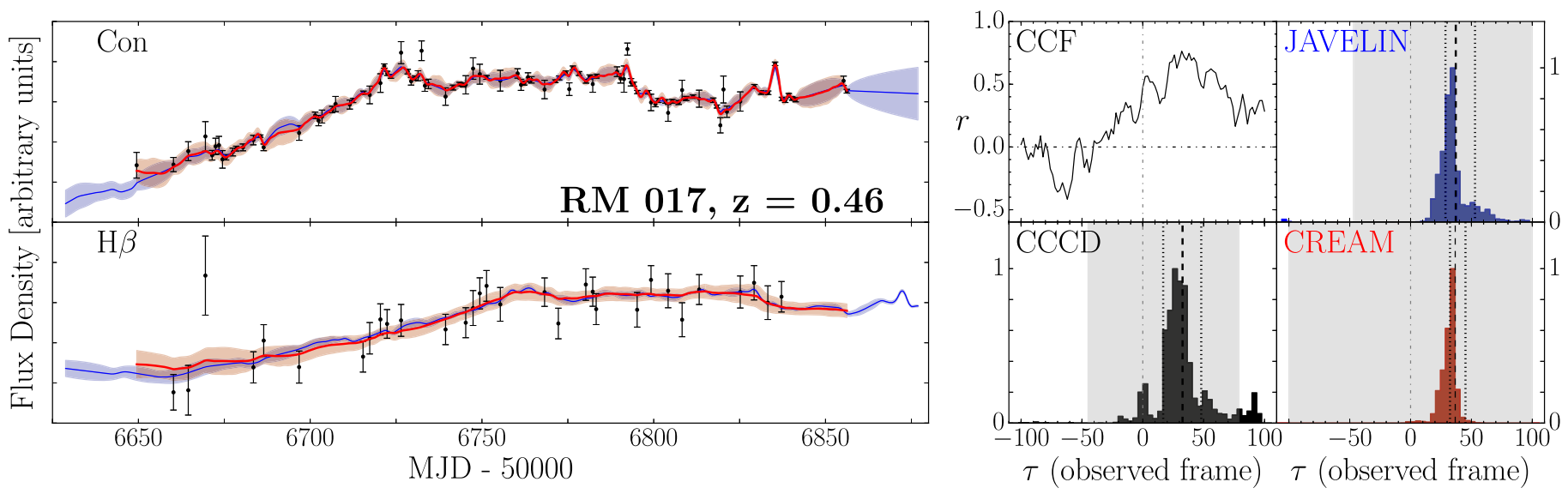

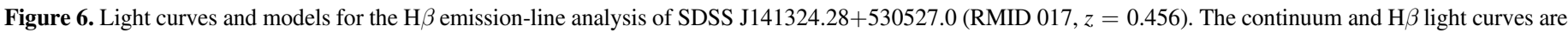

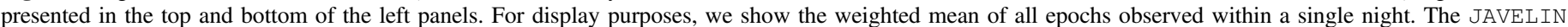

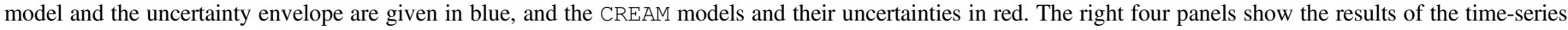

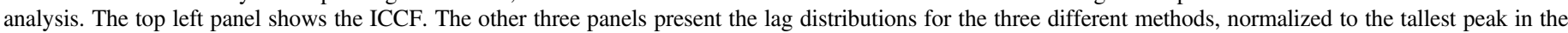

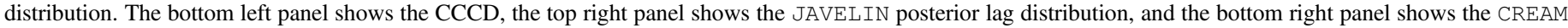

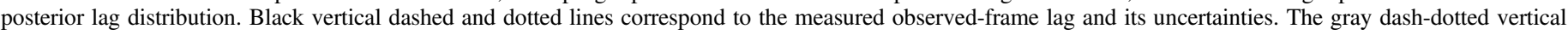

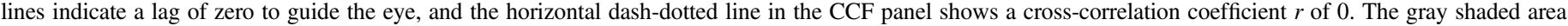

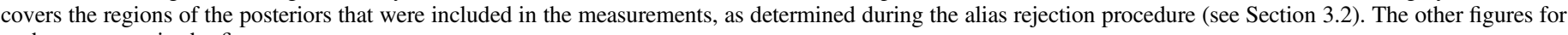
each source are in the figure set.

(The complete figure set (44 images) is available.)

into account when evaluating the quality of these measurements.

3. Light curve variability: Are there apparent short-term variability features in the continuum light curve that are also apparent in the emission-line light curve? Can we identify the lag by eye? Does the reported lag look reasonable if we shift the emission-line light curve by this lag?

4. Model fit quality: How well do the JAVELIN and CREAM model light curves match the observed light curve? Are the two model light curves in agreement with one another?

5. Bootstrapping results: What is the fraction of consistent samples from the bootstrapping described above? If enough samples are inconsistent with our original lag measurement, this indicates that the lag is less reliable, and the object is given a lower quality rating.

We include our quality assessments for each lag measurement in Table 3. We recognize that these are subjective. However, they are based on our significant past experience with RM measurements, and thus we provide them to help the reader evaluate the results.

\section{Results and Discussion}

\subsection{Lag Results}

Inspection of the light curves and posterior distributions of sources with lags that were detected by CREAM and not JAVELIN reveals that JAVELIN has a tendency to find more aliases than CREAM, particularly in light curves with a longerterm monotonic trend present in the light curve. Despite our alias-removal procedure, the presence of these aliases can cause the measurement to fail our significance criteria despite JAVELIN having measured a lag similar to CREAM. For our final $\tau$ measurements, we thus adopt $\tau_{\mathrm{JAV}}$ if the lag was detected by JAVELIN and $\tau_{\text {CREAM }}$ for the quasars in which the lag was detected by CREAM but not JAVELIN. We hereafter refer to the final adopted $\tau$ (which is equivalent to either $\tau_{\text {JAVELIN }}$ or $\left.\tau_{\text {CREAM }}\right)$ as $\tau_{\text {final }}$. This procedure yields $32 \mathrm{H} \beta$ lags from JAVELIN alone, and we add 12 more $\mathrm{H} \beta$ lags from CREAM, yielding a total of $44 \mathrm{H} \beta$ lags. Based on the $\mathrm{H} \beta$ falsepositive rates estimated for each method (see Sections 3.3 and 3.4), we expect two false positives among the JAVELIN lags and two false positives among the CREAM lags, yielding an overall number of expected false positives of four out of 44 measurements $\left(9.1_{-1.9}^{+5.6} \%\right)$. In addition, we measured $13 \mathrm{H} \alpha$ lags from JAVELIN and add five $\mathrm{H} \alpha$ lags from CREAM, yielding 18 total $\mathrm{H} \alpha$ lag measurements. Based on the $\mathrm{H} \alpha$ false-positive rate, we expect one false positive from JAVELIN and less than one from CREAM, yielding an expected 1.59 out of $18 \mathrm{H} \alpha$ lags $\left(8.8_{-2.2}^{+10.7} \%\right)$. We provide rest-frame $\tau_{\text {final }}$ measurements for all sources with detected lags in either $\mathrm{H} \beta$ or $\mathrm{H} \alpha$ in Tables 4 and 5 and show the luminosity-redshift distribution of these sources in Figure 9. We have expanded the redshift range of the RM sample out to $z \sim 1$ and increased the number of lag measurements in the sample by about two-thirds.

Shen et al. (2016b), hereafter S16, report nine $\mathrm{H} \beta$ lags from the SDSS-RM sample measured from only the spectroscopic light curves. We detect eight of them here and provide the original measurements from S16 (denoted $\tau_{\mathrm{S} 16}$ and corrected to the observed frame) in Table 3 for comparison. Our measurements for the eight detections are all consistent with theirs, but with lower uncertainties due to our addition of the photometric light curves (see Table 3 ). We find a significantly lower lag for RM 191; this is likely because of the increased cadence of our continuum light curves when the photometric monitoring was incorporated. Because of the increased cadence, we are sensitive to shorter lags and thus are able to measure the shorter lag in this object. The only source detected by S16 that we do not detect a lag for is RM 769. In our case, all three methods yielded lags that were positive but formally consistent with zero to within the uncertainties. Again, the increased cadence of the light curves is responsible for the 

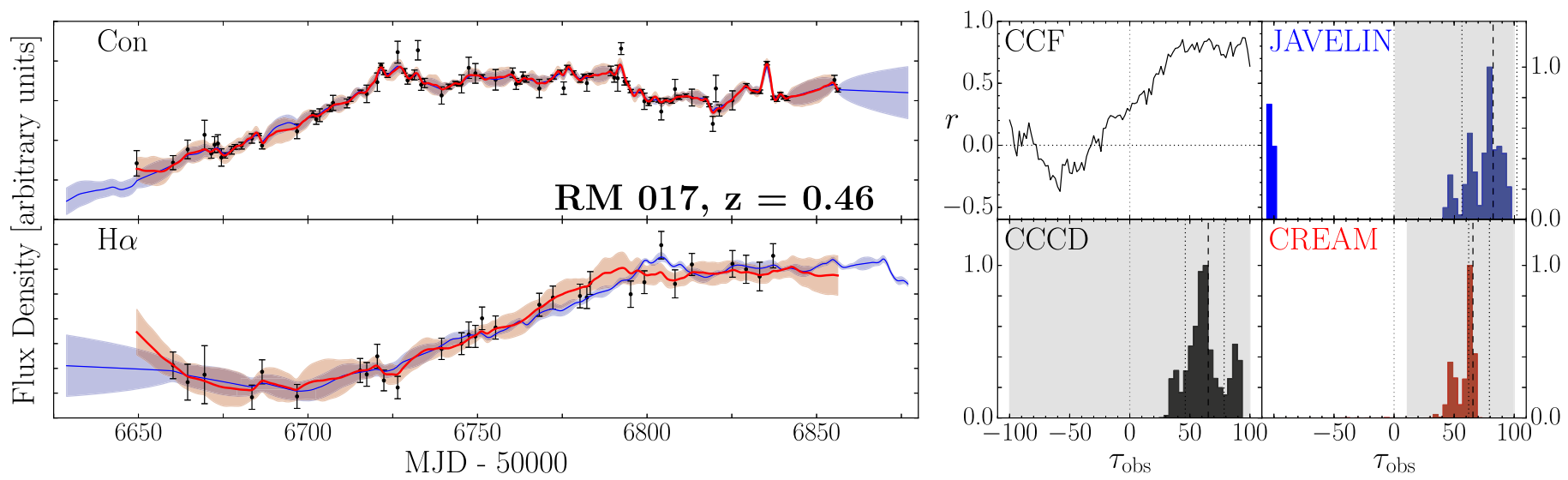

Figure 7. Light curves and output for the $\mathrm{H} \alpha$ time-series analysis for SDSS J141324.28+530527.0 (RMID 017, $z=0.456$ ). Lines and symbols are the same as in Figure 6. The other figures for each source are in the figure set.

(The complete figure set (17 images) is available.)

difference, allowing us to see that the lag is not well constrained for this source.

In 14 quasars, we measure significant lags for both $\mathrm{H} \beta$ and $\mathrm{H} \alpha$; Figure 10 compares the $\mathrm{H} \beta$ and $\mathrm{H} \alpha$ lags for those objects. We see that in all cases, the $\mathrm{H} \alpha$ lag is consistent with or larger than the $\mathrm{H} \beta$ lag; this was also reported in previous studies (e.g., Kaspi et al. 2000; Bentz et al. 2010). Larger $\mathrm{H} \alpha$ lags are expected due to photoionization predictions, with radial stratification and optical-depth effects causing the $\mathrm{H} \alpha$ emission line to appear at larger distances than $\mathrm{H} \beta$ (Netzer 1975; Rees et al. 1989; Korista \& Goad 2004); see Section 4.3 of Bentz et al. (2010) for a more detailed discussion of this phenomenon.

Shen et al. (2015b) computed the average $5100 \AA$ luminosity of most of our sources during the same monitoring period using spectral decomposition to remove host-galaxy light, allowing us to place these sources on the $R-L$ relation; we provide these luminosities in Table 1. Figure 11 presents the $R-L$ relationship measured by Bentz et al. (2013) and shows the location of our new $\mathrm{H} \beta$ lag measurements. Figure 11 also shows previous RM data from Du et al. (2016b) and the compilation of Bentz \& Katz (2015). For a consistent comparison with our SDSS-RM measurements, we use JAVELIN lags when available from the Bentz \& Katz (2015) database. Many of the lags (including the $\mathrm{Du}$ et al. 2016b data) were measured with the ICCF and so typically have larger uncertainties than JAVELIN measurements. However, the lag values themselves are consistent with ICCF measurements, and thus there are no issues when comparing measurements made with the various methods. Differences in our lag-measuring procedure (such as adopting the median of the CCCD) also yield measurements that are consistent with those using previously favored procedures, and thus these lag measurements can also be compared to lags from prior studies without issue.

Both our data and the Du et al. (2016b) super-eddington accreting massive black holes (SEAMBHs) sample have many AGNs that lie below the $R-L$ relation and its expected scatter. A similar offset from the expected $R-L$ relation was measured for the SDSS-RM quasars using composite cross-correlation methods (Li et al. 2017). At least some of the disagreement may be due to selection effects: the SDSS-RM 2014 cadence and monitoring duration limit our lag detections to less than $\sim 100$ days in the observed frame, and it is more difficult to measure the longer lags even below this limit, so we are less likely to measure lags that scatter above the $R-L$ relation. (The observations had similar cadence and duration.)

It is also possible that this offset is due to physical dependencies in the $R-L$ relation. Both the SDSS-RM and SEAMBH quasars lie at the mid-to-high-luminosity end of the $L$ distribution of the Bentz \& Katz (2015) sample of RM quasars, and it is possible that luminous quasars have different BLR radii than expected from the $R-L$ relation established from low-luminosity AGN. Du et al. (2016b) argue that the offset is caused by high accretion rates, since the most rapidly accreting SEAMBH quasars tend to be more frequently offset. We tested this hypothesis by calculating the accretion rate using the same parameterization as Du et al. (2016a, their Equation (13)). In general, our SDSS-RM quasars have much $(10-1000 \times)$ lower accretion rates than the Du et al. (2016b) sample (although our quasars have similar $L$ and $R$, they have broader line widths than the narrow-line type 1 AGNs in the SEAMBH sample). The SDSS-RM sample also does not show a clear trend between $R-L$ offset and accretion rate. It is possible that the $R-L$ offset is driven by luminosity rather than accretion rate, or by other quasar properties in which the previous RM samples were biased (e.g., Shen et al. 2015a). Fully exploring the deviations from the $R-L$ relationship will require the multiyear SDSS-RM data or careful simulations of the observational biases in order to rule out selection effects. We thus defer more detailed discussion of the $R-L$ relation to future work.

Our full sample contains 222 quasars; we have thus been able to detect lags in about $20 \%$ of them. Typical yields for traditional RM campaigns with single-object spectrographs (e.g., Fausnaugh et al. 2017) are on the order of 50\%; failure in such campaigns, which obtain very high-quality data at high cadences, is usually attributed to a lack of favorable variability behavior of the quasars. These campaigns achieve this $50 \%$ fraction through object selection (the AGNs are chosen to have strong emission lines and often are already known to show strong variability), high observing cadence (usually once per day), and high-S/N spectra. Our sample is more representative of quasars with a variety of emission-line properties and luminosities; we thus do not expect as many of our sources to vary in a favorable manner (short-term, high-amplitude variations) during the campaign. In addition, our sample is much fainter on average, which makes flux variations more difficult to detect. The cadence and length of the campaign also 

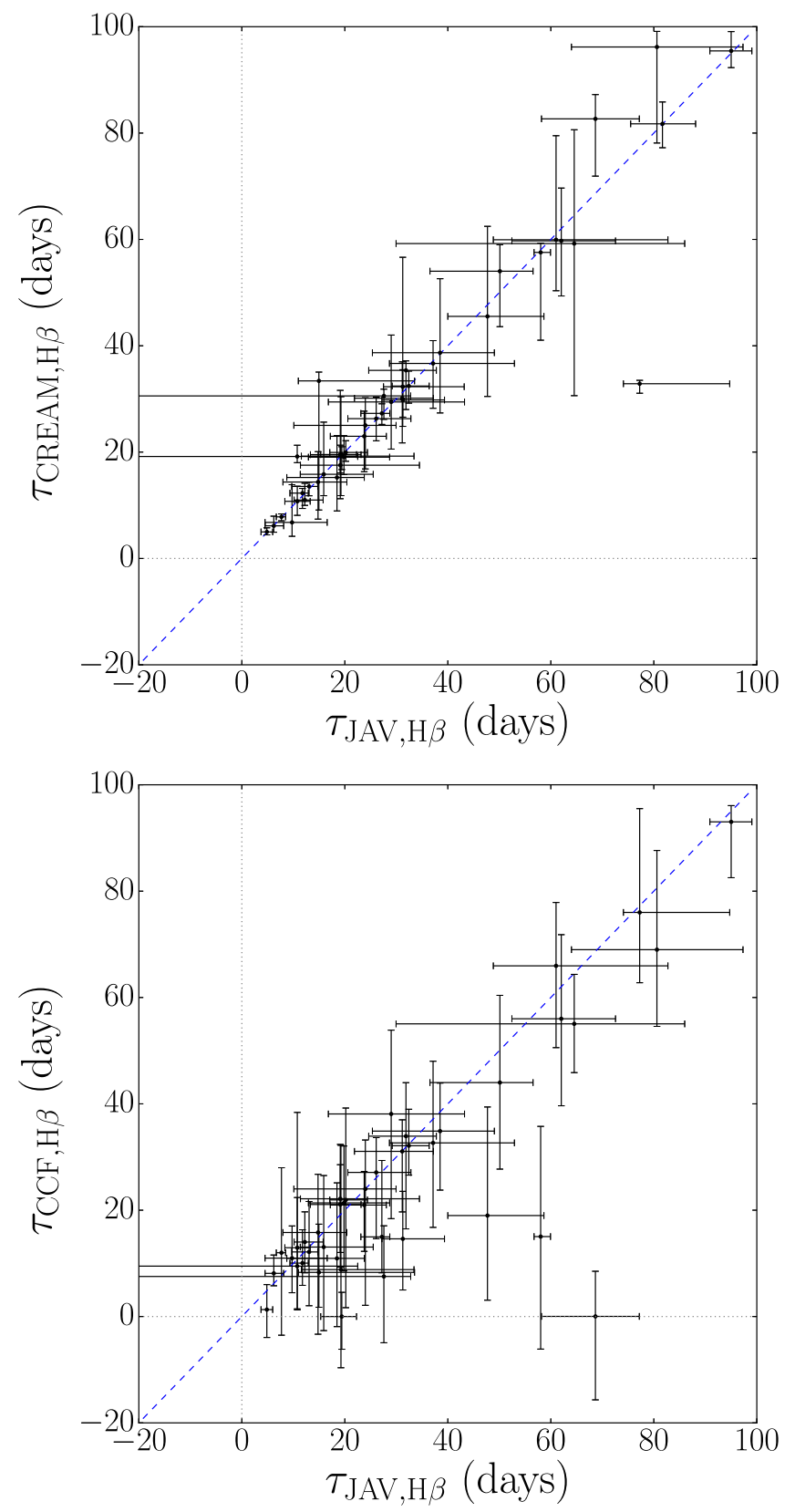

Figure 8. Comparison of the observed-frame $\mathrm{H} \beta \tau_{\text {CREAM }}$ and $\tau_{\mathrm{JAV}}$ measurements (top panel), and the $\tau_{\mathrm{JAV}}$ and $\tau_{\mathrm{ICCF}}$ measurements (bottom panel). In both panels, the blue dashed line shows a ratio of one-to-one. Gray dotted lines indicate time lags of zero along both axes to guide the eye.

affect the yield; we are unable to detect lags longer than $\sim 100$ days in the observed frame, which means that lags for the higher-luminosity quasars in our sample (expected to have $\mathrm{H} \beta$ time lags of up to $\sim 300$ days in the observed frame) are undetectable with this data set. We expect that future programs similar to SDSS-RM will similarly yield a $\sim 20 \%$ detection fraction over the first year (although the fraction may be higher for a brighter subset of quasars), with improvements if the overall cadence and monitoring length are increased.

\subsection{Black Hole Mass Measurements}

We use our $\tau_{\text {final }}$ measurements in combination with linewidth measurements from PrepSpec to compute $M_{\mathrm{BH}}$ for our sources following Equation (1). We report these line-width measurements, along with the adopted lags, calculated virial products, and $M_{\mathrm{BH}}$ measurements, for $\mathrm{H} \beta$ in Table 4 and $\mathrm{H} \alpha$ in Table 5. To calculate the virial products, we use $\sigma_{\text {line,rms }}$ measured from the rms residual spectrum, which has been shown to be a less biased estimator for $M_{\mathrm{BH}}$ than the FWHM for $\mathrm{H} \beta$-based measurements (Peterson 2011). We note that the PrepSpec rms spectrum is different from "traditional" rms spectra used in many previous studies (e.g., Kaspi et al. 2000; Peterson et al. 2004). Most prior studies include the entire spectrum, including the continuum and any blended components, in the rms spectrum computation. PrepSpec decomposes the spectra into multiple components, and the rms line profiles are measured from the broad-line model only. The resulting rms widths are different from those measured from the entire spectrum. Barth et al. (2015) examined possible sources of systematics in the rms line-width measurements and found that the inclusion of the continuum in the rms calculation can cause the line widths to be underestimated (see Barth et al. 2015, Appendix C, for details).

We propagate the uncertainties in $\tau_{\mathrm{JAV}}$ and $\sigma_{\text {line }}$ to compute the statistical uncertainties on the virial product; however, there are additional systematic uncertainties affecting $M_{\mathrm{BH}}$ measurements that have not yet been taken into account. Fausnaugh et al. (2017) calculate a 0.16 dex standard deviation in the mass of the BH in NGC 5548, which has been measured by many independent monitoring campaigns over the past 30 years (Bentz \& Katz 2015). We follow Fausnaugh et al. (2017) and add 0.16 dex uncertainties in quadrature with the statistical uncertainties in the virial product to produce our final adopted uncertainties. We adopt a scale factor $f=4.47$ (Woo et al. 2015 ) to convert the virial products to $M_{\mathrm{BH}}$. We note that the 0.16 dex systematic uncertainty is negligible compared to the systematic uncertainty in the virial scale factor $f$ (generally recognized to be on the order of $0.4 \mathrm{dex}$ ).

We also compare our $M_{\mathrm{BH}}$ measurements $\left(M_{\mathrm{BH}, \mathrm{RM}}\right)$ from the $\mathrm{H} \beta$ emission line with those measured by Shen et al. (2015b) using single-epoch spectra and the prescription of Vestergaard \& Peterson (2006), hereafter VP06, for objects with $5100 \AA$ luminosity measurements $\left(M_{\mathrm{BH}}, \mathrm{SE}\right)$. Before comparing measurements, however, we increased the reported statistical uncertainties of the single-epoch measurements by 0.43 dex to account for the intrinsic scatter measured by VP06 in the single-epoch $M_{\mathrm{BH}}$ calibration. VP06 used a higher scaling factor than our adopted value, which results in slightly higher (by $0.1 \mathrm{dex}$ ) single-epoch masses (VP06 adopt $f_{\sigma}=5.5$ and $f_{\mathrm{FWHM}}=1.4$ from Onken et al. 2004, while we adopt $f_{\sigma}=4.47$ and $f_{\text {FWHM }}=1.12$ from Woo et al. 2015).

Figure 12 shows a comparison between the two measurements. In most cases, our $M_{\mathrm{BH}}$ measurements are consistent with the single-epoch measurements given the uncertainties. The agreement is even better if a correction is applied for the different scaling factor. The scatter around a one-to-one relation among our sample is similar to the scatter seen among the Bentz \& Katz (2015) and Du et al. (2016b) samples. However, both our sample and that of Du et al. (2016b) have slightly overmassive single-epoch $M_{\mathrm{BH}}$ at low RM masses. This is consistent with the deviation seen from the $R-L$ relation (Figure 11), with smaller RM lags than expected from luminosity and the Bentz et al. (2013) relation. As before, it is possible that the differences are associated with differences in quasar properties: our sources are more luminous than those of Bentz et al. (2013), though not as rapidly accreting as the 
Table 4

Line Width, Virial Product, and $M_{\mathrm{BH}}$ Measurements for $\mathrm{H} \beta$

\begin{tabular}{|c|c|c|c|c|c|c|c|c|c|}
\hline RMID & $z$ & $\begin{array}{l}\tau_{\text {final }}{ }^{a} \\
\text { (days) }\end{array}$ & $\begin{array}{l}\sigma_{\text {line,mean }}{ }^{\mathrm{b}} \\
\left(\mathrm{km} \mathrm{s}^{-1}\right)\end{array}$ & $\begin{array}{l}\sigma_{\text {line,rms }} \mathrm{b} \\
\left(\mathrm{km} \mathrm{s}^{-1}\right)\end{array}$ & $\begin{array}{c}\mathrm{FWHM}_{\text {mean }}{ }^{\mathrm{b}} \\
\left(\mathrm{km} \mathrm{s}^{-1}\right)\end{array}$ & $\begin{array}{c}\mathrm{FWHM}_{\mathrm{rms}}^{\mathrm{b}} \\
\left(\mathrm{km} \mathrm{s}^{-1}\right)\end{array}$ & $\begin{array}{c}\mathrm{VP} \\
\left(10^{7} M_{\odot}\right)\end{array}$ & $\begin{array}{c}M_{\mathrm{BH}}{ }^{\mathrm{c}} \\
\left(10^{7} M_{\odot}\right)\end{array}$ & $\begin{array}{c}\sigma_{*}^{\mathrm{d}} \\
\left(\mathrm{km} \mathrm{s}^{-1}\right)\end{array}$ \\
\hline RM 016 & 0.848 & $32.0_{-15.5}^{+11.6}$ & $4585 \pm 50$ & $6477 \pm 54$ & $7976 \pm 42$ & $7859 \pm 112$ & $26.2_{-13.3}^{+10.4}$ & $117.3_{-59.7}^{+46.3}$ & \\
\hline RM 017 & 0.456 & $25.5_{-5.8}^{+10.9}$ & $6937 \pm 14$ & $6101 \pm 48$ & $16318 \pm 30$ & $7758 \pm 77$ & $18.5_{-5.2}^{+8.4}$ & $82.8_{-23.1}^{+37.6}$ & $191.4 \pm 3.7$ \\
\hline RM 021 & 1.026 & $43.9_{-4.3}^{+4.9}$ & $4856 \pm 536$ & $6543 \pm 34$ & $7442 \pm 5983$ & $11002 \pm 1743$ & $36.6_{-6.9}^{+7.2}$ & $163.8_{-30.8}^{+32.0}$ & $\ldots$ \\
\hline RM 033 & 0.715 & $26.5_{-8.8}^{+9.9}$ & $776 \pm 13$ & $857 \pm 32$ & $1070 \pm 30$ & $1626 \pm 243$ & $0.4_{-0.1}^{+0.2}$ & $1.7_{-0.6}^{+0.7}$ & $182.4 \pm 21.7$ \\
\hline RM 101 & 0.458 & $21.4_{-6.4}^{+4.2}$ & $1186 \pm 2$ & $976 \pm 32$ & $2220 \pm 9$ & $2135 \pm 94$ & $0.4_{-0.1}^{+0.1}$ & $1.8_{-0.6}^{+0.4}$ & $\ldots$ \\
\hline RM 160 & 0.359 & $21.9_{-2.4}^{+4.2}$ & $1773 \pm 6$ & $1909 \pm 12$ & $4399 \pm 31$ & $4183 \pm 51$ & $1.6_{-0.3}^{+0.4}$ & $7.0_{-1.3}^{+1.7}$ & $\ldots$ \\
\hline RM 177 & 0.482 & $10.1_{-2.7}^{+12.5}$ & $2541 \pm 9$ & $2036 \pm 39$ & $5277 \pm 39$ & $4930 \pm 163$ & $0.8_{-0.3}^{+1.0}$ & $3.7_{-1.1}^{+4.6}$ & $171.5 \pm 10.7$ \\
\hline RM 191 & 0.442 & $8.5_{-1.4}^{+2.5}$ & $845 \pm 12$ & $1030 \pm 18$ & $1316 \pm 94$ & $1967 \pm 76$ & $0.2_{-0.0}^{+0.1}$ & $0.8_{-0.2}^{+0.3}$ & $152.0 \pm 8.5$ \\
\hline RM 215 & 0.884 & $32.9_{-5.1}^{+5.6}$ & $7078 \pm 47$ & $7681 \pm 64$ & $13980 \pm 1935$ & $21468 \pm 2120$ & $37.9_{-8.4}^{+8.8}$ & $169.4_{-37.7}^{+39.5}$ & $\ldots$ \\
\hline RM 229 & 0.470 & $16.2_{-4.5}^{+2.9}$ & $1722 \pm 18$ & $1781 \pm 38$ & $3055 \pm 180$ & $2377 \pm 288$ & $1.0_{-0.3}^{+0.2}$ & $4.5_{-1.4}^{+1.1}$ & $130.2 \pm 8.7$ \\
\hline RM 265 & 0.734 & $8.5_{-3.9}^{+3.2}$ & $5881 \pm 103$ & $7165 \pm 36$ & $4509 \pm 71$ & $11017 \pm 109$ & $8.5_{-4.2}^{+3.5}$ & $38.2_{-18.6}^{+15.7}$ & $\ldots$ \\
\hline RM 267 & 0.587 & $20.4_{-2.0}^{+2.5}$ & $1305 \pm 6$ & $1202 \pm 33$ & $2647 \pm 23$ & $1998 \pm 75$ & $0.6_{-0.1}^{+0.1}$ & $2.6_{-0.5}^{+0.5}$ & $97.1 \pm 9.0$ \\
\hline RM 272 & 0.263 & $15.1_{-4.6}^{+3.2}$ & $1465 \pm 2$ & $1697 \pm 10$ & $2465 \pm 30$ & $4064 \pm 102$ & $0.9_{-0.3}^{+0.2}$ & $3.8_{-1.3}^{+1.0}$ & $\ldots$ \\
\hline RM 300 & 0.646 & $30.4_{-8.3}^{+3.9}$ & $1153 \pm 8$ & $1232 \pm 30$ & $2110 \pm 36$ & $2553 \pm 136$ & $0.9_{-0.3}^{+0.2}$ & $4.0_{-1.3}^{+0.8}$ & $109.4 \pm 11.9$ \\
\hline RM 301 & 0.548 & $12.8_{-4.5}^{+5.7}$ & $7061 \pm 25$ & $6259 \pm 23$ & $18920 \pm 91$ & $10477 \pm 114$ & $9.8_{-3.8}^{+4.7}$ & $43.8_{-16.8}^{+20.8}$ & $176.9 \pm 10.1$ \\
\hline RM 305 & 0.527 & $53.5_{-4.0}^{+4.2}$ & $2331 \pm 7$ & $2126 \pm 35$ & $2616 \pm 21$ & $3172 \pm 85$ & $4.7_{-0.8}^{+0.8}$ & $21.1_{-3.7}^{+3.8}$ & $150.5 \pm 7.7$ \\
\hline RM 316 & 0.676 & $11.9_{-1.0}^{+1.3}$ & $4686 \pm 12$ & $7195 \pm 40$ & $3742 \pm 6$ & $13483 \pm 141$ & $12.0_{-2.2}^{+2.3}$ & $53.7_{-9.7}^{+10.5}$ & $\ldots$ \\
\hline RM 320 & 0.265 & $25.2_{-5.7}^{+4.7}$ & $1569 \pm 9$ & $1462 \pm 26$ & $3917 \pm 28$ & $2718 \pm 80$ & $1.1_{-0.3}^{+0.3}$ & $4.7_{-1.3}^{+1.1}$ & $66.4 \pm 4.6$ \\
\hline RM 338 & 0.418 & $10.7_{-4.4}^{+5.6}$ & $2670 \pm 28$ & $2291 \pm 33$ & $4701 \pm 610$ & $5136 \pm 226$ & $1.1_{-0.5}^{+0.6}$ & $4.9_{-2.2}^{+2.7}$ & $83.3 \pm 8.3$ \\
\hline RM 371 & 0.472 & $13.0_{-0.8}^{+1.4}$ & $1484 \pm 6$ & $1443 \pm 11$ & $3458 \pm 55$ & $4123 \pm 40$ & $0.5_{-0.1}^{+0.1}$ & $2.4_{-0.4}^{+0.5}$ & $\ldots$ \\
\hline RM 373 & 0.884 & $20.4_{-7.0}^{+5.6}$ & $1726 \pm 19$ & $2491 \pm 26$ & $5582 \pm 128$ & $7211 \pm 727$ & $2.5_{-0.9}^{+0.8}$ & $11.1_{-4.2}^{+3.5}$ & $\ldots$ \\
\hline RM 377 & 0.337 & $5.9_{-0.6}^{+0.4}$ & $1648 \pm 16$ & $1789 \pm 23$ & $3555 \pm 42$ & $5654 \pm 239$ & $0.4_{-0.1}^{+0.1}$ & $1.6_{-0.3}^{+0.3}$ & $115.3 \pm 4.6$ \\
\hline RM 392 & 0.843 & $14.2_{-3.0}^{+3.7}$ & $3120 \pm 46$ & $3658 \pm 56$ & $3540 \pm 199$ & $10839 \pm 153$ & $3.7_{-1.0}^{+1.1}$ & $16.5_{-4.4}^{+5.0}$ & $77.2 \pm 25.6$ \\
\hline RM 399 & 0.608 & $35.8_{-10.3}^{+1.1}$ & $1429 \pm 23$ & $1619 \pm 38$ & $2675 \pm 60$ & $2578 \pm 112$ & $1.8_{-0.6}^{+0.3}$ & $8.2_{-2.7}^{+1.3}$ & $187.2 \pm 7.8$ \\
\hline RM 428 & 0.976 & $15.8_{-1.9}^{+6.0}$ & $6913 \pm 12$ & $7568 \pm 70$ & $11219 \pm 23$ & $7156 \pm 61$ & $17.7_{-3.5}^{+7.3}$ & $79.0_{-15.8}^{+32.7}$ & $\ldots$ \\
\hline RM 457 & 0.604 & $15.6_{-5.1}^{+3.2}$ & $2988 \pm 83$ & $2788 \pm 48$ & $6404 \pm 424$ & $7451 \pm 221$ & $2.4_{-0.9}^{+0.6}$ & $10.6_{-3.9}^{+2.8}$ & $110.0 \pm 18.4$ \\
\hline RM 519 & 0.554 & $12.5_{-2.6}^{+1.8}$ & $7008 \pm 200$ & $9475 \pm 33$ & $3740 \pm 141$ & $17614 \pm 153$ & $21.9_{-5.8}^{+4.8}$ & $97.8_{-25.9}^{+21.3}$ & $\ldots$ \\
\hline RM 551 & 0.680 & $6.4_{-1.4}^{+1.5}$ & $1194 \pm 11$ & $1298 \pm 36$ & $1887 \pm 59$ & $1638 \pm 113$ & $0.2_{-0.1}^{+0.1}$ & $0.9_{-0.3}^{+0.3}$ & $\ldots$ \\
\hline RM 589 & 0.751 & $46.0_{-9.5}^{+9.5}$ & $5424 \pm 57$ & $5013 \pm 49$ & $4553 \pm 79$ & $7625 \pm 136$ & $22.6_{-5.9}^{+5.9}$ & $100.9_{-26.3}^{+26.4}$ & $\ldots$ \\
\hline RM 601 & 0.658 & $11.6_{-4.6}^{+8.6}$ & $6705 \pm 58$ & $5284 \pm 54$ & $16168 \pm 354$ & $12673 \pm 455$ & $6.3_{-2.7}^{+4.8}$ & $28.3_{-12.2}^{+21.3}$ & $214.9 \pm 19.2$ \\
\hline RM 622 & 0.572 & $49.1_{-2.0}^{+11.1}$ & $1369 \pm 6$ & $1423 \pm 32$ & $2565 \pm 36$ & $3234 \pm 164$ & $1.9_{-0.3}^{+0.5}$ & $8.7_{-1.4}^{+2.4}$ & $122.9 \pm 9.2$ \\
\hline RM 634 & 0.650 & $17.6_{-7.4}^{+8.6}$ & $1059 \pm 25$ & $1527 \pm 22$ & $1154 \pm 42$ & $3422 \pm 491$ & $0.8_{-0.4}^{+0.4}$ & $3.6_{-1.6}^{+1.8}$ & $119.4 \pm 20.9$ \\
\hline RM 645 & 0.474 & $20.7_{-3.0}^{+0.9}$ & $1544 \pm 7$ & $1438 \pm 17$ & $3588 \pm 56$ & $3810 \pm 67$ & $0.8_{-0.2}^{+0.1}$ & $3.7_{-0.8}^{+0.6}$ & $\ldots$ \\
\hline RM 694 & 0.532 & $10.4_{-3.0}^{+6.3}$ & $845 \pm 4$ & $740 \pm 23$ & $1501 \pm 17$ & $1693 \pm 98$ & $0.1_{-0.0}^{+0.1}$ & $0.5_{-0.2}^{+0.3}$ & $\ldots$ \\
\hline RM 707 & 0.890 & $36.3_{-5.5}^{+4.5}$ & $989 \pm 20$ & $1252 \pm 11$ & $1552 \pm 95$ & $2752 \pm 90$ & $1.1_{-0.2}^{+0.2}$ & $5.0_{-1.1}^{+1.0}$ & $\ldots$ \\
\hline RM 720 & 0.467 & $41.6_{-8.3}^{+14.8}$ & $1346 \pm 4$ & $1232 \pm 16$ & $3130 \pm 23$ & $3131 \pm 44$ & $1.2_{-0.3}^{+0.5}$ & $5.5_{-1.4}^{+2.1}$ & $\ldots$ \\
\hline RM 772 & 0.249 & $3.9_{-0.9}^{+0.9}$ & $1065 \pm 14$ & $1026 \pm 14$ & $2439 \pm 33$ & $2078 \pm 35$ & $0.1_{-0.0}^{+0.0}$ & $0.4_{-0.1}^{+0.1}$ & $136.5 \pm 3.1$ \\
\hline RM 775 & 0.172 & $16.3_{-6.6}^{+13.1}$ & $1578 \pm 5$ & $1818 \pm 8$ & $3072 \pm 24$ & $5010 \pm 61$ & $1.1_{-0.5}^{+0.9}$ & $4.7_{-2.0}^{+3.9}$ & $130.4 \pm 2.6$ \\
\hline RM 776 & 0.116 & $10.5_{-2.2}^{+1.0}$ & $1501 \pm 5$ & $1409 \pm 11$ & $3700 \pm 16$ & $3111 \pm 36$ & $0.4_{-0.1}^{+0.1}$ & $1.8_{-0.5}^{+0.3}$ & $112.4 \pm 1.9$ \\
\hline RM 779 & 0.152 & $11.8_{-1.5}^{+0.7}$ & $1249 \pm 4$ & $1205 \pm 9$ & $2670 \pm 17$ & $2709 \pm 55$ & $0.3_{-0.1}^{+0.1}$ & $1.5_{-0.3}^{+0.3}$ & $57.1 \pm 4.9$ \\
\hline RM 781 & 0.263 & $75.2_{-3.3}^{+3.2}$ & $1169 \pm 5$ & $1089 \pm 22$ & $2515 \pm 26$ & $3340 \pm 82$ & $1.7_{-0.3}^{+0.3}$ & $7.8_{-1.3}^{+1.3}$ & $104.7 \pm 4.3$ \\
\hline RM 782 & 0.362 & $20.0_{-3.0}^{+1.1}$ & $1378 \pm 6$ & $1353 \pm 23$ & $3070 \pm 49$ & $2730 \pm 137$ & $0.7_{-0.2}^{+0.1}$ & $3.2_{-0.7}^{+0.5}$ & $129.5 \pm 6.7$ \\
\hline RM 790 & 0.237 & $5.5_{-2.1}^{+5.7}$ & $6813 \pm 13$ & $6318 \pm 38$ & $17112 \pm 81$ & $9448 \pm 367$ & $4.3_{-1.8}^{+4.5}$ & $19.1_{-8.0}^{+20.2}$ & $204.4 \pm 3.1$ \\
\hline RM 840 & 0.244 & $5.0_{-1.4}^{+1.5}$ & $6596 \pm 22$ & $4457 \pm 60$ & $15735 \pm 93$ & $6580 \pm 48$ & $1.9_{-0.6}^{+0.7}$ & $8.6_{-2.7}^{+3.0}$ & $164.3 \pm 3.6$ \\
\hline
\end{tabular}

Notes.

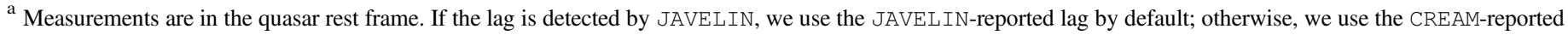
lag.

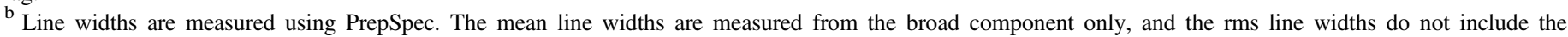
continuum.

${ }^{\mathrm{c}}$ Virial products were converted to $M_{\mathrm{BH}}$ using $f=4.47$, as measured by Woo et al. (2015).

${ }^{\mathrm{d}}$ From Shen et al. (2015b).

Du et al. (2016b) quasars. However, it is also possible that the apparent deviation is caused by selection effects associated with our limited cadence and duration, so we withhold definitive conclusions until detailed simulations of the observational biases are examined in future work.
We also compare our $M_{\mathrm{BH}}$ measurements from $\mathrm{H} \beta$ with those from $\mathrm{H} \alpha$ in Figure 13, and we find that the measurements are consistent to within the uncertainties for nearly all sources.

Figure 14 places our $M_{\mathrm{BH}}$ measurements on the $M_{\mathrm{BH}}-\sigma_{*}$ relationship. These $\sigma_{*}$ measurements were taken from Shen 
Table 5

Line Width, Virial Product, and $M_{\mathrm{BH}}$ Measurements for $\mathrm{H} \alpha$

\begin{tabular}{|c|c|c|c|c|c|c|c|c|c|}
\hline RMID & $z$ & $\begin{array}{c}\tau_{\text {final }}{ }^{\mathrm{a}} \\
\text { (days) }\end{array}$ & $\begin{array}{l}\sigma_{\text {line,mean }} \\
\left(\mathrm{km} \mathrm{s}^{-1}\right)\end{array}$ & $\begin{array}{c}\sigma_{\text {line,rms }} \\
\left(\mathrm{km} \mathrm{s}^{-1}\right)\end{array}$ & $\begin{array}{c}\mathrm{FWHM}_{\text {mean }} \\
\left(\mathrm{km} \mathrm{s}^{-1}\right)\end{array}$ & $\begin{array}{c}\mathrm{FWHM}_{\mathrm{rms}} \\
\left(\mathrm{km} \mathrm{s}^{-1}\right)\end{array}$ & $\begin{array}{c}\mathrm{VP} \\
\left(10^{7} M_{\odot}\right)\end{array}$ & $\begin{array}{c}M_{\mathrm{BH}}{ }^{\mathrm{b}} \\
\left(10^{7} M_{\odot}\right)\end{array}$ & $\begin{array}{c}\sigma_{*}^{\mathrm{c}} \\
\left(\mathrm{km} \mathrm{s}^{-1}\right)\end{array}$ \\
\hline RM 017 & 0.456 & $56.6_{-15.1}^{+7.3}$ & $4509 \pm 53$ & $4569 \pm 51$ & $4159 \pm 13$ & $5604 \pm 31$ & $23.1_{-7.2}^{+4.7}$ & $103.1_{-32.0}^{+21.2}$ & $191.4 \pm 3.7$ \\
\hline RM 088 & 0.516 & $54.8_{-5.1}^{+2.9}$ & $2449 \pm 27$ & $3320 \pm 26$ & $4451 \pm 32$ & $10290 \pm 142$ & $11.8_{-2.2}^{+2.0}$ & $52.7_{-9.8}^{+8.9}$ & $128.5 \pm 12.3$ \\
\hline RM 160 & 0.359 & $21.0_{-2.8}^{+1.4}$ & $1707 \pm 3$ & $1318 \pm 11$ & $3805 \pm 10$ & $3642 \pm 26$ & $0.7_{-0.1}^{+0.1}$ & $3.2_{-0.7}^{+0.6}$ & $\ldots$ \\
\hline RM 191 & 0.442 & $16.7_{-5.5}^{+4.1}$ & $858 \pm 6$ & $796 \pm 23$ & $2050 \pm 18$ & $1575 \pm 60$ & $0.2_{-0.1}^{+0.1}$ & $0.9_{-0.3}^{+0.3}$ & $152.0 \pm 8.5$ \\
\hline RM 229 & 0.470 & $22.1_{-7.3}^{+7.7}$ & $1528 \pm 10$ & $1738 \pm 31$ & $2271 \pm 34$ & $2103 \pm 365$ & $1.3_{-0.5}^{+0.5}$ & $5.8_{-2.1}^{+2.2}$ & $130.2 \pm 8.7$ \\
\hline RM 320 & 0.265 & $20.2_{-9.3}^{+10.5}$ & $1538 \pm 3$ & $1320 \pm 17$ & $3232 \pm 12$ & $2808 \pm 41$ & $0.7_{-0.3}^{+0.4}$ & $3.1_{-1.5}^{+1.7}$ & $66.4 \pm 4.6$ \\
\hline RM 371 & 0.472 & $22.6_{-1.5}^{+0.6}$ & $1381 \pm 4$ & $1346 \pm 13$ & $2678 \pm 12$ & $3483 \pm 44$ & $0.8_{-0.1}^{+0.1}$ & $3.6_{-0.6}^{+0.6}$ & $\ldots$ \\
\hline RM 377 & 0.337 & $5.7_{-0.5}^{+0.5}$ & $1407 \pm 10$ & $1372 \pm 40$ & $2802 \pm 17$ & $2971 \pm 114$ & $0.2_{-0.0}^{+0.0}$ & $0.9_{-0.2}^{+0.2}$ & $115.3 \pm 4.6$ \\
\hline RM 645 & 0.474 & $24.2_{-5.3}^{+10.2}$ & $1378 \pm 6$ & $1352 \pm 24$ & $2825 \pm 12$ & $3118 \pm 80$ & $0.9_{-0.2}^{+0.4}$ & $3.9_{-1.0}^{+1.7}$ & $\ldots$ \\
\hline RM 733 & 0.455 & $53.0_{-5.7}^{+8.7}$ & $1488 \pm 7$ & $1590 \pm 24$ & $3284 \pm 21$ & $3489 \pm 84$ & $2.6_{-0.5}^{+0.6}$ & $11.7_{-2.2}^{+2.7}$ & $196.9 \pm 16.6$ \\
\hline RM 768 & 0.258 & $42.1_{-2.1}^{+2.7}$ & $3428 \pm 16$ & $3232 \pm 40$ & $6213 \pm 9$ & $6279 \pm 20$ & $8.6_{-1.4}^{+1.5}$ & $38.3_{-6.4}^{+6.6}$ & $171.9 \pm 2.8$ \\
\hline RM 790 & 0.237 & $45.0_{-3.9}^{+23.7}$ & $3532 \pm 17$ & $5157 \pm 40$ & $5769 \pm 18$ & $8898 \pm 66$ & $23.3_{-4.2}^{+12.9}$ & $104.4_{-19.0}^{+57.5}$ & $204.4 \pm 3.1$ \\
\hline RM 840 & 0.244 & $10.6_{-2.4}^{+2.3}$ & $3002 \pm 45$ & $3927 \pm 30$ & $4593 \pm 14$ & $6027 \pm 19$ & $3.2_{-0.9}^{+0.9}$ & $14.3_{-4.0}^{+3.8}$ & $164.3 \pm 3.6$ \\
\hline
\end{tabular}

Notes.

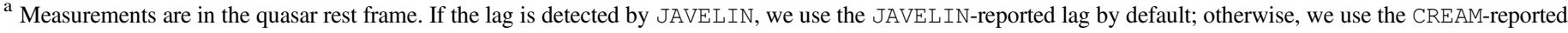
lag.

${ }^{\mathrm{b}}$ Virial products were converted to $M_{\mathrm{BH}}$ using $f=4.47$, as measured by Woo et al. (2015).

${ }^{\mathrm{c}}$ From Shen et al. (2015b).

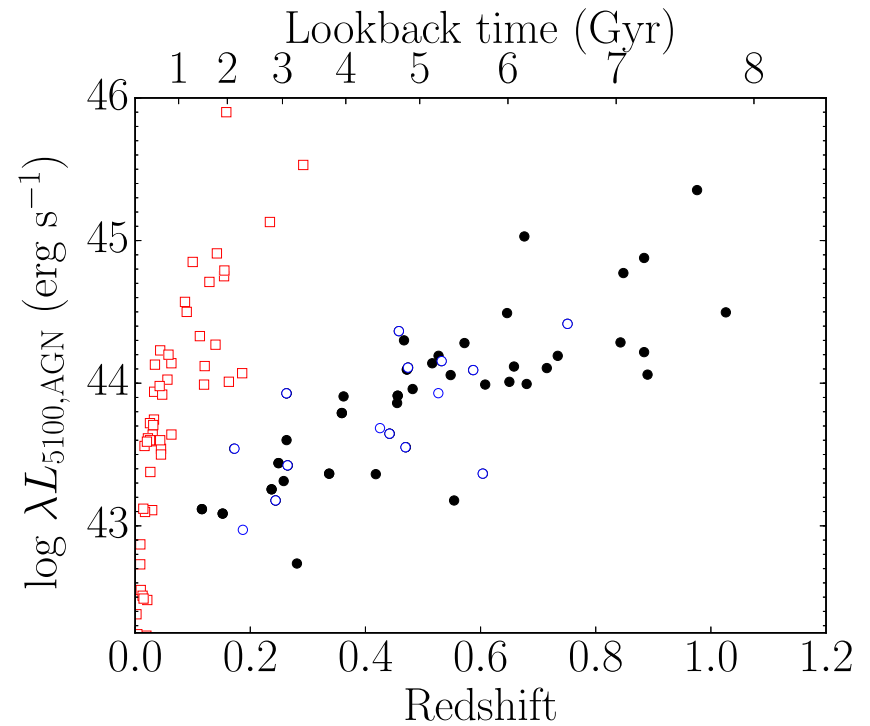

Figure 9. Distribution of redshift and $\log \lambda L_{5100}$ of the sources with detected $\mathrm{H} \beta$ lags. Red open squares represent the 42 local RM AGNs compiled by Bentz \& Katz (2015), with additions from Du et al. (2014, 2015) and Fausnaugh et al. (2017), showing average luminosities when multiple measurements exist for a single source. Blue open circles show the SDSSRM first-lags sample from Shen et al. (2016b), and black solid circles show our new measurements. Note that the Shen et al. (2016b) measurements include $\mathrm{Mg}$ II detections and that there is overlap between eight of the Shen et al. (2016b) $\mathrm{H} \beta$ measurements and our new measurements.

et al. (2015b), but they are also consistent with those measured independently by Matsuoka et al. (2015) based on a different spectral decomposition approach. Most of our measurements are consistent with the local quiescent $M_{\mathrm{BH}}-\sigma_{*}$ relation, though large uncertainties and the presence of outliers at low $\sigma_{*}$

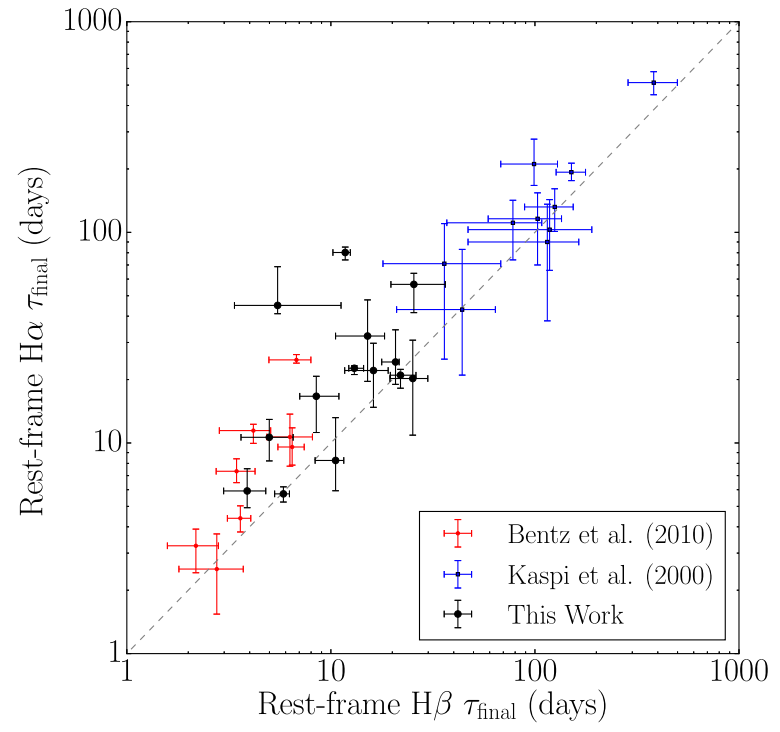

Figure 10. $\mathrm{H} \alpha$ vs. $\mathrm{H} \beta$ lag measurements for those objects where we detected significant lags for both emission lines (black solid circles). Red points represent measurements from Bentz et al. (2010), and blue squares represent measurements from Kaspi et al. (2000). The gray dashed line shows a ratio of one-to-one to guide the eye.

introduce a large amount of scatter and dilute any correlation within our sample. The four outliers at low $\sigma_{*}$ are RMIDs 320, 338, 392, and 779. All four of these lag measurements appear solid: we see visible short-term variability in the light curves, and the lags are well determined, with clean posteriors. In addition, three out of the four lags are consistent with expectations from the $R-L$ relation, further suggesting that they are robust (the fourth source, RM 392, is expected to have 


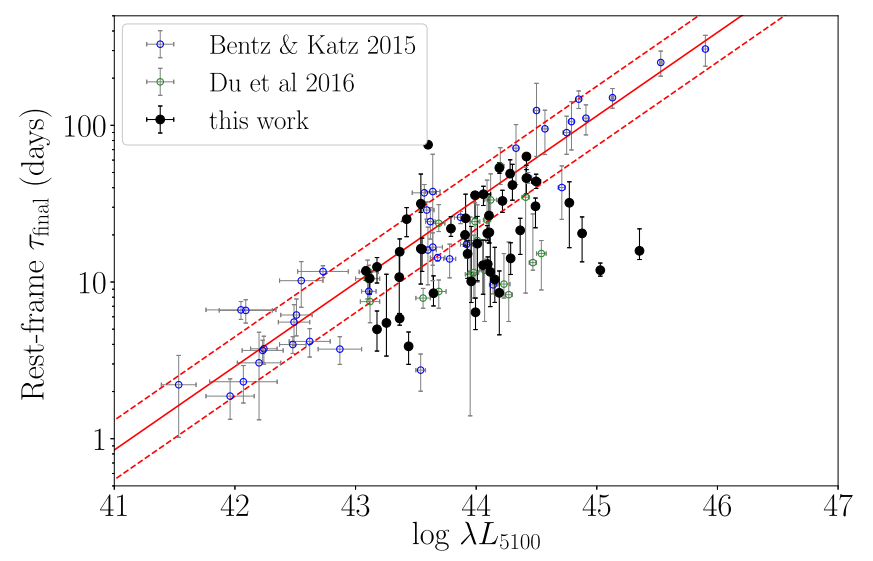

Figure 11. $\mathrm{H} \beta R-L$ relationship, with previous measurements in blue (Bentz \& Katz 2015) and green (Du et al. 2016b) and our new measurements in black. The red solid and dashed lines show the best-fit relation and its measured scatter from Bentz et al. (2013). Many of the SDSS-RM and Du et al. (2016b) lags lie below the main $R-L$ relation: this may be (at least partly) due to selection effects from our limited monitoring cadence and duration, since our survey (and that of Du et al. 2016b) is not sensitive to long lags at high luminosities. The deviation may also be a physical effect associated with a different BLR size at high luminosities, or other quasar parameters that differ between the initial Bentz et al. (2013) data set and the SDSS-RM data and Du et al. (2016b) samples.

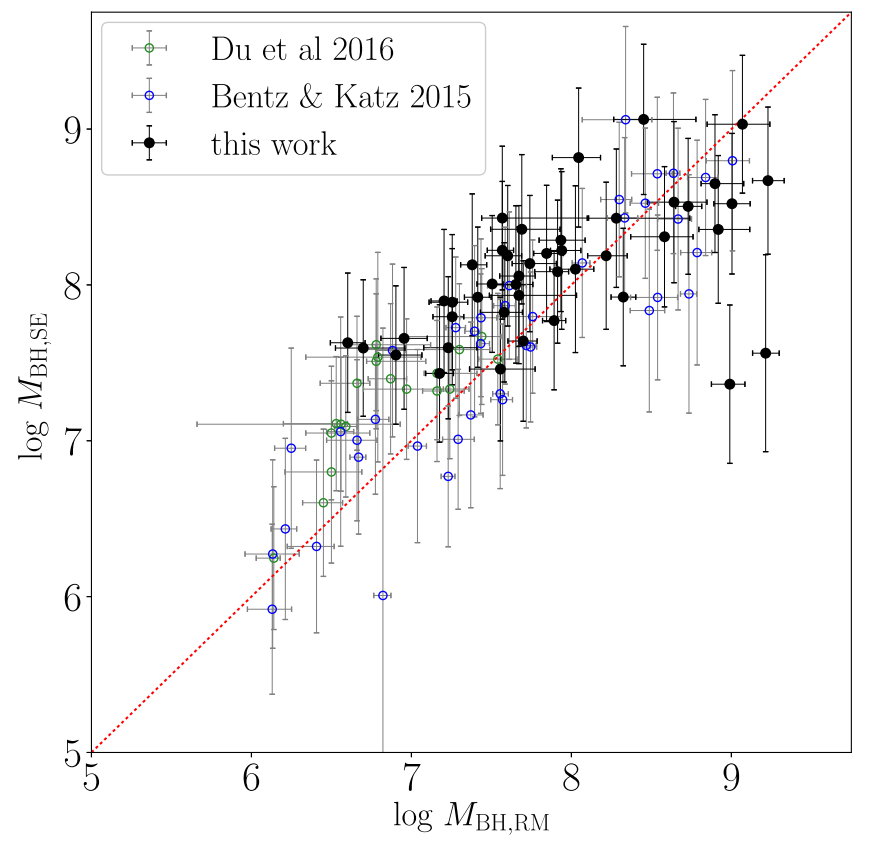

Figure 12. Comparison of $M_{\mathrm{BH}, \mathrm{SE}}$ measurements from Shen et al. (2015b) and our new measurements $\left(M_{\mathrm{BH}, \mathrm{RM}}\right)$. As in Figure 11, we show previous measurements in blue (Bentz \& Katz 2015) and green (Du et al. 2016b). The dotted red line indicates a ratio of one-to-one. Most of our quasars have consistent masses between the two methods, with some deviation for both SDSS-RM and the Du et al. (2016b) sample at low RM masses.

a much longer lag than we measure, however). Upon inspection, we find that there are likely issues with the $\sigma_{*}$ measurements, all of which are below $100 \mathrm{~km} \mathrm{~s}^{-1}$ and approach the limits of the data used to measure them. We examined the spectral decomposition fits used to measure $\sigma_{*}$ in these four sources and found that, using the $\mathrm{Ca} \mathrm{H} / \mathrm{K}$ lines only, the measurements for these sources are significantly higher, indicating that the original measurements are likely

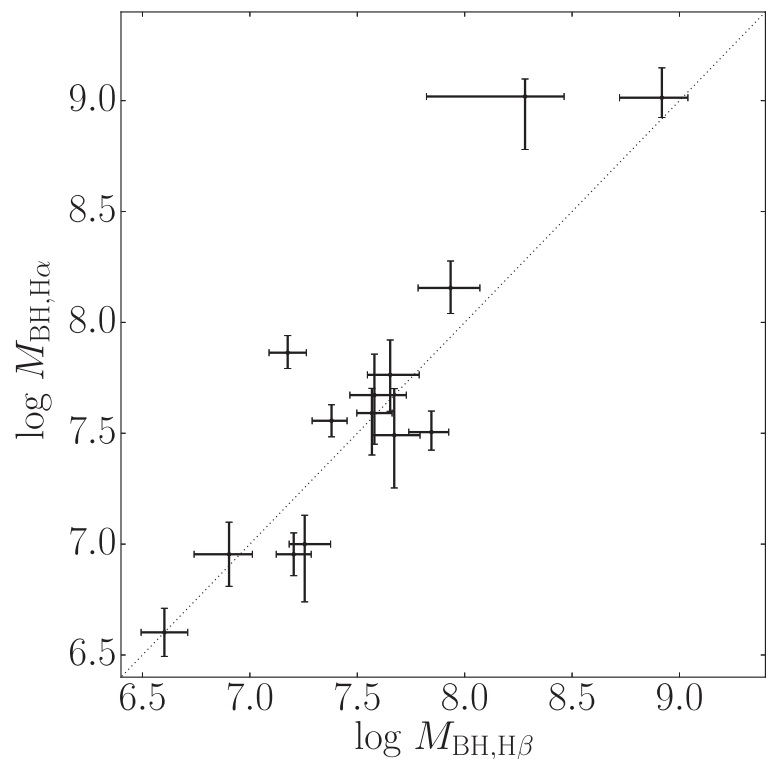

Figure 13. Comparison of $M_{\mathrm{BH}}$ measured from $\mathrm{H} \beta$ and $\mathrm{H} \alpha$ for those objects where we detected lags in both emission lines. The black dotted line shows a ratio of one-to-one.

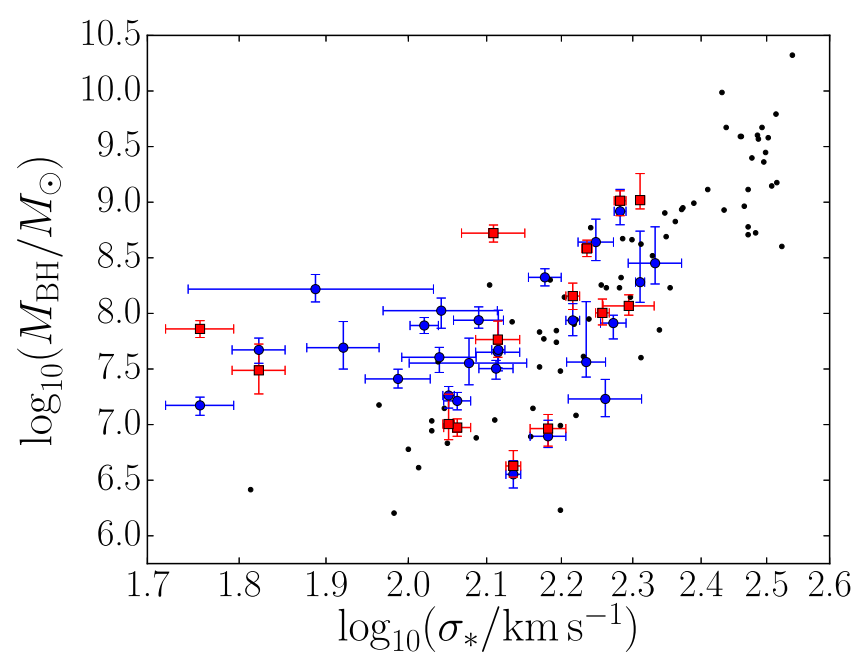

Figure 14. The $M_{\mathrm{BH}}-\sigma_{*}$ relation with the sample of dynamical black hole masses from McConnell \& Ma (2013) shown as black dots. Our new $M_{\mathrm{BH}}$ measurements made using the $\mathrm{H} \beta$ and $\mathrm{H} \alpha$ emission-line time lags and line widths are represented by blue circles and red squares, respectively.

underestimated; this is what causes them to appear as outliers on the $M_{\mathrm{BH}}-\sigma_{*}$ relation.

\subsection{Additional Sources of Systematics}

When obtaining RM $R_{\mathrm{BLR}}$ measurements, RM studies often make the assumption that the time delay between the $5100 \AA$ continuum-emitting region and a relevant broad emission line (such as $\mathrm{H} \beta$ ) is a good characterization of the distance between the $\mathrm{BH}$ and the $\mathrm{H} \beta$-emitting BLR. However, the $R_{\mathrm{BLR}}$ measured with RM is actually the distance between the optical continuum-emitting region and the BLR and not between the BLR and the BH itself; past RM efforts have generally assumed that the distance between the $\mathrm{BH}$ and the continuumemitting region is negligible. Recent work has indicated that the optical continuum-emitting region can have a significant lag relative to the ultraviolet (Collier et al. 1998; Sergeev 
et al. 2005; McHardy et al. 2014; Shappee et al. 2014; Edelson et al. 2015; Fausnaugh et al. 2016). Specifically, Fausnaugh et al. (2016) found that the $V$-band emitting region of NGC 5548 is 2 light-days farther out than the $1367 \AA$ UVemitting region in NGC 5548, a distance that is nonnegligible compared to the measured time lags for some of the broad emission lines (the He II $\lambda 1640$ emission line was measured to have a time delay of 2.4 days, for example). It is thus likely that our measurements of $R_{\mathrm{BLR}}$ using optical/BLR lags are underestimated.

Assuming a universal AGN accretion disk, where the distance between the UV and optical continuum-emitting regions is constant for all sources, a nonzero UV-optical time delay will not have an effect on $\mathrm{RM} M_{\mathrm{BH}}$ measurements because the scale factor $f$ automatically corrects, at least in a statistical sense, for this distance effect by requiring that AGNs fall on the quiescent $M_{\mathrm{BH}}-\sigma_{*}$ relation. However, if the UVoptical distance depends on $L$ or $M_{\mathrm{BH}}$, this complication could pose a problem. Pei et al. (2017) examine possible dependencies on quasar parameters such as $L$ and $M_{\mathrm{BH}}$ and report that the scaling with luminosity is expected to be slow. Microlensing studies also show that the size of the BLR more or less scales as expected with $M_{\mathrm{BH}}$ (Morgan et al. 2010; Mosquera et al. 2013).

In addition, the scatter in the $R-L$ relationship (Bentz et al. 2013 ) is small, so these effects are likely small for most AGNs, as a large UV-optical distance scaling would cause larger scatter in this relation. Thus far, there are only a few solid measurements of the UV-optical continuum time delay, so we are unable to directly measure any dependencies of UV-optical size with quasar properties. Additional measurements of interband continuum lags will be necessary to determine what (if any) correction is needed to account for the use of optical continua in measuring broad emission-line lags.

\section{Summary}

We have combined the spectroscopic and photometric observations from the first year of monitoring of the SDSSRM program to search for significant time delays in 222 quasars. Our major findings are the following.

1. We have measured characteristic time delays between the continuum and the $\mathrm{H} \beta$ and $\mathrm{H} \alpha$ broad emission lines in 44 and 18 sources, respectively. These measurements increase the size of the sample of AGNs that have reverberation mapping $M_{\mathrm{BH}}$ measurements by about twothirds. In addition, most of these measurements are made for higher-redshift objects, significantly expanding the redshift coverage of the RM sample. See Section 4.

2. We compared three different methods of obtaining lag measurements: the ICCF, JAVELIN, and CREAM. All three methods are generally consistent with one another, though JAVELIN (32 $\mathrm{H} \beta$ and $13 \mathrm{H} \alpha$ lags) and CREAM (42 $\mathrm{H} \beta$ and $17 \mathrm{H} \alpha$ lags) typical yield smaller uncertainties and thus more high-significance detections than the ICCF (16 $\mathrm{H} \beta$ and eight $\mathrm{H} \alpha$ lags). See Section 3.4.

3. We find that $\mathrm{H} \alpha$ lags are generally consistent with or larger than the $\mathrm{H} \beta$ lags measured in the same sources, which is consistent with previous findings. See Section 4.1.

4. We find that many of our sources fall below the $R-L$ relation measured by Bentz et al. (2013). This could be due to selection effects or a dependency of the $R-L$ relation on accretion parameters such as the Eddington ratio. See Section 4.1.

5. We measure $M_{\mathrm{BH}}$ for those objects with successful lag detections. Most of our measurements are consistent with the $M_{\mathrm{BH}}-\sigma_{*}$ relation measured in local quiescent galaxies, though we have some outliers at the low- $\sigma_{*}$ end of the relation that are likely due to selection effects or issues with $\sigma_{*}$ measurements. See Section 4.2.

With only the first year of data, we are sensitive only to lag measurements shorter than approximately 100 days in the observed frame. The next step is to incorporate the additional years of data from the SDSS-RM program to extend the lag sensitivity and the dynamic range in quasar luminosity. This will allow us to measure longer time delays and also will help remove aliases in our posterior lag distributions for shorter lags, which will likely reduce our false-positive rate. With the additional years of data that are already in hand or have been planned, we will also be able to investigate emission lines such as $\mathrm{C}$ IV and $\mathrm{Mg}$ II in higher-redshift targets, allowing us to probe quasars even farther out in the universe.

C.J.G., W.N.B., J.R.T., and D.P.S. acknowledge support from NSF grant AST-1517113. Y.S. acknowledges support from an Alfred P. Sloan Research Fellowship and NSF grant AST-1715579. C.S.K. is supported by NSF grant AST1515427. K.H. acknowledges support from STFC grant ST/ M001296/1. I.D.M. acknowledges support from NSF grant AST 15-15115. K.D.D. acknowledges support from the NSF awarded under NSF Grant AST-1302093. L.C.H. was supported by the National Key R\&D Program of China (2016YFA0400702) and the National Science Foundation of China (11473002, 11721303).

This work is based on observations obtained with MegaPrime/MegaCam, a joint project of CFHT and CEA/DAPNIA, at the Canada-France-Hawaii Telescope (CFHT), which is operated by the National Research Council (NRC) of Canada, the Institut National des Sciences de l'Univers of the Centre National de la Recherche Scientifique of France, and the University of Hawaii. The authors wish to recognize and acknowledge the very significant cultural role and reverence that the summit of Maunakea has always had within the indigenous Hawaiian community. The astronomical community is most fortunate to have the opportunity to conduct observations from this mountain.

Funding for SDSS-III has been provided by the Alfred P. Sloan Foundation, the Participating Institutions, the National Science Foundation, and the U.S. Department of Energy Office of Science. The SDSS-III website is http://www.sdss3.org/.

SDSS-III is managed by the Astrophysical Research Consortium for the Participating Institutions of the SDSS-III Collaboration including the University of Arizona, the Brazilian Participation Group, Brookhaven National Laboratory, Carnegie Mellon University, University of Florida, the French Participation Group, the German Participation Group, Harvard University, the Instituto de Astrofisica de Canarias, the Michigan State/Notre Dame/JINA Participation Group, Johns Hopkins University, Lawrence Berkeley National Laboratory, Max Planck Institute for Astrophysics, Max Planck Institute for Extraterrestrial Physics, New Mexico State University, New York University, Ohio State University, Pennsylvania State University, University of Portsmouth, Princeton University, the Spanish Participation Group, University of Tokyo, University 
of Utah, Vanderbilt University, University of Virginia, University of Washington, and Yale University.

We thank the Bok and CFHT Canadian, Chinese, and French TACs for their support. This research uses data obtained through the Telescope Access Program (TAP), which is funded by the National Astronomical Observatories, the Chinese Academy of Sciences, and the Special Fund for Astronomy from the Ministry of Finance in China.

\section{ORCID iDs}

C. J. Grier 낭 https://orcid.org/0000-0001-9920-6057

J. R. Trump (1) https://orcid.org/0000-0002-1410-0470 Yue Shen (ㄷ) https://orcid.org/0000-0003-1659-7035 Keith Horne (1) https://orcid.org/0000-0003-1728-0304 Karen Kinemuchi (ib https://orcid.org/0000-0001-7908-7724 Ian D. McGreer (ib https://orcid.org/0000-0002-3461-5228 W. N. Brandt (1) https://orcid.org/0000-0002-0167-2453 P. B. Hall $\odot$ https://orcid.org/0000-0002-1763-5825 C. S. Kochanek (1) https://orcid.org/0000-0001-6017-2961 L. C. Ho (1) https://orcid.org/0000-0001-6947-5846 B. M. Peterson (1) https://orcid.org/0000-0001-6481-5397 Mouyuan Sun (1) https://orcid.org/0000-0002-0771-2153 Dmitry Bizyaev (10) https://orcid.org/0000-0002-3601-133X Joel R. Brownstein (1) https://orcid.org/0000-0002-8725-1069 Kevin Bundy (i) https://orcid.org/0000-0001-9742-3138 K S. Dawson (1) https://orcid.org/0000-0002-0553-3805 Yang Gao (1) https://orcid.org/0000-0002-6316-1632 Timothy A. Hutchinson (ib https://orcid.org/0000-00023009-260X

Linhua Jiang (i) https://orcid.org/0000-0003-4176-6486 Kaike Pan (1) https://orcid.org/0000-0002-2835-2556 Kara A. Ponder (1) https://orcid.org/0000-0002-8207-3304 Jesse Rogerson (i) https://orcid.org/0000-0002-7078-1776 Audrey Simmons (i) https://orcid.org/0000-0002-2364-7240

\section{References}

Ahn, C. P., Alexandroff, R., Allende Prieto, C., et al. 2014, ApJS, 211, 17 Alard, C. 2000, A\&AS, 144, 363

Alard, C., \& Lupton, R. H. 1998, ApJ, 503, 325

Alexander, T. 1997, Astrophysics and Space Science Library, 218, 163

Almaini, O., Lawrence, A., Shanks, T., et al. 2000, MNRAS, 315, 325 Aune, S., Boulade, O., Charlot, X., et al. 2003, Proc. SPIE, 4841, 513 Barth, A. J., Bennert, V. N., Canalizo, G., et al. 2015, ApJS, 217, 26 Bentz, M. C., Denney, K. D., Grier, C. J., et al. 2013, ApJ, 767, 149 Bentz, M. C., \& Katz, S. 2015, PASP, 127, 67

Bentz, M. C., Walsh, J. L., Barth, A. J., et al. 2009, ApJ, 705, 199 Bentz, M. C., Walsh, J. L., Barth, A. J., et al. 2010, ApJ, 716, 993 Blandford, R. D., \& McKee, C. F. 1982, ApJ, 255, 419 Blanton, M. R., Bershady, M. A., Abolfathi, B., et al. 2017, AJ, 154, 28 Cameron, E. 2011, PASA, 28, 128

Collier, S. J., Horne, K., Kaspi, S., et al. 1998, ApJ, 500, 162

Dawson, K. S., Schlegel, D. J., Ahn, C. P., et al. 2013, AJ, 145, 10 Denney, K. D., Horne, K., Shen, Y., et al. 2016, ApJS, 224, 14 Denney, K. D., Peterson, B. M., Pogge, R. W., et al. 2010, ApJ, 721, 715 Doi, M., Tanaka, M., Fukugita, M., et al. 2010, AJ, 139, 1628
Du, P., Hu, C., Lu, K.-X., et al. 2014, ApJ, 782, 45

Du, P., Hu, C., Lu, K.-X., et al. 2015, ApJ, 806, 22

Du, P., Lu, K.-X., Hu, C., et al. 2016a, ApJ, 820, 27

Du, P., Lu, K.-X., Zhang, Z.-X., et al. 2016b, ApJ, 825, 126

Edelson, R., Gelbord, J. M., Horne, K., et al. 2015, ApJ, 806, 129

Edelson, R. A., \& Krolik, J. H. 1988, ApJ, 333, 646

Eisenstein, D. J., Weinberg, D. H., Agol, E., et al. 2011, AJ, 142, 72

Fausnaugh, M. M., Denney, K. D., Barth, A. J., et al. 2016, ApJ, 821, 56

Fausnaugh, M. M., Grier, C. J., Bentz, M. C., et al. 2017, ApJ, 840, 97

Fukugita, M., Ichikawa, T., Gunn, J. E., et al. 1996, AJ, 111, 1748

Gaskell, C. M., \& Peterson, B. M. 1987, ApJS, 65, 1

Goad, M. R., Korista, K. T., De Rosa, G., et al. 2016, ApJ, 824, 11

Grier, C. J., Hall, P. B., Brandt, W. N., et al. 2015, ApJ, 806, 111

Grier, C. J., Peterson, B. M., Pogge, R. W., et al. 2012, ApJ, 755, 60

Gunn, J. E., Siegmund, W. A., Mannery, E. J., et al. 2006, AJ, 131, 2332

Hartman, J. D., Bakos, G., Stanek, K. Z., \& Noyes, R. W. 2004, AJ, 128, 1761

Hu, C., Du, P., Lu, K.-X., et al. 2015, ApJ, 804, 138

Jiang, Y.-F., Green, P. J., Greene, J. E., et al. 2017, ApJ, 836, 186

Kaspi, S., Brandt, W. N., Maoz, D., et al. 2007, ApJ, 659, 997

Kaspi, S., Maoz, D., Netzer, H., et al. 2005, ApJ, 629, 61

Kaspi, S., Smith, P. S., Netzer, H., et al. 2000, ApJ, 533, 631

Kelly, B. C., Bechtold, J., \& Siemiginowska, A. 2009, ApJ, 698, 895

Korista, K. T., \& Goad, M. R. 2004, ApJ, 606, 749

Kozłowski, S. 2016, MNRAS, 459, 2787

Kozłowski, S. 2017, A\&A, 597, A128

Kozłowski, S., Kochanek, C. S., Udalski, A., et al. 2010, ApJ, 708, 927

Li, J., Shen, Y., Horne, K., et al. 2017, ApJ, 846, 79L

MacLeod, C. L., Ivezić, Ž., Kochanek, C. S., et al. 2010, ApJ, 721, 1014

MacLeod, C. L., Ivezić, Ž., Sesar, B., et al. 2012, ApJ, 753, 106

Maronna, R. A., Martin, R. D., \& Yohai, V. J. 2006, Robust Statistics (New York: Wiley)

Matsuoka, Y., Strauss, M. A., Shen, Y., et al. 2015, ApJ, 811, 91

McConnell, N. J., \& Ma, C.-P. 2013, ApJ, 764, 184

McHardy, I. M., Cameron, D. T., Dwelly, T., et al. 2014, MNRAS, 444, 1469

Metzroth, K. G., Onken, C. A., \& Peterson, B. M. 2006, ApJ, 647, 901

Morgan, C. W., Kochanek, C. S., Morgan, N. D., \& Falco, E. E. 2010, ApJ, 712, 1129

Mosquera, A. M., Kochanek, C. S., Chen, B., et al. 2013, ApJ, 769, 53

Netzer, H. 1975, MNRAS, 171, 395

Onken, C. A., Ferrarese, L., Merritt, D., et al. 2004, ApJ, 615, 645

Pei, L., Fausnaugh, M. M., Barth, A. J., et al. 2017, ApJ, 837, 131

Peterson, B. M. 2011, in Narrow-Line Seyfert 1 Galaxies and their Place in the Universe, ed. L. Foschini et al. (Trieste: SISSA), 034

Peterson, B. M., Ferrarese, L., Gilbert, K. M., et al. 2004, ApJ, 613, 682

Rees, M. J., Netzer, H., \& Ferland, G. J. 1989, ApJ, 347, 640

Sergeev, S. G., Doroshenko, V. T., Golubinskiy, Y. V., Merkulova, N. I., \& Sergeeva, E. A. 2005, ApJ, 622, 129

Shappee, B. J., Prieto, J. L., Grupe, D., et al. 2014, ApJ, 788, 48

Shen, Y., Brandt, W. N., Dawson, K. S., et al. 2015a, ApJS, 216, 4

Shen, Y., Brandt, W. N., Richards, G. T., et al. 2016a, ApJ, 831, 7

Shen, Y., Greene, J. E., Ho, L. C., et al. 2015b, ApJ, 805, 96

Shen, Y., Horne, K., Grier, C. J., et al. 2016b, ApJ, 818, 30

Shen, Y., Richards, G. T., Strauss, M. A., et al. 2011, ApJS, 194, 45

Smee, S. A., Gunn, J. E., Uomoto, A., et al. 2013, AJ, 146, 32

Starkey, D., Horne, K., Fausnaugh, M. M., et al. 2017, ApJ, 835, 65

Starkey, D. A., Horne, K., \& Villforth, C. 2016, MNRAS, 456, 1960

Sun, M., Trump, J. R., Shen, Y., et al. 2015, ApJ, 811, 42

Vestergaard, M., \& Peterson, B. M. 2006, ApJ, 641, 689

White, R. J., \& Peterson, B. M. 1994, PASP, 106, 879

Williams, G. G., Olszewski, E., Lesser, M. P., \& Burge, J. H. 2004, Proc. SPIE, 5492,787

Woo, J.-H., Yoon, Y., Park, S., Park, D., \& Kim, S. C. 2015, ApJ, 801, 38

Zu, Y., Kochanek, C. S., Kozłowski, S., \& Udalski, A. 2013, ApJ, 765, 106

Zu, Y., Kochanek, C. S., \& Peterson, B. M. 2011, ApJ, 735, 80 